\title{
ABSTRACTS/RÉSUMÉS
}

\section{Canadian Society of Plastic Surgeons Société Canadienne des Chirurgiens Plasticiens}

\author{
Abstracts presented at the \\ 64th Annual Meeting / 64 ${ }^{\mathrm{e}}$ Réunion annuelle \\ June 15-19, 2010, Halifax, Nova Scotia
}

Dr Justin Paletz: President / Président

Dr David D Jewer: Vice-President / Vice-Président

Chair, Scientific Program / Président, Programme scientifique

Dr Steven Morris: Chair, Local Organizing Committee / Président, Intendance générale

\section{Eye-Opener Session}

\section{REJUVENATION AND RECONSTRUCTION WITH STRUCTURAL FAT}

\section{Sydney R Coleman, MD}

Atrophy plays an extremely important role in facial aging. The current model of aging is based upon loss of volume in the face as the primary physical sign of aging followed or accompanied by sagging or descent of soft tissues. Restoration of fullness to the atrophic, aging upper and midface is essential for successful rejuvenation. Adding fullness to the face is a natural, logical approach to rejuvenation.

In the early 1990's, I first noted that transplanted fat did more than just create fullness. The implanted fat improved the quality of the tissues into which it was grafted. This initial improvement is then usually followed by a continued, gradual long-term improvement in the quality of the skin. Wrinkles soften, pore size decreases and pigmentation improves in patients followed over ten years. In addition, fat grafted under depressed scars not only relieves the depression, but also softens or even completely eliminates the specific scar tissue, making it look like normal skin. More recently we have noted the effect of fat grafts on therapeutic radiation injuries.

But grafted fat does more than simply restore fullness. Changes occur in skin overlying the grafted sites over time (for instance, immediately after implantation, in the first months, at one year, three years, five years, eight years etc). The volume of the fat appears to stabilize four months after the procedure, but the texture of sun damaged or scarred skin continues to improve dramatically in the ensuing months. The improvement usually continues up to five years and in many cases for almost a decade.

While fat grafting was used mainly in aesthetic surgery for many years, we are now applying these same techniques for reconstructive surgery to restore or create facial or corporal forms by manipulation of soft tissues. Examples of facial and body reconstruction will be presented.

Structural fat grafting can be a relatively predictable and safe procedure with a low rate of complications and a high degree of patient satisfaction. Further studies are essential to understand more clearly the mechanism of grafted fat tissue and increase predictability.

Learning Objectives:

- To better appreciate the potential of structural fat grafting in facial rejuvenation.

- To identify the potential effect of fat grafts on scarring.

- To understand the potential uses in facial and corporal reconstruction.

- To introduce recent advances in the use of fat grafting.

Disclosure of Interest:

Dr. Coleman has noted the following:

1) I have the potential for stock options with Cytori Therapeutics and Brava.

2) I do research into techniques using products from Mentor.

3) I receive royalties and consulting fees from Mentor.

\section{General Session}

\section{1}

AN AUDIT OF PRIMARY PALATOPLASTY; RESULTS FROM ONE SURGEON AND 485 CASES OVER 10 YEARS

MH Mahoney (presented by D Fisher)

PURPOSE: The purpose of this study is the review of a single surgeon's protocol and experience in primary palatoplasty over a 10 year period at a single cleft centre.
METHODS: A retrospective review of a single surgeon's prospectively acquired database was undertaken. The study describes: 1) the demographics of the patient population, 2) the distribution of cleft palate type (Veau and Kernahan \& Stark classifications) and cleft severity (palate length and cleft width), and 3) early (fistula rate) and mid-term (rate of secondary surgery for VPI) results.

RESULTS: 524 palatoplasties were performed from January 1, 2000 through January 1, 2010. Of these cases, 485 primary palatoplasties were performed. The mean age was 20.4 months (range 6.6 months 17.7 years). There were 276 males and 209 females. Clefts were classified according to Kernahan and Stark $(\mathrm{CP}=260, \mathrm{CL} / \mathrm{P}=225)$ and Veau Class (I=85, II-175, III-165, IV-60). Palate length was assessed according to Randall's classification $(I=81, I I=319, I I I=58, I V=2)$. Palate width, measured on the day of surgery, averaged $7.7 \mathrm{~mm}$ (range $0-19 \mathrm{~mm}$ ). The surgical technique used was dictated by cleft type (Veau classification); 78 Furlow, 101 Veau, 191 von Langenbeck, 114 Clarke, and 1 other. There were 4 fistulas $(0.8 \%)$. To date 50 patients have undergone secondary procedures for VPI (22 secondary Furlow palatoplasties and 28 pharyngeal flap pharyngoplasties). Over this 10 year period, the protocol has been consistent, with two exceptions to be discussed.

CONCLUSIONS: Demographics, procedures, and results of 485 consecutive palatoplasties are reviewed. The fistula rate is less than 1 percent. The intermediate VPI rate is at least 10 percent.

Learning Objectives:

After viewing this presentation, the learner will:

- Be familiar with the demographics of palatal clefts.

- Have reviewed one surgeon's protocol for and results of primary palatoplasty.

- Be familiarized with current trends in cleft palate repair.

\section{2}

\section{THE OUTCOME OF PRIMARY PALATE CLOSURE AT THE SAME TIME AS CLEFT LIP REPAIR}

\section{$\underline{L}$ Bergeron, L Caouette-Laberge, N Ouatik, H El-Khatib}

PURPOSE: Closure of the primary palate with a vomer flap at the time of cleft lip repair is controversial. It is often suggested that it should be delayed since additional scarring could negatively affect maxillary growth. Our center has routinely closed the primary palate at the time of lip repair since 1963 and no additional growth disturbance has been noted. The purpose of this study is to compare sagittal maxillary growth of patients operated in our center to published series of cleft and normal children.

METHOD: Patients with complete unilateral cleft lip and palate operated between 1983 and 1989 were included. SNA and ANB cephalometric values at 9 and 17 years old were compared to well documented series from other centers and a normal reference group. Statistical analysis was conducted to determine if our group was equal or better than reported results.

RESULTS: 34 patients were identified. Maxillary growth, documented with SNA and ANB angles, was better than reported in other large series of cleft children $(\mathrm{p}<0.05$, one tailed $\mathrm{t}$-test $)$. As in other series, the values obtained for our cleft patients were inferior to the normal population except for ANB measurements at 9 years old, which were similar. 
CONCLUSION: Sagittal maxillary growth is not worsened by primary palate closure with a vomer flap at the time of lip repair. It does not produce additional maxillary deformity. It is a safe procedure that offers numerous advantages linked to the prevention of residual oronasal fistulas.

Learning Objectives:

At the end of this presentation, the participant will:

- Understand the controversy surrounding early primary palate closure.

- Understand the benefits of closing the primary palate at the time of cleft lip repair.

\section{3}

\section{MODIFIED LATISSIMUS DORSI PEDICLED MUSCLE TRANSFER TO RESTORE EXTERNAL ROTATION IN BRACHIAL PLEXOPATHY: A BIOMECHANIC STUDY AND CASE REPORT}

P Tétreault, DM Tremblay, A Chollet, $\underline{\mathrm{LC} \text { Lin }}$

PURPOSE: Loss of external rotation due to brachial plexus injury is functionally debilitating and few secondary procedures exist to correct this deficiency. The Latissimus dorsi has been used as a tendon transfer to restore external rotation in rotator cuff tear patients. In some reports, however, patients with this transfer do not regain active external rotation past $0^{\circ}$, and experience no increase in force. We hypothesize that this is due to the biomechanically disadvantaged line of pull of the latissimus dorsi muscle.

METHODS: Using a cadaveric biomechanical model with the arm at $0^{\circ}$ of abduction, we determined the forces necessary to go from $0^{\circ}$ to $40^{\circ}$ of external rotation using two different lines of pull. The first was in the natural line of pull of the latissimus dorsi muscle, with the insertion transferred to the greater tuberosity of the humerus, and the second was in the natural line of pull of the infraspinatus muscle, with the same insertion site.

RESULTS: On average, $40 \%$ greater force was necessary to move the cadaveric upper limb into external rotation using the original line of pull of the latissimus muscle. Reorienting the line of pull to the axis of the infraspinatus allowed more degrees of external rotation with the application of less force. One brachial plexus patient with no active external rotation consented to a modified latissimus dorsi muscle transfer reorienting the muscle's line of pull to that of the infraspinatus muscle. Clinical results will be presented.

CONCLUSION: This biomechanic cadaveric study showed that reorienting the latissimus dorsi muscle's line of pull into that of the infraspinatus muscle decreases the force necessary for external rotation, suggesting that clinical results can be improved with this muscle transfer by modifying the origin and insertion of the muscle.

Learning Objectives:

- Participants will learn a new technique for restoring external rotation.

\section{4}

\section{Canadian Expert Series}

\section{TREATMENT OF STERNAL INFECTION: CONTINUING EVOLUTION}

\section{JL Mahoney}

Open-heart surgery is one of the most common procedures performed in North America. The median sternotomy has provided the access for various heart procedures. Infection and dehiscence are two commonly described complications which occur in $1-3 \%$ of these patients. These complications are associated with significant morbidity and mortality. Timely surgical intervention is required. Debridement of the sternum is critical in removing the infected tissue and bone. Reconstruction of the chest wall has evolved with a variety of closure techniques. Sternal rewiring and closure, a variety of flaps, and more recently adjunctive negative pressure therapy have been recommended as options. New techniques of rigid fixation of the sternum have been introduced. Their potential advantage is the restoration of sternal stability following sternal debridement which has not previously been feasible with flaps or rewiring. We have had our greatest success with the rigid fixation technique and find that most patients can be salvaged following wound and sternal dehiscence with debridement and sternal plating in one stage. Our evolving approach to this potentially increasingly frequent complication will be reviewed.

Learning Objectives:

- Diagnosis of sternal complications after cardiac surgery.

- Review the principles of treatment.

- Discuss the management of the sternal wound following debridement.

- Discuss the surgical outcomes.

\section{5}

\section{FREE FLAP RECONSTRUCTION IN MANAGEMENT OF LOCALLY RECURRENT SOFT TISSUE SARCOMA AFTER FAILURE OF MULTIMODALITY TREATMENT}

SOP Hofer, MA Al-Yamani, AA Van Driel, A Griffin, BM Deheshi, PC Ferguson, JS Wunder

PURPOSE: To investigate the outcome of free flap (FF) reconstruction in limb salvage of locally recurrent extremity soft tissue sarcoma (STS) following prior surgery and radiation.

METHODS: Patients were identified from a prospective database. Individual charts were reviewed for clinicopathologic characteristics, reconstruction technique, complications, oncologic outcome, and limb function by Musculoskeletal Tumor Society Score (MSTS) and Toronto Extremity Salvage Score (TESS) compared with results following amputation (AMP).

RESULTS: Seventeen patients underwent FF reconstruction and 32 had AMP in management of locally recurrent STS after failure of prior multimodality treatment. There were no differences in clinicopathologic features between the two groups. Wound related complications were more common following FF reconstructions $(9 / 17,52 \%)$ than after AMP $(4 / 32,13 \% ; p=0.08)$, as was the risk of additional surgery $(4 / 17$ vs. $1 / 32$; $\mathrm{p}=0.043)$. However, the mean functional scores at 1 year follow-up were MSTS87=28.8/35, MSTS93 $=81.6$ and TESS=78.3. Local tumor relapse occurred in 4/17 (24\%) FF patients necessitating 1 amputation, compared to $6 / 32(19 \%)$ AMP patients leading to 4 additional procedures. There was no difference in 5-year disease-free survival and overall survival between FF and AMP groups ( $\mathrm{p}=0.2113)$.

CONCLUSIONS: Free flap reconstruction in the management of recurrent sarcoma following prior radiation is a complicated procedure associated with a higher than usual risk of treatment-related complications including flap failure. However, limb salvage in this setting is associated with functional outcome scores comparable to patients undergoing primary sarcoma management.

Learning Objectives:

- Free flap reconstruction following multimodal cancer failure is a practical option associated with functional outcome scores comparable to patients undergoing primary sarcoma management.

\section{6}

\section{CONTRALATERAL PROPHYLACTIC MASTECTOMY}

SS Rai, RC Mahabir, JW Roberts, J Song, KS Hamid, RR White IV PURPOSE: To identify the prevalence of pathology within the specimen at the time of contralateral prophylactic mastectomy (CPM) and to evaluate potential risk factors.

METHODS: Patients with a unilateral breast malignancy who underwent CPM between January 2004 and July 2009 were identified from the Scott and White Breast Cancer database. Inclusion criteria for a $\mathrm{CPM}$ was a clinically and radiologically negative breast examination. A retrospective cohort study comparing disease status and various exposure parameters was conducted via chart review.

RESULTS: Of the 1,011 mastectomies evaluated, 301 CPMs met the inclusion criteria. Of these patients, there were 14 cases $(4.7 \%)$ with malignancy and 45 cases (15.0\%) with moderate-to-high risk lesions. The combined prevalence of malignancy and moderate-to-high risk lesions was $19.7 \%$. Multivariate analysis demonstrated two independent factors predictive of malignant / moderate-to-high-risk lesions: age greater than 54 and lobular history in the original specimen. 
CONCLUSIONS: There is a significant prevalence of clinically undetectable malignant and pre-malignant lesions at the time of contralateral prophylactic mastectomy.

Learning Objective:

- At the end of this lecture, the participants will be able to report the rate of malignant and pre-malignant lesions in a contralateral prophylactic mastectomy specimen.

\section{7}

\section{OUTCOMES AND COMPLICATIONS USING A NOVEL TECHNIQUE OF FRONTAL-ORBITAL REMODELING FOR NON-SYNDROMIC CORONAL AND METOPIC CRANIOSYNOSTOSIS}

\section{SKF Seal, DJ Courtemanche, P Steinbok}

PURPOSE: The purpose of this study is to review the results and complications associated with the $\mathrm{BCCH}$ technique of frontal-orbital remodeling for metopic and coronal synostoses. Absorbable plate fixation has become a common method in frontal-orbital fixation. Our study uses 2-0 Vicryl to hold the advancement in place. This is the first comprehensive report of outcomes using this technique in the literature.

METHOD: A retrospective chart review was conducted on patients with non-syndromic metopic and coronal craniosynostoses admitted for frontal-orbital surgery at the BCCH in Vancouver from 1985 to 2009. RESULTS: A total of 106 patients were reviewed from September 1985 to June 2009. The mean operative time was $183+/-41$ minutes. Blood loss mean was $150+/-126 \mathrm{cc}$. The number of patients requiring blood transfusions was 26. Mean transfusion of packed red cells was $207+/-102$ cc.

Complications during the operation are as follows: dural tears in 13 patients, subdural hematoma in 1 patient, and nasal greenstick fracture in 1 patient. No patients returned to the $\mathrm{OR}$ in the peri-operative period. Length of stay in hospital was 3.7 days (SD 1.2).

Total re-operation rate was 6 patients, for contour irregularities (5), and for a widened scalp scar (1).

CONCLUSIONS: This paper is the first report in the literature to describe this technique for frontal-orbital advancement. In our opinion, this technique is less complicated, time efficient, and less resource intensive than other techniques reported in the literature. Furthermore, the low re-operation rate indicates an acceptable esthetic outcome.

Learning Objectives:

- Participants will become familiar with various techniques of fronto-orbital advancement.

- Participants will learn of a different technique for fronto-orbital advancement used at BCCH.

- Participants will become familiar with the complications and outcomes using this fronto-orbital advancement technique.

\section{8}

\section{ACQUISITION OF EVIDENCE-BASED SURGERY SKILLS IN PLASTIC SURGERY RESIDENCY TRAINING}

\section{Temple, DC Ross}

INTRODUCTION: Teaching critical appraisal skills is mandated by the Royal College but remains a difficult body of knowledge to impart to surgical residents.

METHODS: Monthly journal clubs over a calendar year were the setting for a critical appraisal curriculum. Pre-assigned homework and carefully selected articles with specific methodologies were posted electronically and formed the course material. Pre- and post-tests on medical statistics and methodology were administered. Pre- and postsurveys on attitudes toward Evidence-Based Surgery (EBS) were administered.

RESULTS: Pre-course surveys revealed a lack of confidence in residents' knowledge of Epidemiology and Biostatistics, with a non-significant increase in confidence post-course ( 2.6 vs. $2.9 \mathrm{p}=0.4)$. Pre- and postcourse, there was strong agreement to include more critical appraisal training in residency ( 5.1 vs. $4.8 \mathrm{p}=0.10$ ), and that understanding EBS is important for the clinical practice $(4.6$ vs. $4.6, p=0.4)$ and the research endeavors of a plastic surgeon (5.4 vs. $5.5, \mathrm{p}=0.8$ ).

Pre-test scores, as a function of training level, showed a non-significant $(p=0.62)$ increase in knowledge with increasing PGY level. Average pre-test scores were 6.5 out of a total of 15 points, or $43 \%$. Post-test scores were improved, at 7.8 out of 15 , or $52 \%$. $(\mathrm{p}=0.59)$. Sixtyfour percent of students felt that journal club was a good venue for teaching critical appraisal skills pre-curriculum, yet only $50 \%$ were still of that impression at the completion $(p=0.3)$. The modest improvement in test scores indicates that we can impact critical appraisal skills, but will require a long-term commitment and expanded settings and methods of teaching.

CONCLUSION: Through monthly journal clubs and self-directed assignments, critical appraisal skills were improved across PGY levels in an academic surgical training program. Adjunctive teaching methods are required to further enhance knowledge of EBS during residency training.

Learning Objective:

- To understand an approach to teaching critical appraisal skills to residents.

\section{9}

\section{POSTERIOR CRANIAL VAULT DISTRACTION IN THE MANAGEMENT OF SYNDROMIC CRANIOSYNOSTOSIS \\ C Forrest}

INTRODUCTION: Cranial vault reshaping for the purposes of expansion and normocephaly in syndromic craniosynostosis is associated with significant relapse, residual deformity (turribrachycephaly) and high rates of reoperation. The purpose of this study was to present the experience with posterior cranial vault distraction as a first stage in the management of syndromic craniosynostosis.

METHODS: 4 patients (2M, 2F: age 6 months to 61 months) with syndromic craniosynostosis (2 Aperts, $17 \mathrm{p}$-deletion, 1 unknown) seen at the Hospital for Sick Children presenting with severe turribrachycephaly underwent posterior craniotomy with application of KLSMartin distractors. A posterior coronal flap was elevated in a sub-galeal fashion. Periosteum was divided along the craniotomy line but the bone was left attached to dura. Distraction $(0.5 \mathrm{~mm}$ bid) commenced on the first post-operative day and the consolidation period was 8 to 10 weeks. Mean distraction distance was $20 \mathrm{~mm}$.

RESULTS: No mortalities or significant morbidities were encountered. Only 1 patient underwent transfusion (starting $\mathrm{Hb} 85$ ). All patients had satisfactory expansion of the posterior cranial vault with decreased turricephaly. A technical distractor failure resulted in a reoperation with successful outcome. Two patients subsequently underwent anterior cranial vault reshaping to achieve normocephaly. The two other patients have undergone significant spontaneous remodeling of the anterior frontal-orbital region.

CONCLUSION: Posterior cranial vault distraction provides a controlled expansion of the brachycephalic cranium with correction of turricephaly. Disadvantages of a second procedure to remove the distractors and cost of hardware may be outweighed by the advantages of shorter procedure time, less blood loss, no need to remove bone over the confluence of the venous sinuses and spontaneous remodeling of the forehead.

Learning Objective:

- To become familiar with the application of posterior cranial vault reshaping in the management of syndromic craniosynostosis.

\section{0}

\section{EVALUATION OF STRABISMUS IN PATIENTS OPERATED FOR ANTERIOR SYNOSTOTIC PLAGIOCEPHALY}

\section{A Dumas, $P$ Bortoluzzi}

OBJECTIVES: The presence of strabismus is well known among patients presenting with unilateral coronal craniosynostosis. There have been no studies in the literature that have quantifiably looked at the effect of reconstructive cranio-orbital surgery on strabismus in this patient population. The goal of this study is to quantifiably evaluate the impact that fronto-orbital remodeling has on strabismus. 
METHODS: We reviewed all patients affected with synostotic anterior plagiocephaly from 1987 to 2007 at Sainte Justine Hospital. Prospective ophthalmological evaluations included pre and post operative visual acuity, funduscopic assessment, as well as refractions and motility studies. Surgery was done at approximately 12 months of age, while initial and first post-operative ophthalmological assessments were done at 8.6 and 22.8 months respectively. All of the patient charts were systematically analyzed retrospectively with an ophthalmologist and the data were standardized. The Student t-test and the Pearson chi-square test were used for statistical analysis.

RESULTS: Ninety-eight patient charts were reviewed. Forty-nine patients filled the inclusion criteria $(35 \mathrm{~F}, 14 \mathrm{M})$. The right coronal suture was affected in 31 patients and the left in 18 .

The general incidence of strabismus pre-operatively was $57.1 \%(n=28)$. The ipsilateral eye to the synostosis demonstrated a vertical strabismus in 20 patients. The average objective value of the strabismus was 15.25 (6.61 SD) diopter prisms (DP). Moreover, 12 patients had horizontal strabismus of the ipsilateral eye with an average of 28.10 (18.16 SD) DP.

Post-operative incidence of strabismus was $63.3 \%(n=31)$. The eye on the synostotic side presented a vertical strabismus in 21 patients, with an average value of 11.68 (6.52 SD) DP. The horizontal strabismus of those eyes was present in 15 patients with an average value of 24.27 (16.32 SD) DP.

In general, the post-operative evolution of the strabismus of each individual patient was stable in $42.5 \%$ of the patients $(n=17)$, improved in $30 \%(n=12)$ of the patients, and deteriorated in $27.5 \%(n=11)$ of the patients.

When we statistically analyzed the pre and post operative differential for each patient individually, there was no statistically significant difference in diopter prisms of the vertical or horizontal strabismus.

CONCLUSION: This review represents the first quantifiable study in the literature of pre and postoperative strabismus in a large patient population with anterior synostotic plagiocephaly, which is the most common craniosynostosis requiring cranio-orbital reconstruction. The functional aspects of surgical correction are frequently questioned. This study demonstates that with regards to the strabismus that the majority of these patients present, the evolution following cranio-orbital surgery is unpredictable.

Learning Objectives:

- To understand the orbital anatomy in patients with anterior synostotic plagiocephaly.

- To understand the pathophysiology of the strabismus in these patients.

- To evaluate the impact of cranio-orbital surgery on this strabismus.

\section{1}

\section{PRELIMINARY RESULTS OF THE FIRST PERMANENT HUMAN IMPLANT OF THE STIMULUS ROUTER SYSTEM, A NOVEL NEURAL PROSTHESIS}

\section{LS Gan, A Prochazka, LOlson, M Morhart, EN Ravid, J Kowalczewski}

PURPOSE: Neural prostheses (NPs) are electrical stimulators that help to restore sensory or motor functions lost as a result of neural damage. We introduce the Stimulus Router System (SRS) a new system that is inexpensive, improves selectivity, and decreases surface discomfort.

METHODS: In June 2008, the SRS was implanted in a tetraplegic man with a C5/6 level spinal cord injury. Three SRS leads were implanted in his right forearm for activation of the finger extensors, finger flexors and thumb flexors for grasp and release. Previous acute and chronic animal studies have suggested that the SRS is safe and offers advantages that justify human trials.

RESULTS: Prior to surgery, activation thresholds for finger extensors and flexors with pulsatile stimuli delivered via conventional surface electrodes were $7.5 \mathrm{~mA}$ and $8.5 \mathrm{~mA}$ respectively and sensory threshold was $\sim 6 \mathrm{~mA}$. Maximal grip strength of $5 \mathrm{lbs}$ was elicited at a surface current of $19 \mathrm{~mA}$. At 10 days after surgery, the surface currents needed to activate the finger extensors and flexors via the SRS were $2.75 \mathrm{~mA}$ and $1.75 \mathrm{~mA}$ respectively. Maximal grip strength of $20 \mathrm{lbs}$ was elicited at a surface current of $3 \mathrm{~mA}$, about $50 \%$ of sensory threshold. The implants remain functional at the time of this report, 19 months after implantation. The subject continues to use the system daily to perform activities of daily life, switching between grasp and release with a wireless earpiece that detects toothclicks.

CONCLUSIONS: The results show that the SRS can selectively and functionally activate human muscles without causing discomfort or pain. It can produce stronger muscle contractions at lower surface currents than conventional surface stimulation. In principle, the SRS could be used for a variety of neuroprosthetic applications. We therefore believe it may form the basis of a new family of NPs.

Learning objectives

- The principles of neuroprosthesis.

- The previous downfalls associated with neuroprosthesis.

- The principles of direct targeted stimulation.

\section{2}

\section{TARGETED REINNERVATION FOR MYOELECTRIC PROSTHESIS}

\section{Morhart, J Olson, R Stiegelmar, J Hebert}

PURPOSE: Conventional body powered prosthesis and myoelectric prosthesis are limited in their usefulness. They allow only one degree of freedom with the result that an amputee can only control one movement at a time. Targeted reinnervation provides additional myoelectric control sites and therefore control of multiple movements at a time. It in essence acts as a direct wiring to the brain. We propose that targeted reinnervation for myoelectric prosthesis improves function for the patient.

METHODS: Three male patients with transhumeral amputations have had targeted reinnervation over a two year period. In these patients the median nerve is transferred to medial biceps, musculocutaneous nerve is left to the lateral biceps, the proximal radial nerve is left to the long head of triceps and the distal radial nerve is transferred to medial head of triceps. This gives four distinct areas of myoelectric signals. They have been fitted with their new prosthesis and trained in the use.

RESULTS: The follow-up ranges from 11- 23 months. Patients adapted to the targeted reinnervaton prosthesis much more quickly than to traditional body powered or myoelectric prosthesis. The new prosthesis improves dexterity and speed in performing tasks. With these advantages, wear times for the prosthesis are longer on a day to day basis.

CONCLUSION: Targeted reinnervation for myoelectric prosthesis is a new technology with only three having been performed in Canada and less than 50 worldwide. It provides the patient with much improved usability and control.

Learning Objectives:

- The audience will become familiar with prosthesis that are available to patients with amputations. The audience will further learn the pitfalls associated with traditional prosthesis.

\section{3}

\section{THE NATURAL HISTORY OF THE DUPUYTREN'S NODULE} C McMillan, YK Li, P Binhammer

PURPOSE: To examine the natural history of the untreated Dupuytren's nodule.

METHOD: Patients diagnosed with a Dupuytren's nodule between 1995 and 2005 were mailed a questionnaire evaluating the current characteristics of the nodule(s), symptoms of progression and changes in hand function. The Michigan Hand Questionnaire (MHQ) was administered to assess hand function.

RESULTS: One hundred, eighteen patients were identified. Twentyfive patients had surgery before study commencement and 38 patients either declined to participate, were deceased, or could not be reached due to outdated hospital records. Fifty-five patients completed the questionnaires (age, $67.4 \pm 9.7$ years). Mean follow-up from diagnosis to questionnaire completion was $8.2( \pm 2.9)$ years. Thirty-five respondents reported nodule progression, defined as an enlargement of the nodule since diagnosis and/or the presence of cords and/or permanent 
finger flexion. Ten respondents reported regression of the nodule and no cords or finger flexion. Nine respondents reported no change in nodule size since diagnosis and no cords or flexion, indicating nonprogression. Respondents reported that the nodule does not cause severe pain (mean, 1/5), severely affect their daily activities (mean, $1.5 / 5$ ), or the ability to straighten their fingers (mean, 2/5). Thirtyseven of the 55 respondents reported that they are able to place their hand flat on a tabletop. The mean MHQ score for the sample was $84.1 \pm 16.8$.

CONCLUSIONS: Of the patients who responded to the questionnaire, 10 reported nodule regression, 9 reported non-progression and 35 reported progression within 8.2 years.

Learning Objectives:

- To understand the natural history of a patient presenting with a Dupuytren's nodule.

\section{Tips \& Pearls}

\section{TP01}

CREATION OF AN "INTERNAL BRASSIERE": THE USE OF NON-RESORBABLE DERMAL SUSPENSION SUTURES TO ACHIEVE CONSISTENT, REPRODUCIBLE, AESTHETICALLY PLEASING BREAST MOUNDS

\section{K Boyd, N Guay}

Matching a contralateral ptotic breast to a unilateral implant-based reconstruction is challenging. We present our experience creating an "internal brassiere" using a non-resorbable dermal suture. This technique is ideal for mimicking the shape of an implant reconstruction, but also has utility in mastopexy, reduction mammoplasty, and massive weight loss reconstruction.

Learning Objectives:

- To understand the indications for creation of an "internal brassiere".

- To understand the surgical technique of placing a non-resorbable dermal suture to create internal support.

\section{TP02}

\section{HOW TO FIX A BRACHIAL PLEXUS}

H Clarke

This video presentation will demonstrate the complete reconstruction of the brachial plexus in an infant with obstetrical brachial plexus palsy. The preparation and placement of sural nerve grafts and the use of fibrin glue will be shown. The reconstruction reconstitutes the original plexus architecture.

\section{TP03}

\section{CAUDAL SEPTAL REPOSITIONING AS AN ADJUNCT TO PRIMARY CLEFT LIP REPAIR}

\section{Fisher}

Septal deviation is typical of the Cleft Lip Nasal Deformity. While primary cleft rhinoplasty has its advocates, correction of the septal deformity is generally performed during secondary rhinoplasty. The author describes the rationale for and the technique of Primary Caudal Septal Repositioning performed as an adjunct to cleft lip repair.

Learning Objectives:

After viewing this presentation, the learner will:

- Be familiar with the septal deformity associated with Cleft Lip Nasal Deformity.

- Have reviewed the rational for and technique of primary septal repositioning during primary cleft lip repair.

\section{TP04}

\section{ABDOMINAL WALL CLOSURE IN PEDICLE TRAM FLAPS}

\section{$M$ Haugrud}

A technique of abdominal wall closure using mesh placed within the rectus sheath. This facilitates muscle closure even if the fascia is of poor quality and covers the mesh in the event of skin flap complications.

\section{TP05}

VENOUS FLOW-THROUGH FLAP DESIGN AND HARVESTING TECHNIQUE

\section{A Islur}

Venous flow-through flaps represent a versatile soft tissue flap which can be harvested in 10-15 minutes and can be placed on the volar or dorsal surface of digits. Venous flow through flap indications, design and harvesting technique will be discussed.

\section{TP06}

\section{THE WAY I TREAT IMPOSSIBLE HAND INJURIES}

J Koay

PURPOSE: To share both the "Tips and Pearls" with our colleagues.

METHODS: Open wound treatments were carried out in these worst of the worst hand injuries.

RESULTS: Body parts were saved without further surgical complications.

DISCUSSION AND CONCLUSIONS: a) Open wound treatment

for grossly contaminated wounds.

b) Intravenous antibiotics should be given.

c) Save the skin for wound coverage.

Learning Objectives:

- The participants will be able to make quick decisions regarding treatment.

- The participants will be able to prevent further loss of tissue and infection.

\section{TP07}

\section{THE DINGMAN GAG AND PALATE SURGERY: "STOP KINKING MY TUBE!" \\ L Ross}

BACKGROUND: The dingman gag is used exclusively during cleft palate surgery at our centre. An approximate 20\% incidence of kinked tubes was witnessed.

PURPOSE: To share a modification which has completely eliminated this side effect with 5 years of use.

\section{TP08}

\section{IMMEDIATE BREAST RECONSTRUCTION USING IMPLANTS FOLLOWING THERAPEUTIC OR PROPHYLACTIC MASTECTOMY IN PATIENTS WITH PTOTIC BREASTS \\ CG Scilley}

Reconstructing a ptotic breast in a single stage with an implant alone is difficult. Using a "wise" type pattern for the mastectomy incision allows for reduction/reshaping of the skin envelop. Sewing the de-epithelialized inferior skin to the elevated lower border of pectoralis major creates a large pocket. The incision is closed as an inverted "T".

Learning Objectives:

- Use of a modified mastectomy incision to facilitate reconstruction of the ptotic breast using an implant.

- Use of a dermal muscular pocket for implant coverage combined with a breast reduction type incision to safely achieve a reasonable shaped breast following mastectomy.

\section{4}

\section{Canadian Expert Series}

\section{COMPLEX PIP INJURY - WHAT IS IN THE TOOLBOX}

\section{CW Watters}

The presenter will discuss PIP injuries using a Problem, Principle, Procedure format. The injuries include the author's approach to Boutonniere deformity, unstable intra-articular fractures, complex periarticular fractures and Poker Finger using static and dynamic splints, extension block k-wire, micro ORIF and implant arthroplasty, as indicated.

Learning Objectives:

After this presentation, the learner should be able to: 
- Inventory the injured PIP components and prioritize treatment options.

- Critically assess the utility of the variety of special splints and techniques presented.

- Consider further development needed in the treatment of complex PIP injuries.

\section{5}

THE TREATMENT OF PERIUNGUAL SQUAMOUS CELL CARCINOMA WITH MOHS MICROGRAPHIC SURGERY DA Graham, D Nickerson, E Campbell, JP Arlette

PURPOSE: To evaluate the treatment of squamous cell carcinoma (SCC) of the periungual region by Mohs Micrographic Surgery (MMS).

METHODS: Between 1997 and 2009 twenty two patients were seen for management of SCC of the periungual region. All lesions were treated by MMS, a method of histologically controlled removal of cutaneous malignancy. Records were evaluated for patient demographics, lesion location, histological pattern, and surgical data. Records were searched for lesion type, bony exposure, recurrence, previous treatment, and lesion duration prior to diagnosis. Long-term follow-up was by patient examination or survey.

RESULTS: The initial cure rate was $86 \%$. Recurrences occurred in 3 patients, who underwent a second MMS with longstanding cures of greater than 5 years. In 14 patients, defects were allowed to heal by secondary intention; 8 were reconstructed by amputation at the proximal or distal phalynx with preservation of volar tissues. Each type of repair was well tolerated, preserving good function and sensation of the finger.

CONCLUSIONS: There are both surgical and non-surgical therapies for periungual SCC. Non-surgical treatments (cryotherapy, Imiquimod, laser therapy) may only be suitable for superficial lesions, and can carry recurrence rates upwards of $50 \%$. Surgical options, such as wide local excision and amputation of the distal finger, sacrifice sensate volar tissues, and limited surgical excision has a recurrence rate of 56\%. MMS allows for both a maximal cure rate and preservation of sensate tissues, whilst giving excellent tumour control and removal. Our case series shows that this is a treatment with low morbidity and recurrence, while maintaining good form and function of the affected distal finger.

Learning Objectives:

- Participants will become more familiar with periungual SCC and the treatment options available.

- Participants will appreciate that MMS is an effective treatment for patients with this lesion.

\section{6}

\section{COMPARISON OF TRAPEZIECTOMY AND TRAPEZIECTOMY WITH LIGAMENT RECONSTRUCTION AND TENDON INTERPOSITION (LRTI): A SYSTEMATIC LITERATURE REVIEW}

\section{YK Li, C White, A Thoma}

PURPOSE: Currently, trapeziectomy with LRTI is the most commonly used technique for operative treatment of trapeziometacarpal osteoarthritis. However, it is uncertain whether the addition of LRTI to simple trapeziectomy confers any advantage. The purpose of this study was to systematically review the literature and determine which procedure, trapeziectomy or trapeziectomy with LRTI, offers the best results.

METHODS: A literature search was undertaken of the following electronic databases: Cochrane, AMED, EMBASE, HAPI, Healthstar, MEDLINE, TRIP, and Proceedings First (2002-Dec 2009). Studies were selected by two independent assessors if: (1) the study population included patients with basal thumb joint osteoarthritis and (2) the study was a comparative study or systematic review comparing trapeziectomy and trapeziectomy with LRTI. Inter-rater variability was assessed. Included studies were assessed using the Assessment of Multiple Systematic Reviews tool and Jadad scale, and objective (ROM, grip strength, pinch strength, health cost, postoperative complications) and subjective (pain relief, hand function, overall satisfaction, and quality of life) outcomes were extracted. Statistical analyses were performed with available data.

RESULTS: Two systematic reviews and five RCTs were identified and included in our review. Kappa for inter-rater agreement was 1.0. There were no significant differences between the two procedures at 12 months postoperatively in terms of palmar abduction $(\mathrm{p}=0.81)$, radial abduction $(p=0.22)$, grip strength $(p=0.77)$, tip pinch strength $(p=0.48)$, key pinch strength $(p=0.75)$, resting pain $(p=0.26)$, subjective weakness $(p=0.40)$, and subjective stiffness $(\mathrm{p}=0.92)$. In addition, neither procedure demonstrated superiority in terms of postoperative pain VAS score $(p=0.34)$, DASH score $(\mathrm{p}=0.75)$, and number of complications $(\mathrm{p}=0.13)$. No studies reported health costs.

CONCLUSIONS: Neither procedure produced greater benefit in terms of objective and subjective outcomes.

Learning Objectives:

- Participants will be able to identify current treatments for trapeziometacarpal osteoarthritis.

- Participants will learn the current evidence for the surgical treatment of trapeziometacarpal osteoarthritis.

\section{Residents Corner}

17

\section{A SYSTEMATIC REVIEW OF PROXIMAL INTERPHALANGEAL JOINT ARTHROPLASTY}

\section{K Chan, B Ayeni, L McKnight, A Thoma}

PURPOSE: To systematically review the literature and determine which arthroplasty option, if any, offers the best outcomes for patients with arthritis of the proximal interphalangeal (PIP) joint of the hand.

METHODS: A computerized search was conducted to identify studies evaluating arthroplasty techniques at the PIP joint for the treatment of arthritis. The data extracted from each trial included: active arc of motion, pain relief, and complication rates.

RESULTS: Two hundred and ten citations were identified, of which 42 were relevant articles and were included in the final analysis. High quality study design studies were not found in this review. Ten different arthroplasties were identified with the silicone implant being the most commonly studied. All arthroplasties demonstrated an improvement in postoperative joint motion, with the exception of the Flatt prosthesis. A more functional range of motion was achieved postoperatively in all arthroplasties, with the exception of the silicone-Dacron implants. Although most studies found that arthroplasty offers satisfactory pain relief, there was great variability in outcome measurement, making comparisons difficult. Similarly, the reporting of complications varied considerably. Overall, the silicone implants had the lowest rate of revision and salvage procedures.

CONCLUSIONS: Based on the available low grade evidence, silicone implant arthroplasty appears to be the most appropriate option. Confirmatory evidence is required from future studies that utilize more rigorous study designs, validated quality of life scales, and economic evaluations.

Learning Objectives:

At the end of this presentation, the learner will be able to:

- Familiarize themselves with the various arthroplasty techniques for the PIP joint.

- Understand their indications and limitations.

\section{8}

\section{PALMAR FASCIA CLOSURE - A MODIFICATION OF THE LIMITED OPEN CARPAL TUNNEL RELEASE TECHNIQUE}

\section{FB Loiselle, A Lin}

PURPOSE: Carpal tunnel release (CTR) is one of the most commonly performed elective hand surgeries in the developed world. Several techniques have been described from minimally invasive endoscopic to completely open techniques. Most techniques share similar success rates, where they differ is in post-operative recovery and risk of complications. We propose a modification of the limited open technique that 
mimics the advantages of the endoscopic technique without the increased risk of recurrent motor nerve injury.

METHOD: Patients were randomized to receive CTR with or without closure of the palmar fascia. The surgeon (AL) and independent evaluator were blinded. Patients who met electrophysiologic and clinical criteria for CTR surgery and who provided consent were enrolled in the study. Patients treated as part of a workman's compensation board claim or less than 18 years of age were excluded. A pre-operative assessment was performed within two weeks of surgery which included a Levine symptom severity score, functional status assessment, carpal tunnel provocative testing, grip and pinch strength measurement and pillar pain grading. Patients were reassessed at 2, 6, 12, and 24 weeks.

RESULTS: 51 CTRs were performed on 41 patients. Palmar fascia closure significantly reduced pillar pain up to 12 weeks post-operation $(p=0.04)$ but made no difference at 24 week follow up $(p=0.28)$. A trend toward improved functional status up to 12 weeks post-operation was also observed with closure of the palmar fascia $(p=0.10)$.

CONCLUSIONS: Closure of the palmar fascia during a limited open CTR reduces pillar pain and likely improves patient functional status. This technique also allows direct visualization of the recurrent motor branch.

Learning Objectives:

- The participant will be able to identify the proposed theories of pillar pain.

- The participant will be able to define the role of palmar fascia closure in their practice.

\section{9}

\section{THE USE OF IV HEPARIN IN DIGITAL REPLANTATION AND REVASCULARIZATION: THE QUEBEC PROVINCIAL REPLANTATION PROGRAM EXPERIENCE}

\section{Y Tahiri, V St-Supery, J Sampalis, PG Harris, G Landes, L Lessard,} A Nikolis

BACKGROUND: Anticoagulation administration in the early postoperative period can improve digital replantation outcomes. No consensus exists among microsurgeons regarding type of thromboprophylaxis regimens to be used. The current experience of the Provincial Replantation Center in Quebec was reviewed over a 4-year period.

METHODS: An initial retrospective review of all revascularized or reimplanted digits at our Replantation Center from April 2004 to April 2006 was conducted. Then, data of all patients, with devascularized/ amputated digits, treated at our center from January 08 to January 09 were prospectively collected. The two cohorts were compared with regards to demographics, injury characteristics, medication used for post-operative thromboprophylaxis, as well as complication and success rates. Proportions were compared using $\chi^{2}$ tests / Fischer's exact tests. Multivariate analysis was conducted with logistic regression.

RESULTS: 175 digits were treated between April 2004 and April 2006: 104 revascularizations and 71 amputations. Intravenous (IV) heparin was used in $35.1 \%$ of the cases and was associated with a 3.59 -fold (95\% CI, 1.55-8.31) increase probability of developing a complication $(\mathrm{p}=0.001)$. In 2008, 106 digits were treated: 45 revascularizations and 61 total amputations. IV heparin was used in $14.6 \%$ and was not significantly associated with a higher complication rate $(p=0.612)$. The success rate between the two cohorts was similar $(p=0.557)$. The number of complications decreased from the first period $(20.6 \%)$ to the second one $(12.7 \%)$.

CONCLUSION: IV heparin use following digital replantation and revascularization is not warranted. Surgical technique and type of injury remains the most important predictors for success in these complex procedures.

Learning Objectives:

- Clarify the role of anticoagulation following digital replantation/revascularization with respect to complication and success rates. Give recommendations regarding post-operative anticoagulation therapy following these complex procedures.

\section{0}

\section{THREE-DIMENSIONAL ANATOMICAL STUDY OF THE VASCULAR SUPPLY TO THE TENSOR FASCIA LATA AND OVERLYING SKIN}

K Almutairi, M Tang, SF Morris

BACKGROUND: The tensor fascia lata muscle and its overlying skin region are frequently harvested as musculocutaneous, fasciocutaneous and/or perforator skin flaps due to a consistent and large vascular supply from lateral circumflex femoral artery (LCFA). Recently, we have used 3-D vascular injection studies to clarify the vascular anatomy of flaps throughout the body. The objective of the current study is to describe the territorial blood supply of the LCFA through the TFL muscle and its overlying skin paddle and to document the various options for creative flap design in the area.

METHODS: Seven cadavers were injected with a modified lead oxidegelatin mixture and underwent three-dimensional reconstruction using a spiral computed tomography scanner and the MIMICS (a software program). Dissection, angiography, and photography of each layer of dissection were performed to outline the course of the lateral circumflex femoral artery. Exact volumetric measurements of the intravascular blood supply of the TFL and the diameter of the pedicle and its terminal branches to skin were recorded.

RESULTS: A total of 14 TFL muscles were dissected and analyzed. In 12 out of the 14 samples (85.7\%), the lateral circumflex femoral artery (LCFA) was a direct branch of the profunda femoris artery. In the remaining specimens, the LCFA branched directly from the femoral artery. All 14 muscles had constant $3+/-2$ musculocutaneous perforators. In 1 out of the 14 muscles there were no septocutaneous branches $(7.8 \%)$. The average volume of muscle supplied by the ascending branch of the ascending lateral circumflex femoral artery to the TFL muscle in volume was $50+/-2.6 \mathrm{~cm}^{3}$. The average surface area of muscle supplied by the ascending branch of the LCFA was $111.7+/-2 \mathrm{~cm}^{3}$.

CONCLUSIONS: This is the first report of an anatomical technique documenting the intramuscular blood supply to a skeletal muscle by a novel three-dimensional technique, this technology allows precise measurement of the vasculature and provide detailed information regarding anatomical landmarks, diameters and lengths of intramuscular and extramuscular blood supply.

Learning Objectives:

- Learn about a new modality to describe the anatomy of TFL flap in detail.

- Describe the variation of the skin perforators overlying the TFL muscle.

- Provide quantifiable data regarding the variation in flap anatomy.

\section{1}

\section{VALIDITY OF THE DASH FOR FUNCTIONAL OUTCOMES EVALUATION IN UPPER EXTREMITY SARCOMA LIMB SALVAGE THERAPY}

J Robinson, C Temple, N Schachar, W Temple

PURPOSE: To validate the Disability of the Arm, Shoulder, and Hand (DASH) questionnaire in patients undergoing limb salvage surgery for upper extremity sarcoma.

METHODS: Twenty-eight patients underwent neoadjuvant chemoradiation therapy, surgical resection and flap reconstruction for upper extremity sarcoma. Each patient completed the Toronto Extremity Salvage Score (TESS) and DASH questionnaires postoperatively. Functional outcome using the Muskuloskeletal Society Tumour Score (MSTS) was also rated by two independent clinical assessors. Demographic data, tumour characteristics, and treatment information were collected. Criterion validity of the DASH was evaluated by Pearson Correlation Coefficient with the TESS and mean MSTS scores. Construct validity of the DASH and TESS was determined by univariate analysis with predictive factors for sarcoma limb salvage outcome. RESULTS: Regarding criterion validity, the DASH correlated highly with the TESS $(-0.873, \mathrm{p}<0.001)$, and the mean MSTS $(-0.793, \mathrm{p}<0.001)$. The MSTS intraclass correlation coefficient was high, at 0.774 [0.563 - 0.890]. Regarding construct validity, neither the DASH nor 
TESS demonstrated statistically significant results with known sarcoma predictive factors: tumour recurrence (DASH $p=0.95$; TESS $p=0.49$ ), more distal tumours (DASH $\mathrm{p}=0.35$; TESS $\mathrm{p}=0.19$ ), chemoradiation complications (DASH $\mathrm{p}=0.38$; TESS $\mathrm{p}=0.57$ ), tumours larger than $5 \mathrm{~cm}(\mathrm{DASH} \mathrm{p}=0.52$; TESS $\mathrm{p}=0.57)$, and lower grade tumours (DASH $\mathrm{p}=0.71 ;$ TESS $\mathrm{p}=0.91$.

CONCLUSIONS: The DASH demonstrated high criterion validity for use in functional outcome assessment of patients with upper extremity sarcoma undergoing limb salvage surgery, when using the TESS and MSTS as the gold standard. Neither the DASH nor the TESS demonstrated high construct validity in our patient population.

Learning Objectives:

- To understand the usefulness of the DASH in evaluating outcome for patients with upper extremity sarcoma surgery.

\section{2}

\section{A.W. Farmer Lecture}

\section{THE DEVELOPING SCIENCE BEHIND STRUCTURAL FAT GRAFTING TECHNIQUE \\ Sydney R Coleman, MD}

A brief review of the technical aspects for the basis of harvesting, purification and placement will be followed by a look at the scientific and anecdotal evidence for using this specific technique. In particular, effect of centrifugation on harvested fat will be discussed; and the first studies of the growth factors and primitive cells residing in different densities of grafted fat will be presented.

Our recent increased understanding of stem cell activity in normal adipose tissue leads one to believe that such dramatic aesthetic improvements of skin overlying transplanted fat may at least in part be an effect of underlying stem cell activity.

Discussion will be made of the potential effects of adipose derived stem cells on the repair of the surrounding tissues

The mechanism of fat graft improvement in surrounding tissue is not clear, and the role of adipose derived stem cells and preadipocytes in fat survival and repair remains to be determined. Research has indicated the possible involvement of more undifferentiated (primitive) cells in some of the observed effects of fat grafting on surrounding tissues. Of particular interest is the research that has pointed to the use of stem cells to repair the tissues around which fat is placed especially after therapeutic radiation.

Learning Objectives:

- To examine scientific studies concerning the technique of structural fat grafting.

- To relate methods of improving predictability of fat grafting based on these studies.

- To relate recent studies on the effect of grafting fat on surrounding tissue focusing on the effect on therapeutic radiation injury.

Disclosure of Interest:

Dr. Coleman has noted the following:

- I have the potential for stock options with Cytori Therapeutics and Brava.

- I do research into techniques using products from Mentor.

- I receive royalties and consulting fees from Mentor.

\section{Eye-Opener Session}

\section{SURGICAL TREATMENT OF MIGRAINE HEADACHES}

\section{Bahman Guyuron, MD}

Migraine headache is a chronic, often incapacitating condition that afflicts millions of people, disproportionately affecting more females than males. Despite its prevalence, this condition remains largely under diagnosed and under treated. This presentation will educate participants in the surgical techniques that have been developed over the last 10 years to treat the four most common migraine headache trigger sites with a success rate of approximately $90 \%$. An in-depth overview of the symptoms and diagnosis of migraines, identification of trigger sites with Botox, and relevant anatomy will be presented.
At the end of this course you will be able to:

- Review symptoms $\mathfrak{E}$ diagnosis of migraine headaches.

- Discuss the constellation of symptoms.

- Discuss potential pathophysiology of trigger sites.

- Injection of Botox as treatment.

- Preop work up and selection of surgical technique.

- Detailed surgical treatment of migraine headaches.

- Management of adverse effects.

- Pertinence of this information to the plastic surgeon.

Disclosure of Interest:

Dr. Guyuron has noted the following:

- He is a shareholder in Noteworthy Electronic Medical Record and owner of Innovative Medical Equipment.

- He is editor of Plastic Surgery Indications and Practice.

\section{Residents Corner}

\section{3}

\section{EFFECTS OF CHRONIC IMPLANTATION OF A NOVEL NERVE CUFF ON PERIPHERAL NERVE}

\section{Korus, L Gan, K Wong, K Todd, J Olson, M Morhart,}

A Prochazka

PURPOSE: Functional electrical stimulation of peripheral nerves can be used in spinal cord injury to regain limb movement. The "Stimulus Router System" is a design that takes advantage of implanted leads which capture current flowing between external electrodes and routes this current to a target nerve via a cuff. Such cuffs, however, have potential to cause neural damage over time. We set out to determine the effects of chronic implantation with a novel cuff design currently being used in human experiments.

METHODS: Seven rabbits were implanted, each with two nerve cuffs (on common peroneal and posterior tibial nerves). Both cuffs were placed on the same side of the animal and the contralateral side was subjected to a sham operation. Leads attached to the cuff were tunneled to subcutaneous tissue on the back of the rabbit. External electrical stimulation was applied and muscle activation thresholds were recorded at $0,1,3$ and 7 months. Following this animals were sacrificed and common peroneal and posterior tibial nerves (both cuff and sham sides) were analyzed histologically.

RESULTS: Stimulation thresholds were highest at 1 month postimplantation. This difference was statistically significant in the common peroneal nerve group $(\mathrm{p}=0.0224)$. Subsequent stimulations at 3 and 7 months saw decreasing thresholds, however these thresholds never returned to baseline, as seen in the immediate post operative period. These results are similar to human data as seen by Olson et al. Histological analysis of the nerves is pending.

CONCLUSIONS: Tissue reactions to nerve cuffs and pick up leads may result in increased stimulation thresholds, however these have a tendency to decrease over time.

Learning Objectives:

- The learner will become familiar with use of functional electrical stimulation and the potential effects implantable leads can have on peripheral nerves.

\section{4}

\section{AXON COUNTS OF POTENTIAL NERVE TRANSFER DONORS FOR PERONEAL NERVE RECONSTRUCTION} CP White, MJ Cooper, CM Levis

PURPOSE: The purpose of this anatomic study is to identify potential donors of motor axons from the tibial nerve.

METHODS: Using human cadaveric lower extremities, all motor nerve branches of the tibial nerve were identified, isolated and biopsied. As a comparison of axon counts, sections of the motor branches of the Deep Peroneal Nerve (DPN) were harvested and prepared. Biopsy specimens were then processed and stained using glutaraldehyde, epoxy resin and toluene blue. The axons from each nerve specimen were quantified using a Metamorph scanner and manual counting of all axons in a maximum of 
three sections for each motor branch. Mean and standard deviation of axon counts for each nerve branch were calculated.

RESULTS: The motor branch to the tibialis anterior muscle had on average a cross sectional area of $0.270 \mathrm{~mm}$ and an average of 3999.7 axons. This was most similar in cross sectional area to the branch to flexor hallucis longus $(0.262 \mathrm{~mm})$ and in total number of axons to the branch to popliteus (3036.3).

The motor branch to extensor hallucis longus was found to have an average cross sectional area of $0.250 \mathrm{~mm}$ and an average of 2236.9 axons. This was most similar in cross sectional area to flexor hallucis longus $(0.262 \mathrm{~mm})$ and total number of axons to the branch to medial gastrocnemius (2679).

CONCLUSIONS: This study showed several variations in nerve anatomy with a variety of branch patterns and numbers of nerve bundles. Ideal donor nerves were identified. As well, secondary potential donors also appear to be available with similar axon counts. All potential donors should be considered when selecting branches for transfer without sacrificing function for the entire muscle group. Limited sample size and confounding issues with sample size as well as co-morbidities of patients and counting errors are sources of error in the study.

We believe that nerve transfers should be included in the armamentarium for lower extremity re-innervation as it is in the upper limb. The ultimate goal is to select appropriate and expendable motor nerves to reinnervate the ankle $+/$ - the toe extensors that may lead to more predictable and functional results in reconstructing the DPN than that achieved by nerve grafts.

Learning Objectives:

- Participants will be able to define common peroneal nerve injuries.

- Participants will be able to describe treatments for common peroneal nerve injuries.

- Participants will be able to identify the most similar transferable nerves to the deep peroneal nerve.

\section{5}

\section{CHARACTERIZATION AND QUANTITATION OF DIFFERENTIATION AND CAPACITY OF MESENCHYMAL STEM CELLS TO SUPPORT PERIPHERAL NERVE REGENERATION}

\section{A Ladak, J Olson, T Gordon, E Tredget}

PURPOSE: Functional recovery after peripheral nerve injuries is limited by time and distance required for axon regeneration. Deterioration of denervated Schwann cells (SCs) is the key limiting factor in this recovery. Our objective was to explore the potential for mesenchymal stem cells (MSCs) to replace SCs for support of axon regeneration. Our aims were to characterize and quantify MSC differentiation into a SC-like phenotype and to evaluate their functional capacity to promote neurite and axon outgrowth in vitro and in vivo.

METHODS: MSCs isolated from bone marrow of Sprague-Dawley rats were characterized by plastic adherence and pluripotency towards mesodermal lineages. Isolated stem cells were then stimulated towards a SC phenotype using specific growth factors, and cell marker analysis was performed to verify SC phenotype. The neurotrophic effect of these differentiated stem cells was assessed in vitro using an indirect co-culture system with rat dorsal root ganglion cells (DRG). Finally, the effect of dMSCs in vivo was assessed using a rat sciatic nerve model.

RESULTS: Differentiation resulted in positive immunocytochemical staining for the SC markers glial fibrillary acidic protein (GFAP), S100, and nerve growth factor receptor (NGFR). Using an indirect co-culture system, we compared the ability of dorsal root ganglion (DRG) cells to extend neurites in indirect contact with undifferentiated MSCs (uMSCs) and differentiated MSCs (dMSCs) as compared to SCs. The mean values of the longest length of the DRG neurites was the same for the dMSCs and SCs and significantly higher than the uMSC and DRG alone culture systems $(\mathrm{p}<0.05)$. In vivo, dMSC transplantation resulted in a greater number of motoneurons $(627.75 \pm 86.02)$ extending axons across a nerve gap than an empty conduit (284.83 \pm 48.11$)$.

CONCLUSIONS: We conclude that bone marrow-derived MSCs differentiate into a SC-phenotype that expresses SC markers transiently, sufficient to support neurite outgrowth in vitro and axonal regeneration equivalent to that of SCs in vitro and in vivo.

Learning Objectives:

- Following this presentation, the participant will have learned (1) inherent problems in peripheral nerve regeneration and (2) the possible application of stem cell therapy to enhance regeneration across nerve gaps.

\section{6 \\ TENSION AND TISSEEL: HOW DO IN VITRO PERIPHERAL NERVES HOLD UP?}

\section{A Card, J Bain}

PURPOSE: Peripheral nerve repairs have the best outcomes with tensionless repairs. Previous In Vivo experiments have shown that increased tension results in irregular scar formation. Our purpose was to assess if fibrin sealant had any effect on nerve healing at different tensions.

METHODS: 180 sciatic nerve segments were randomized into 5 groups of varying tension. Three subgroups were created; controls (C), microsurgical repairs $(\mathrm{R})$, and repairs with Tisseel (T). All microsurgical repairs were completed in standard fashion (9-0 nylon epineural repair under the operating microscope). All nerves were incubated in neural tissue culture media for 14 days. Nerves were fixed and stained to identify specific tissue components. Slides were digitized and tissue composition analyzed by computerized algorithm (Sigma Scan Pro). Groups were compared with ANOVA and post hoc Tukey tests $(\mathrm{p}<0.05)$.

RESULTS: Repair failure rates were more common in the $\mathrm{R}$ group $(p=0.06)$ and days-to-failure was significantly longer in group $T$ $(p=0.009)$. Nerve elongation was decreased in the T group $(p<0.001)$. A qualitative histological analysis was performed to assess the difference in healing patterns. Both the total amount and distribution of nervous tissue were decreased in the R group $(p<0.001, p<0.0001)$. The amount of fibrous tissue increased with tension in all groups $(\mathrm{p}<0.05)$. Axonal tissue decreased in the $\mathrm{C}$ group compared to the $\mathrm{T}$ group where it remained relatively constant $(\mathrm{p}<0.001)$.

CONCLUSIONS: Peripheral nerve healing In Vitro resulted in increased internal fibrotic tissue with increased tension. The addition of fibrin sealant to repaired nerve segments decreased the degree of elongation and increased the number of days before repair failure. Furthermore, fibrin sealant mitigated the loss of both axonal and supporting nervous tissue.

Learning Objectives:

- To understand the effect of tension on nerve healing.

- To explore the effects of fibrin sealant on axonal and connective tissue in repaired nerves under tension.

\section{7}

\section{CORRELATING SPYR IMAGING (ICG FLUORESCENCE ANGIOGRAPHY) WITH FLAP OUTCOMES}

DS McDonald, JR Friesen, MG Sowa, EW Buchel, L Sigurson, TEJ Hayakawa

PURPOSE: The Novadaq SPYR imaging system utilizes indocyanine green (ICG) to allow real time images of the microcirculation following administration of an intravenous bolus. The purpose is to evaluate SPYR imaging in predicting necrosis in a reverse McFarlane skin flap model before clinical signs are present.

METHODS: Reverse McFarlane skin flaps were raised in 9 rats. ICG perfusion imaging was performed immediately following and 1 hour after surgery. Digital pictures were taken immediately, at 1 hour, and 72 hours. Customized software allowed one investigator, blinded to the ICG results, to outline the anatomical border of the flap and eventually the limits of demarcation on the $72 \mathrm{~h}$ picture. A binary image was saved where the flap was segmented into two parts (survived and died). Immediate and 1 hour accumulated fluorescence intensity (AFI) images were then evaluated against the binary image. AFI, normalization and image registration were performed using MATLAB (version 7.5).

The non-parametric estimator of Area Under the Curve (AUC) was used to test the ability of accumulated fluorescence intensity to predict tissue viability. AFI was determined for each pixel in the ICG image 
which in turn corresponded to a pixel in the binary image of the flap. Regions that were viable $72 \mathrm{~h}$ after surgery were assigned a value of 1 , dead regions a value of zero. Percentile bootstrap 95\% confidence intervals (CI) were estimated by rank ordering 2000 estimates of AUC with each estimate based on 5000 random selected pixels.

RESULTS/CONCLUSION: SPYR imaging was an excellent predictor of flap viability when measured $1 \mathrm{~h}$ after raising the flap with a $95 \% \mathrm{CI}$ of AUC [0.949, 0.961]. Immediate imaging was a fairly good predictor [0.886, 0.904].

Learning Objectives:

- Understand the utility of SPYR imaging in predicting flap necrosis in the rat model.

\section{8}

\section{ROLE OF WNTS AND CHARACTERIZATION OF WNT SIGNALING PATHWAYS DURING DE NOVO BONE FORMATION IN A WILD-TYPE MOUSE MODEL OF DISTRACTION OSTEOGENESIS}

\section{LA Alsalmi, ZS Haidar, A Izadpanah, M Tabrizian, RC Hamdy,}

LM Lessard

PURPOSE: Wnts are known to have a role not only in bone mass control but in a different array of processes. However, the involved pathway(s) is not yet fully understood and was never studied in cases of distraction osteogenesis (DO). Characterizing Wnt signaling pathway during DO is essential and might eventually enhance the clinical DO outcome specifically via acceleration of bone consolidation post-lengthening using novel approaches to protein therapy.

METHOD: Wnt signaling expression was investigated in 60 adult female wild-type mice that have received right tibial DO. Following the osteotomy and mounting of custom-made miniature Illizarov rings, distraction began after a latency period of 5 days at a rate of $0.2 \mathrm{~mm} / 12 \mathrm{~h} / 2$ weeks. Animals were divided into 5 groups according to the time of sacrifice: day 5 (end of latency); days $11 \& 17$ (mid \& end of distraction); and days $34 \& 51$ (mid \& end of consolidation) for interphase examination. The harvested bony regenerate then underwent complete microarray, histological and immunohistochemical analysis.

RESULTS: Overall, data show a statistically significant Wnt signaling up-regulation during distraction compared to the latency and consolidation phases. More specifically, over-expression of Wnts 4, 5b and 16 were evident at the end of the distraction phase. Wnt receptors Fzd 1, 2 and 9 along with Wnt inhibitors NKd2, Dkk3 and $\beta$-catenin and transcription factors ctbp1 and ctbp2 were also up-regulated on day 17 post-osteotomy. Interestingly, the Wnt inhibitor Dkk1 was under-regulated.

CONCLUSIONS: Manipulating the Wnt signaling pathway during the early phases of DO seems most appropriate for enhancing de novo bone formation. Our ongoing studies focus on the stimulation of Wnt signaling alongside localized and release-controlled bone growth factor therapy in long bone - as well as mandibular DO.

Learning Objectives:

- Understand the clinical potential and current limitations of distraction osteogenesis.

- Understand Wnts and the involved Wnt receptors, inhibitors and transcription factors during new bone regeneration in fracture healing and criticalsized defects.

- Highlight novel alternative approaches to the acceleration of bone regeneration.

\section{9}

\section{HYPERTROPHIC SCAR FORMATION IN THE NUDE MOUSE ANIMAL MODEL}

\section{Momtazi, C Anderson, J Ding, E Tredget}

PURPOSE: Hypertrophic scar (HSc) and keloids represent the dermal equivalent of fibroproliferative disorders. Lack of a relevant, practical animal model is a barrier towards a better understanding of the mechanisms involved in these processes. The objective of this study is to determine whether transplanting human skin onto nude mice results in wounds similar grossly and histologically to human HSc.
METHODS: $2 \times 1.5 \mathrm{~cm}$ biopsies of human split thickness abdominal skin were grafted onto the backs of twenty nude mice. Wounds were digitally photographed weekly to document healing. Animals were sacrificed at 30, 60,120 and 180 days. At these time points, scar biopsies as well as normal skin samples were harvested. Histological analysis consists of scar thickness using a micrometer, Real Time Polymerase Chain Reaction (RT-PCR) to quantify Type I collagen messenger Ribonucleic Acid (mRNA), immunohistochemistry for Transforming Growth Factorbeta (TGF- $\beta$ ) and Connective Tissue Growth Factor (CTGF) and staining for fibrocytes. Engraftment of human skin was confirmed by staining keratinocytes with anti-human, Human Leukocyte Antigen (HLA)ABC Ab.

RESULTS: Preliminary results reveal that nineteen of the twenty mice (95\%) demonstrate firm, elevated wounds similar to human HSc. Histologically, these wounds are thicker and possess an abundance of disorganized, whorl-like collagen fibers. Average scar thickness, up to day 60 , is $508 \pm 21 \mu \mathrm{m}$, compared to $149 \pm 4 \mu \mathrm{m}$ for normal skin controls (paired t-test, $\mathrm{p}=0.0001$ ).

CONCLUSIONS: Grafting split thickness human skin onto nude mice results in wounds that are similar macroscopically and histologically to human HSc. This model represents a potential tool for observing and investigating HSc formation.

Learning Objectives:

Participants will be able to:

- Support the need for a relevant animal model for HSc.

- Identify and describe histopathological features distinguishing normal skin from HSc.

- Consider the nude mouse as an animal model for investigating HSc.

\section{0}

\section{OVEREXPRESSION OF DC109 IN THE EPIDERMIS REDUCES SCARRING IN A BLEOMYCIN INDUCED MODEL OF SKIN FIBROSIS}

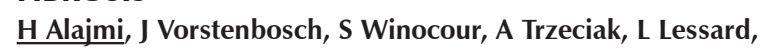
A Philip

PURPOSE: To determine the effects of overexpressing CD109 in mouse epidermis on TGF-Beta signalling and ECM production in vivo. METHODS: Skin fibrosis was induced in mice overexpressing CD109 in the epidermis and wild-type littermates by giving intradermal injections of bleomycin or PBS control on alternating days for 28 days. Mice were subdivided into groups to be sacrificed 14, 21 and 28 days after the initial injection and tissues were harvested for analysis. We assessed histological and biochemical changes by analyzing Masson's Trichrome and $\mathrm{H} \& \mathrm{E}$ stained skin tissue and changes in ECM and TGF-beta signalling proteins by western blot.

RESULTS: Intradermal injections of bleomycin increase ECM deposition in the skin of both wild-type and transgenic mice overexpressing CD109. After 28 days of bleomycin injections, the transgenic mice display reduced dermal thickness compared to wild-type littermates $(p<.005)$. Additionally, the transgenic mice express reduced levels of fibronectin and phoshoSmad 2 compared to wild-type littermates upon bleomycin injection.

CONCLUSION: CD109, when overexpressed in the epidermis, promotes a reduction of dermal thickness and improved collagen organization in a bleomycin-induced model of fibrosis. Collectively, our data illustrate the potent regulatory effect of CD109 on skin fibrosis, and suggest that this molecule may have therapeutic value for the treatment of skin disorders such as hypertrophic scarring and scleroderma.

Learning Objectives:

- Bleomycin injection enhances collagen deposition in both wild-type and transgenic mice. Transgenic mice display improved collagen organization and reduced dermal thickness as compared to wild-type littermates. Transgenic mice exhibit decreased TGF-Beta signaling and fibronectin expression as compared to wild-type mice. 
31

\section{DETERMINING THE STRUCTURAL INTEGRITY OF DECELLULARIZED TRACHEAL ALLOGRAFTS}

\section{S Haykal, TK Waddell, S Hofer}

PURPOSE: Tracheal reconstruction is required following large circumferential defects that are not amenable to direct anastomosis. The indications for such reconstructions are malignancy, traumatic injury and tracheal stenosis. Airway allotransplantation has recently been described as a solution to this problem but involves risks related to the use of immunosuppression. Decellularization techniques have been used in many organs and allow for the use of functional matrices without the need for immunosuppression. The purpose of this study is to determine the structural integrity of decellularized tracheal allografts. This is the first objective of a two-fold study which aims at defining the immunogenicity of decellularized tracheal allografts.

METHODS: Tracheas were harvested from Yorkshire pigs. These matrices were decellularized using multiple cycles of two different detergent-enzymatic reactions described in the literature: Sodium deoxycholate + DNAse I (Protocol A) and Triton X-100 + Pronase + CHAPS (Protocol B). Native tracheas and decellularized tracheas were placed in a device, sealed at both ends and exposed to increasing static negative pressures. CT scan imaging of these tracheas at different negative pressures was used to plot the luminal area over the pressure to determine tracheal compliance.

RESULTS: Native tracheas $(n=6)$ and decellularized tracheas using Protocol A $(n=6)$ and Protocol B $(n=6)$ were exposed to increasing static negative pressures. Luminal area, volume and compliance were measured using CT Imaging. Native tracheas collapsed completely at a pressure of $-30 \mathrm{mmHg}$ with an average compliance of $1.58 \pm 0.08$. Following five cycles of decellularization, decellularized tracheas using Protocol A collapsed at a pressure of $-12 \mathrm{mmHg}$ with an average compliance of $0.8 \pm 0.2$ while tracheas using Protocol B collapsed at a pressure similar to that of native tracheas.

CONCLUSIONS: Decellularized tracheal matrices can be used for airway transplantation without the need for immunosuppression. Structural integrity appears to be maintained using protocols with neutral constituents (such as CHAPS). The correlation between maintenance of function and immunogenicity remains to be determined.

Learning Objectives:

- Explore different decellularization techniques and their biological properties.

- Determine the structural integrity of decellularized tracheal allografts.

- Understand the application of tracheal decellularization to the area of composite tissue allotransplantation.

\section{2}

\section{ROLE OF ACTIVATED PROTEIN C AND ITS RECEPTOR IN INHIBITION OF MELANOMA METASTASIS}

M Bezuhly, R Cullen, CT Esmon, SF Morris, KA West, B Johnston,

\section{RS Liwski}

PURPOSE: Metastasizing cancer cells are thought to overcome the endothelial barrier through mechanisms commonly observed in leukocyte trafficking, including the upregulation of adhesion molecules, apoptosis induction and disruption of normal endothelial intercellular contacts. Given the parallels between these processes, we hypothesized that a therapy directed at the endothelium to reduce leukocyte trafficking might also be of benefit in preventing tumour metastasis. Engagement of endothelial protein C receptor (EPCR) by activated protein C (aPC) decreases expression of endothelial adhesion molecules implicated in tumour-endothelium interactions. We examined the role of the aPC/EPCR pathway on tumour migration and metastasis.

METHODS: In vitro adhesion and transendothelial migration assays were performed using fluorescence-labeled B16-F10 melanoma cells plated on confluent endothelial monolayers pretreated with recombinant human aPC (rhaPC). In vivo, B16-F10 melanoma metastasis models were performed using transgenic EPCR overexpressing (Tie2-EPCR) and wildtype (WT) mice treated with or without rhaPC. Intravital imaging was performed to quantify cancer cell lodgement in the hepatic model.
Real-time polymerase chain reaction was performed to assess transcription levels of adhesion molecules, pro-inflammatory cytokines and apoptotic mediators.

RESULTS: In vitro, B16-F10 melanoma cells showed decreased adhesion to and transmigration through endothelium treated with recombinant human aPC (rhaPC). In murine B16-F10 metastasis models, transgenic EPCR overexpressing (Tie2-EPCR) mice exhibited improved survival and marked reductions in liver (50\%) and lung (92\%) metastases compared to wildtype (WT) animals. Intravital imaging revealed reduced B16-F10 entrapment within livers of Tie2-EPCR compared to WT mice. A similar reduction was observed in WT mice treated with rhaPC. Strikingly, rhaPC treatment resulted in a $44 \%$ reduction in lung metastases. This was associated with decreased lung P-selectin and TNF- $\alpha$ mRNA levels.

CONCLUSION: This study provides proof of concept for the strategy of using rhaPC in the prevention of tumour metastasis, and underscores the complex interplay between inflammation and cancer progression.

Learning Objectives:

- To appreciate the parallels between tumour metastasis and leukocyte trafficking.

- To understand the multiple cytoprotective and anti-inflammatory activities of activated protein $\mathrm{C}$ (aPC).

- To appreciate how the pleiotropic effects of aPC can be used to decrease tumour metastasis.

\section{3}

\section{HOW ACIDIC IS THE LIDOCAINE YOU ARE INJECTING?} SG Frank, DH Lalonde

PURPOSE: The infiltration of local anesthetics can be painful; some of this pain may be attributed to the high acidity of the anesthetic solutions. The $\mathrm{pH}$ of local anesthetics is often unknown by clinicians, and certainly underappreciated. Given the frequent use of local anesthetics by plastic surgeons, the unrecognized use of acidic, and hence painful, agents may present an opportunity for a change in practice and improvement in patient comfort by buffering the acidic solutions with inexpensive, readily available bicarbonate solution. This study determined the $\mathrm{pH}$ of commonly used anesthetic agents in our hospital.

METHOD: Commercially available solutions of $1 \%$ and $2 \%$ lidocaine, with and without epinephrine 1:100,000, were obtained from various settings throughout our tertiary care institution. Fifty samples of each solution, from a variety of manufacturers, lots and ranging in time to expiry, were measured for $\mathrm{pH}$.

RESULTS: Preliminary results were obtained from a limited sample of solutions. Mean $\mathrm{pH}$ for solutions of $1 \%$ plain lidocaine was $6.0 ; 1 \%$ lidocaine with epinephrine was $4.3 ; 2 \%$ plain lidocaine was $6.0 ; 2 \%$ lidocaine with epinephrine was 4.4. The lowest recorded $\mathrm{pH}$ was 2.98 in an expired sample of $1 \%$ lidocaine with epinephrine. Early trends indicate that increasing age of the solutions with epinephrine is associated with a significant decline in $\mathrm{pH}$.

CONCLUSIONS: Lidocaine without epinephrine is only somewhat more acidic than physiologic $\mathrm{pH}$. However, the buffering that is required for the stability of epinephrine in epinephrine-containing lidocaine reduces the $\mathrm{pH}$ to markedly acidic levels. As these solutions approach their expiry date they appear to become even more acidic.

Learning Objectives:

- Appreciate the acidity of some of the commonly used anesthetic solutions.

- Consider the high acidity of epinephrine-containing lidocaine and its possible impact on pain during infiltration.

- Be aware of the possible increased acidity of epinephrine-containing lidocaine with time.

\section{4}

THE RELATIONSHIPS BETWEEN CLEFT LENGTH, CLEFT WIDTH AND CLEFT TYPE IN CLEFT PALATE

\section{MH Mahoney, DM Fisher}

PURPOSE: It has been proposed that the greater the severity of the cleft of the palate, the worse the outcome. The primary objective of this 
study is to determine the relationship between palate length, cleft palate width and cleft type.

METHOD: A review of prospectively collected data on all consecutive primary palate repairs by a single surgeon, from January 2000 to January 2010, was completed. Patient demographics, Veau classification (I-IV), Kernahan \& Stark classification - primary/secondary palate $(\mathrm{CL} / \mathrm{P})$ \& secondary palate (C/P), Randall length (I-IV) and measurement of cleft width were analyzed for associations.

RESULTS: 485 patients underwent primary palatoplasties from January 2000 - January 2010. There were 276 males and 209 females; mean age was 20.4 months (range, 6.6 months - 17.7 years). Kernahan \& Stark Class included: $\mathrm{CP}=260$ and $\mathrm{CL} / \mathrm{P}=225$. Veau Class included: $\mathrm{I}=85, \mathrm{II}=$ 175 , III $=165, I V=60$. Randall length included: $I=81, I I=319, I I I=58$, $\mathrm{IV}=2$. Average cleft width was $7.7 \mathrm{~mm}$ (range, $0-19 \mathrm{~mm}$ ). Significant associations included: CP was associated with Randall Type I (longer palates), CL/P was associated with Randall Type III (shorter palates) $(p<0.0001)$. Randall Types II-IV had wider clefts than Randall Type I $(p<0.0001)$. Wider clefts were seen in the CL/P group $(p<0.0001)$. No difference in width was seen in unilateral and bilateral CL/P $(\mathrm{p}=0.0034)$. CONCLUSIONS: Patients with CL/P have wider palates than patients with CP, and are more often associated with Randall Types II \& III. Patients with CP are more often associated with Randall Types I \& II. Width and length is similar amongst unilateral and bilateral CL/P. Preoperative width and length may be used to explain the outcomes seen in varying degrees of cleft palate severity.

Learning Objectives:

- To describe the relationships between cleft palate length, width and type. This will provide the learner with further knowledge in the preoperative evaluation of and prognosis for patients with palatal clefts.

\section{5}

\section{A TEN-YEAR, TWO-INSTITUTION REVIEW OF PEDIATRIC DOG ATTACKS: ADVOCATING FOR A NATIONWIDE PROHIBITION OF DANGEROUS DOGS}

\section{FM Yau, JS Arneja, K Pappas, G Singh}

PURPOSE: Affectionately referred to as 'man's best friend', dog attacks in the pediatric population often test this analogy. Pediatric dog attacks are a significant public health issue and result in physical morbidity, psychological disability, and financial strains. We performed analysis of our cumulative two-institution pediatric dog attack data and offer evidence to support a nationwide prohibition of dangerous dogs.

METHODS: A retrospective review was performed at two urban Children's hospitals from 1996-2006 of all dog attacks presenting to the plastic surgery service. Charts were reviewed with analysis of patient demographics, injury site, operative intervention, and dog-specific data.

RESULTS: 109 patients were included for review. Mean age was 3.9 years (range $2-18$ years). $95 \%$ of patients required surgical intervention with $30 \%$ requiring a skin graft or flap reconstruction. $88 \%$ of dogs were known to the victim, $46 \%$ of attacks were provoked, and $57 \%$ of dogs were deemed to be of a dangerous breed (Pit Bull or Rottweiler). Mean hospital duration was 4.7 days and $27 \%$ required additional reconstructive plastic surgery. A representative case of a 4-year old dog attack victim is presented, including forehead flap reconstruction following complete nasal amputation.

CONCLUSION: Pediatric dog attacks are preventable injuries since the majority are from dogs defined as a dangerous breed. These cases are the "tip of the iceberg" representing consultations to Plastic Surgery only. We describe the scope of the problem, preventative guidelines, and outline why organizational advocacy in plastic surgery should be directed towards a national prohibition of dangerous dogs.

Learning Objectives:

- Characterize the nature of pediatric dog attack injuries, including patient demographics and operative interventions.

- Understand the implications of dangerous dog breeds on the pediatric population, including physical and psychological morbidity.

- Advocate for increased governmental intervention, including legislation and educational programs restricting dangerous dog breeds.

\section{6}

PLASTIC SURGERY TRAUMA ASSESSMENT RECORD: AN EVALUATION OF THE ENHANCEMENT OF MEDICAL EDUCATION AND IMPROVEMENT OF PATIENT CARE IN THE EMERGENCY ROOM

\section{A Seal, D Courtemanche, $K$ Bush}

PURPOSE: To investigate the usefulness of a standardized assessment record for the initial assessment of Plastic Surgery trauma patients in the emergency room. Specifically, the goals are to assess if medical students and junior residents have an improved educational experience using this standardized record and to evaluate if the record facilitates the comprehensive examination and documentation of these clinical encounters.

METHODS: Medical students and junior residents were randomized to either complete a standard consult or a new assessment record after assessing plastics surgery trauma patients in the ER. After review with a senior resident or staff, they completed a survey about their experience. The consults were also scored for content with respect to general history and physical exam, hand specific content, and craniofacial specific content where appropriate.

RESULTS: There were 31 consults reviewed, (17 standard, 14 new). Mean self-assessment scores were significantly different (standard $=32$, new $=39, p=0.002$ ). Subjective clinical assessment, documentation, and educational experience were significantly improved with the new assessment $(\mathrm{p}=0.008, \mathrm{p}=0.002$, and $\mathrm{p}=0.002$ respectively). There was no significant difference in the General Score between the two consults. The Hand and Craniofacial Specific Scores were both significantly improved with the new consult $(3.0+/-0.5$ vs $5.6+/-0.4, p=0.001$, and $3.9+/-0.9$ vs. $8.0+/-1.2, \mathrm{p}=0.02)$.

CONCLUSION: Medical students and junior residents rate their educational experience and overall clinical experience to be improved using the new assessment tool. There is no improvement in documentation of the general history and physical exam of plastic surgery patients seen in emergency, however, there is a significant improvement in the hand specific exam, as well as the craniofacial specific exam.

Learning Objectives:

At the end of this presentation, the learner will be able to:

- Assess the usefulness of standardized assessment records for plastic surgery trauma patients.

\section{7}

\section{TAKING THE RESIDENT BY THE SCALPEL: A PUBLIC SURVEY}

D Rizis, DE Borsuk, E Botros, JP Brutus

PURPOSE: An effective surgical training program ensures that all surgical residents have adequate exposure and training in surgical procedures. However, higher rates of nosocomial infections and increased public awareness of medical errors have recently created pressure to take the scalpel out of the residents' hands.

METHODS: In an effort to quantify and qualify public opinion and knowledge of the surgical education system, we have presented a questionnaire to all new patients consulting for hand surgery at a large urban teaching hospital in Montreal, Canada. The questionnaire determines age, socioeconomic status, and previous exposure to health care. It examines knowledge of the surgical hierarchy, and the public's willingness to have surgery done in-part, or in full, by a supervised surgical trainee.

RESULTS: 105 patients consulting for elective hand surgery completed the questionnaire. 19 (18\%) would not consent to have a surgical resident operate on them even with the supervision of the attending surgeon, citing fear of increased complication rate as the major reason for the refusal. However $97 \%$ of patients consented to having their photos or $\mathrm{x}$-rays used for training purposes. $85 \%$ of the patients were unable to properly differentiate between a clinical clerk, a resident, and an attending surgeon. After having read a short explanation of surgical hierarchy 4 patients (3.8\%) modified their opinions, consenting to surgery by a supervised a surgical resident. 
CONCLUSION: A good surgical training program ultimately guarantees that every surgeon produced has the skills and know-how to operate effectively and safely. Our public survey demonstrates that the public is generally willing to allow surgical residents to operate on them for hand surgeries if properly informed. However, almost one in five would not allow a trainee to operate on them in a university hospital. Education on the surgical hierarchy and operative diligence is vital for the future of surgical residencies.

Learning Objectives:

- To identify and qualify the public's lack of knowledge of medical hierarchy.

- To acknowledge the importance of informed consent in the teaching hospital setting.

38

\section{Canadian Expert Series}

\section{SC-IGAP FLAP BREAST RECONSTRUCTION}

\section{S Williamson}

PURPOSE: To discuss the clinical utility of the septocutaneous IGAP perforator flap in autogenous breast reconstruction.

METHOD: Clinical indications, flap design concepts, operative technique, functional and aesthetic outcome, and donor morbidity are reviewed in an effort to delineate the abilities and limitations of this flap for autogenous breast reconstruction.

RESULTS: Sc-IGAP breast reconstruction with the "in the crease technique" can be effective in achieving the appropriate patient's desired breast mound reconstruction with acceptable donor site morbidity. Donor site morbidity is increased with aggressive skin \&/or subcutaneous harvests. Immediate bilateral reconstructions are ideally suited, but the multiple intra-operative repositionings required lead to substantial operative times.

CONCLUSIONS: The septocutaneous IGAP perforator flap, while not widely described, is a viable "alternative flap" for autogenous breast reconstruction. Its attributes of ease of harvest, pedicle length, ability to neurotize, lack of muscular injury and acceptable donor deformity must be balanced with an appreciation of its physical attributes, potential donor morbidity and need for intra-operative repositioning in considering its indications for each individual patient.

Learning Objectives:

- To consider the attributes, limitations and utility of the septocutaneous IGAP perforator flap in autogenous breast reconstruction.

\section{9}

\section{THE USE OF ALLODERM IN POST-MASTECTOMY} ALLOPLASTIC BREAST RECONSTRUCTION: A SYSTEMATIC REVIEW AND ECONOMIC ANALYSIS

\section{LA Jansen, SA Macadam}

PURPOSE: To perform a systematic review on the use of AlloDerm in post-mastectomy alloplastic breast reconstruction, and an economic analysis comparing one-stage AlloDerm reconstructions with traditional two-stage reconstructions.

METHOD: A computerized search was performed across databases to identify studies including patients undergoing alloplastic breast reconstruction with AlloDerm. A systematic review was performed across study types, yielding probabilities for seven clinically significant health outcomes. A decision analytic model from the provincial payer's perspective was constructed based on these states. Direct medical costs were estimated from a university-based hospital, yielding expected costs. Sensitivity analyses were conducted.

RESULTS: The systematic review of studies using AlloDerm in breast reconstruction revealed 14 papers satisfying inclusion criteria ( 7 onestage, 6 two-stage, 1 including both). Acute and long-term complication rates were obtained. One-stage complication rates were: infection $0-11 \%$, hematoma $0 \%$, seroma $0-9 \%$, partial flap necrosis $0-25 \%$, implant exposure with removal $0-9 \%$ / salvage $0-4 \%$ and capsular contracture $0 \%$. No objective validated outcomes or cost analyses were reported. Baseline and expected costs were calculated for one-stage
AlloDerm and two-stage traditional reconstructions. Preliminary cost analysis shows one-stage reconstruction with AlloDerm to be less expensive both in the baseline $(\$ 11,676$ vs. $\$ 13,884)$ and expected value $(\$ 12,361$ vs. $\$ 14,825)$ if a $4 \times 16 \mathrm{~cm}$ sheet is used. With a $6 \times 16 \mathrm{~cm}$ sheet, the one-stage baseline and expected costs rise to $\$ 13,367$ and $\$ 14,053$ respectively.

CONCLUSIONS: Complications using AlloDerm are comparable to traditional alloplastic reconstructions. AlloDerm appears to confer a low rate of capsular contracture. Although AlloDerm is expensive, it appears to be cost effective if used for single stage reconstructions. A formal randomized control trial, including costs, is recommended.

Learning Objectives:

- Become familiar with the rationale and methodology for performing a systematic review.

- Apply results from a systematic review to perform a cost analysis.

\section{0}

\section{SINGLE STAGE PROSTHETIC BREAST RECONSTRUCTION AT TIME OF MASTECTOMY: A RETROSPECTIVE, MATCHED COHORT STUDY}

\section{A Chesney, F Farrokhyar, A Dal Cin}

PURPOSE: Immediate breast reconstruction in patients with early stage disease is gaining popularity across North America. With the advent of skin sparing mastectomy, immediate reconstruction with the definitive, final prosthesis at the time of mastectomy has been made possible. The question remains: is single-stage prosthetic reconstruction equivalent to tissue expander reconstruction with respect to complications and rate of reoperation?

METHODS: A retrospective, matched cohort study of a single centre's experience with these techniques was carried out. Over a five year period, 40 cases of immediate breast reconstruction with the final prosthesis were identified. These were matched to 40 cases of immediate breast reconstruction using a tissue expander. The matching process controlled for such factors as patient age, mastectomy weight, the presence of comorbidities and prior exposure to radiation. All patients had early stage disease or were undergoing prophylactic mastectomy. Complication rates as well as the rate of re-operation were examined. RESULTS: No significant increase in the rate of post-operative complications or rate of re-operation was found to be associated with the insertion of a definitive prosthesis at the time of skin sparing mastectomy. The average time to completion of the reconstructive process was zero months in the immediate prosthesis cohort and seven months in the tissue expander cohort.

CONCLUSIONS: These findings support that, in selected patients, single stage breast reconstruction using the final prosthesis is not associated with an increased risk of complications or reoperation. Furthermore, the average time to completion of reconstruction is reduced by seven months.

Learning Objectives:

- This study promotes an understanding of the technique of single-stage prosthetic breast reconstruction at the time of mastectomy. This technique is as safe as the use of expanders and reduces the time to completion of reconstruction.

\section{1}

BREAST RECONSTRUCTION AND RADIATION THERAPY: A CANADIAN PERSPECTIVE

O Afolabi, J Williams, DH Lalonde

PURPOSE: Radiation therapy is increasingly being used as an adjunctive treatment for breast cancer. When, and how best to perform breast reconstruction in the setting of radiation therapy is a much debated topic. The aim of this project was to investigate the approaches that Canadian plastic surgeons are taking to breast reconstruction in patients who need or may need radiation.

METHODS: In April 2009, a survey invitation was sent to Canadian plastic surgeons via email. Survey responses were collected over a two month period. 
RESULTS: Of the 307 invitees, 87 surgeons responded. Eleven did not meet inclusion criteria. Most surgeons (70\%) do not perform immediate reconstruction on patients who need post-mastectomy radiation. Most respondents (64\%) perform immediate reconstructions for patients whose need for radiation is uncertain at the time of mastectomy. Expander and implants is their preferred option, followed by free TRAM flap. Fifty five percent use the delayed immediate technique in these cases. Thirty-two percent are unfamiliar with the delayed-immediate technique.

For delayed reconstruction of the irradiated patient, the pedicled TRAM is the most common choice. The latissimus dorsi flap was the second most common choice.

Factors that affect surgeon decision making vary from the thickness and pliability of the patient's skin following radiation to the cooperation of colleagues such as general surgeons and radiation oncologists.

CONCLUSIONS: The reconstructive options are increasing for patients who may need post-mastectomy radiation. The use of the delayed immediate technique could increase as physicians gain more knowledge of the technique.

Learning Objectives:

- List the current indications for radiation therapy in patients requiring mastectomy for breast cancer.

- Describe the challenges of breast reconstruction in the irradiated patient.

- Explain the delayed-immediate technique of breast reconstruction and the indications for its use.

\section{2 \\ POST-MASTECTOMY IRRADIATION FOLLOWING IMMEDIATE ALLOPLASTIC BREAST RECONSTRUCTION USING A TEMPORARY TISSUE EXPANDER: A UBC PERSPECTIVE}

AL Ho, AD Malebranche, S Tyldesley, S Macadam, PA Lennox

PURPOSE: Radiation combined with mastectomy and chemotherapy is associated with a decrease in recurrence and improvement in survival rates in breast cancer patients. Thus, a greater number of patients who desire breast reconstruction will have radiation as part of their management. The timing of post-mastectomy radiation in alloplastic breast reconstruction patients is still a subject of debate. As part of the UBC Breast Reconstruction Program, breast cancer patients receive radiation at the time of maximal expansion prior to permanent implant exchange. We report our outcomes.

METHOD: We retrospectively reviewed breast cancer patients receiving immediate breast reconstruction using a two-stage expander/implant technique between 2003 to 2007. Data were drawn from patient records and the UBC Breast Reconstruction and BC Cancer Agency databases. RESULTS: Outcomes including capsular contracture, revision rates, and aesthetic outcome were compiled after retrospective review.

CONCLUSIONS: Findings were compared to the Memorial Sloan Kettering experience in which radiation is performed after implant exchange in patients undergoing immediate alloplastic breast reconstruction.

Learning Objectives:

At the end of this presentation, the learner will be able to:

- Appreciate the different treatment algorithms, with respect to postmastectomy radiation, of immediate tissue expander/implant breast reconstruction.

- Appreciate the challenges of radiation treatment for cancer patients receiving immediate breast reconstruction using a tissue expander/implant technique.

- Compare the clinical outcomes of the UBC Breast Reconstruction Program to the Memorial Sloan Kettering algorithm.

\section{3}

\section{LOCAL BREAST CANCER RECURRENCE AFTER MASTECTOMY AND IMMEDIATE RECONSTRUCTION FOR INVASIVE CANCER: A META-ANALYSIS}

\section{Dickson, R Avram, M Gieni, N Sne}

PURPOSE: There is a lack of consensus as to the oncologic safety of IBR, particularly in those with stage III or IV disease. The purpose of this paper is to systematically review the literature to and compare the frequency of recurrence in patients with and without IBR following mastectomy for first time, invasive breast cancer.

METHODS: Two independent investigators searched PubMed, Embase, and Cochrane databases using the following search terms: "immediate reconstruction" and "breast cancer", "mammary", "carcinoma". This returned a total of 3080 titles. After the titles were reviewed, 228 abstracts remained. The abstracts were reviewed and resulted in 143 relevant articles. After inclusion \& exclusion criteria were applied, 6 articles remained. Each article was assessed for quality by two independent investigators using a standardized scoring scale (MINORs). Relevant data were collected. The primary outcome was local recurrence of breast cancer.

RESULTS: Inter-rater reliability was $75 \%$ with $95 \%$ confidence intervals. There was no evidence of study heterogeneity $\left(\mathrm{I}^{2}=16 \%\right)$. The odds ratio for recurrence of breast cancer for mastectomy and IBR as compared to mastectomy alone was 0.832 , indicating no significant difference. Subgroup analysis was not performed for recurrence based on stage or reconstruction type due to lack of data reported.

CONCLUSION: This meta-analysis, while limited by the quality of the studies it reviewed, demonstrated no evidence of increased frequency of breast cancer recurrence when immediate post-mastectomy reconstruction is compared with mastectomy alone in patients with invasive breast cancer. Data on recurrence rates in immediately reconstructed patients with stage III \& IV disease is limited and future studies should include this patient population.

Learning Objectives:

- To appreciate the role of immediate breast reconstruction in breast cancer treatment and its oncologic safety.

- To recognize the importance of prospective outcome studies in determining the local recurrence rates in advanced stage breast cancer.

\section{4}

\section{PERIOPERATIVE FLUID CONSIDERATIONS TO MINIMIZE EARLY POSTOPERATIVE COMPLICATIONS IN FREE FLAP BREAST CANCER RECONSTRUCTION}

R Neinstein, T Zhong, S Hofer, JE Lipa, PC Neligan

PURPOSE: Breast reconstruction at the same time of mastectomy has proven to be a safe and reliable procedure that does not compromise oncologic outcomes. An important aspect of free flap breast reconstruction that has not been previously studied pertains to the perioperative anesthetic and fluid management of these patients. Thus the purpose of this study is to assess if there is an association between these factors with postoperative complications in patients undergoing free flap breast reconstruction.

METHOD: A retrospective review was performed for the period between 2002 and 2009. All consecutive patients with free flap reconstruction of the breast following mastectomy performed at University Health Network were identified in a prospectively maintained breast reconstruction database. Perioperative anesthetic variables analyzed included: BMI, ASA, preoperative hemoglobin, hematocrit, creatinine levels, and intraoperative colloid infusion rate/kg, crystalloid infusion rate $/ \mathrm{kg}$, and total fluid volume infusion rate $/ \mathrm{kg}$. Primary clinical outcomes included all minor and major post-operative complications. RESULTS: Univariate analysis revealed that both total volume of fluid infusion rate $/ \mathrm{kg}(\mathrm{p}=0.015)$ and crystalloid infusion rate $/ \mathrm{kg}(\mathrm{p}=0.013)$ during the surgery were significant independent risk factors for postoperative complications. Our final multivariate regression model showed that after controlling for the type of flap used (DIEP vs. free TRAM vs. other flaps) and effect of other covariates such as presence of a cardiac 
risk factor, both BMI and amount of crystalloid infused/kg were all significantly related to postoperative complications $(\mathrm{p}=0.0448$ and 0.0207 respectively). Furthermore, we found that a statistically significant quadratic relationship existed between the rate of peri-operative fluid infusion and risk of complication. Outside of the middle range of infusion rates, on either the low or high ends, higher risk of complication was observed.

CONCLUSIONS: This is the first study to show that perioperative fluid management is related to post-operative complications in breast free flap surgery.

Learning Objectives:

- Participants should appreciate what is currently known in the surgical literature about the predictors of morbidity in breast cancer free flap reconstruction.

- Participants should appreciate that perioperative fluid management may be a modifiable predictor of morbidity in breast cancer free flap reconstruction.

\section{5 \\ REDUCED LENGTH OF STAY IN HOSPITAL FOR BURN PATIENTS FOLLOWING A CHANGE IN PRACTICE GUIDELINES}

$\underline{\text { S Hynes, A Papp }}$

PURPOSE: A change in burn care practice guidelines was initiated at an adult provincial burn centre in support of more aggressive excision and grafting procedures and new burn dressing protocols. The current study is an initiative to assess the outcomes of burn patients before and after these changes were implemented.

METHOD: Data were retrospectively extracted from the study centre's burn registry database. Demographics, mean number of procedures per admission, and mean time to first procedure were recorded. Outcomes were determined by length of stay, readmission rates, complications (e.g. infections, ACS, PE/DVT), transfusion requirements, and mortality. A comparison was made between patients admitted from August 1, 2005 to July 31, 2007 and those admitted from August 1, 2007 to July 31, 2009.

RESULTS: The 2 groups ( $\mathrm{N}=164$ and 181 patients) were confirmed to be comparable with respect to age $(41.5 \pm 9.1$ vs. $38.4 \pm 18.0, p=0.131)$ and $\%$ TBSA $(13.3 \pm 13.3$ vs. $13.1 \pm 14.7, \mathrm{p}=0.898)$. There was a higher incidence of inhalation injury in the earlier group $(27.4 \%$ vs. $9.4 \%$, $\mathrm{p}=0.00001$ ). There was a significant difference in mean length of stay $(24.4 \pm 40.9$ vs. $15.4 \pm 19.7, \mathrm{p}=0.011)$, as well as mean length of stay/\%TBSA from 2.4 to 1.6 days. There was no difference in mortality between the 2 groups $(4.9 \%$ vs. $6.1 \%, p=0.626)$. The rate of readmission, complications, and transfusion requirements are still to be determined.

CONCLUSIONS: The $33 \%$ reduction in length of stay since the implementation of new practice guidelines could represent a major advancement in burn care and burn resource utilization for the study centre. The apparent difference in the incidence of inhalation injury between the groups is likely due to an increase in bronchoscopic confirmation of inhalation injury over the past 2 years. A chart review and subgroup analysis will be carried out to further elucidate the reasons for this reduced length of stay.

Learning Objective:

- The audience will gain insight into whether a more aggressive operative approach to burns (higher \%TBSA excised and grafted per procedure) is a safe and effective way to reduce length of stay in hospital.

\section{6}

\section{MEASURING THE UTILITY OF THE BURNED FACE FROM THE GENERAL PUBLIC AND EXPERT PERSPECTIVE} J Chuback, A Thoma, S Martin, A Yarascavitch

PURPOSE: Facial composite allotransplantation (FCTA) is a potential reconstructive option for severe facial disfigurement. With the use of health utility methods, a previous study has shown that a significant gain in valuation of quality of life (as measured by quality adjusted life years (QALYs)), may occur with FCTA. However, this study was conducted on a limited population. In order to extrapolate earlier study results, we conducted the decision analysis model in the general population and amongst burn professionals.

METHODS: The potential complications associated with facial burn scar and facial CTA were identified by a review of burn, kidney and hand transplant literature. Utilities of facial burn scar and FCTA were obtained from the general public and burn professionals, using the standard gamble (SG) and time trade-off (TTO) measures. The utilities were then translated into QALYs. QALY gained were calculated and tested for robustness using a sensitivity analysis.

RESULTS: Amongst the public $(\mathrm{N}=27)$ mean QALY calculated by TTO was 7.8 years, and decreased to -9.8 years according to the sensitivity analysis. QALY calculated by SG was 13.7 years, and decreased to -3.6 years according to the sensitivity analysis.

Amongst professionals $(\mathrm{N}=24)$ mean QALY calculated by TTO was 12.3 years, and decreased to -2.3 years according to the sensitivity analysis. QALY calculated by SG was 12.7 years, and decreased to -1.5 years according to the sensitivity analysis.

CONCLUSIONS: Ongoing debate over the potential benefit of FCTA can be assisted with health utility analysis. Knowledge of the QALY associated with FCTA allows for comparison against other health interventions.

Learning Objectives:

- To appreciate health utilities, and the methods by which they can be obtained.

- To appreciate how health utility data applies to plastic surgical innovation.

\section{7}

\section{SUCCESSFUL UTILIZATION OF ARTIFICIAL SKIN SUBSTITUTE (INTEGRA®) IN SOFT TISSUE RECONSTRUCTION}

\section{A Al-Kudmani, M Gomez, J Fish}

PURPOSE: To determine the outcome of soft tissue surgical reconstruction procedures with the use of artificial skin substitute (Integra $\left.{ }^{\circledR}\right)$.

BACKGROUND: Artificial Skin Substitute (Integra $\left.{ }^{\circledR}\right)$ is an acellular permanent dermal template that consists of outer silicone layer (polysiloxane polymer) and an inner bovine tendon collagen type-1 with chondroitin-6-sulfate (shark cartilage GAG).

It was first introduced in 1981 by Burke and Yannas, after a randomized controlled trial by Heimbach et al in 106 patients with major burns. Integra ${ }^{\circledR}$ was approved in March 1996 by the U.S Food and Drug Administration for use in acute burn injuries. Then, the application of Integra ${ }^{\circledR}$ in acute burn injuries became widely and extensively acceptable, but its use in soft tissue reconstruction despite been approved is not widely applied.

METHOD: The authors present a series of 10 patients who underwent soft tissue reconstruction with the use of Integra ${ }^{\circledR}$ between January 1 , 2000 and October 31, 2008. Retrospective chart review and a patient satisfaction survey were used to measure the success of the use of Integra ${ }^{\circledR}$ in terms of bone coverage, scar assessment, and patient satisfaction.

The data were obtained 2-10 years following the reconstruction.

The Student's $t$-test was used for continuous variables (age, length of hospital stay, operation time, etc.), and the $\chi^{2}$ analysis was used for non-parametric variables (gender, complications, patient satisfaction), with a $\mathrm{p}<0.05$ considered significant

RESULTS: There were 8 males and 2 females with a mean age of $47.5 \pm 13.3$ years. The majority of patients had work-related injuries $(90 \%)$, and the cause of soft tissue defects were burns (80\%), or skin cancer (20\%). Five of the soft tissue reconstructive procedures were in the upper extremity, five in the lower extremity, and one in the head. Eight out of 10 patients had adequate bone coverage.

Nine out of 10 patients were satisfied with their soft tissue reconstruction using Integra ${ }^{\circledR}$ in relation to bone coverage, rate of complications and reoperation, scar quality, sensitivity, sweating, color and texture match. One patient had hematoma and two patients had loss of 
Integra ${ }^{\circledR}$ over exposed bone, which required other means of soft tissue coverage.

CONCLUSION: The use of Integra ${ }^{\circledR}$ is a valuable option for soft tissue surgical reconstruction with satisfactory results without burning any bridges in a complex soft tissue defects.

Learning Objectives:

- At the end of this lecture, the learner will be able to define Integra ${ }^{\circledR}$, mechanism of action, indications, contraindications, uses and possible complications.

- Participants will be able to identify the prerequisites for the successful use of Integra® in complex soft tissue defects.

- Participants will be able to consider the use of Integra® in soft tissue defects (without burning bridges) that otherwise will need major reconstructive options.

\section{8}

\section{HALIFAX PRESSURE SORE PREVENTION STUDY}

\section{K Calder, B Chung , C Bebbington, A Murphy, J Paletz, J Williams}

PURPOSE: Pressure sore management is the most common reason for inpatient plastic surgery consultation at our institution. The goal of this study is to identify the risk factors predisposing patients to pressure sore formation and to evaluate the adherence to prevention strategies currently in place at the Queen Elizabeth II: Health Sciences Centre.

METHODS: Patients with the International Classification of Diseases (ICD-10-CA) listing L89: "Decubitus ulcer" were identified using the Capital District Health Authority (CDHA) Health Information Services coding system for the years 2007-2008. Each patient chart was reviewed to assess adherence to the hospital policies and procedures manual regarding the use of the Braden Scale Risk Assessment Tool. Individual category scores as well as composite scores were recorded. In addition patient demographics, pressure sore characteristics, the use of pressure reduction surfaces and multidisciplinary consultations were assessed.

RESULTS: 196 patients were diagnosed with a pressure sore(s). 68 patients (35\%) developed a pressure sore during hospital admission. Only 20\% of patients had a Braden scale assessment on admission [median score $=15$ (range: $9-21)$ ]. $15 \%$ patients had a pressure reduction surface prior to ulcer diagnosis. Ulcer grades were I-(10\%), 2-(52\%), 3-(24\%), and 4-(14\%). The most common ulcer locations were the coccyx (76\%), and heel (19\%). Pressure sore development as an inpatient was associated with a $20 \%$ mortality.

CONCLUSIONS: The current prevention strategies in place at our hospital are inadequate in preventing pressure sore formation. Interventions are required to modify patient risk factors and support adherence to CDHA policies. We recommend a standard pre-printed order form be implemented on admission for at risk patients to facilitate a preventative multidisciplinary approach.

Learning Objectives:

- Participants will learn and understand the variables associated with the development of pressure sores in hospital inpatients and will be able to identify interventions required for pressure sore prevention.

\section{9}

\section{THE QUALITY OF REPORTING OF META-ANALYSES IN PLASTIC AND RECONSTRUCTIVE SURGERY OVER THE PAST THIRTY YEARS}

\section{S Chan, $L$ Jansen, $M$ Bhandari}

PURPOSE: Meta-analyses of high quality studies represent Level I evidence and have important impacts on health care delivery. However, not all published meta-analyses are rigorously performed or reported. The purpose of this study was to evaluate the quality and the quality of reporting of meta-analyses over a 30-year span in a high impact plastic surgery journal.

METHOD: Two reviewers independently searched MEDLINE and EMBASE (January 1, 1980 - February 12010 ) and conducted a hand search to identify meta-analyses published in Plastic and Reconstructive Surgery. Predetermined inclusion criteria were applied to each identified study independently by two authors. Quality of the included meta-analyses was assessed in duplicate using the validated Overview of Quality Assessment Questionnaire (scored 1-7, 7=high quality; OQAQ). Quality of reporting was assessed using the well established QUORUM checklist and the more recently published PRISMA checklist.

RESULTS: Of 17,296 articles published in the journal over the 30-year span, 16 were meta-analyses. The overall quality of meta-analyses was found to be poor, with an estimated mean OQAQ score of 3.0 (95\% CI 1.4-4.6). We noted specific aspects of reporting to be particularly poor, such as assessing bias within and across studies. The new PRISMA statement expands upon the QUORUM and provides more detail regarding shortcomings in reporting.

CONCLUSIONS: Meta-analyses published in Plastic and Reconstructive Surgery have important limitations in reporting quality. Their results should be interpreted cautiously. Opportunities to improve reporting may require enforcement of published reporting guidelines, such as PRISMA, to ensure transparency of methodology.

Learning Objectives:

- To explain the role of meta-analysis in the hierarchy of evidence.

- To ensure a fundamental understanding of meta-analysis checklists.

- To explore the quality of meta-analyses in plastic surgery and opportunities for improvement.

\section{0}

STRATEGIES FOR HANDLING TREATMENT SELECTION BIAS IN NON-RANDOMIZED SURGICAL STUDIES:

A FUNCTIONAL OUTCOMES ANALYSIS OF SOFT TISSUE
SARCOMA PATIENTS UNDERGOING EXTREMITY SALVAGE
WITH AND WITHOUT FLAP RECONSTRUCTION
WITH AND WITHOUT FLAP RECONSTRUCTION

K Davidge, J Lipa, G Tomlinson, R Wong, J Wunder, A Davis

PURPOSE: Observational studies examining the impact of surgical interventions on patient outcomes have many methodologic challenges. This study sought to compare three strategies for handling selection bias in a functional outcomes analysis of extremity soft tissue sarcoma (ESTS) patients undergoing limb salvage surgery.

METHODS: A retrospective cohort study was conducted to evaluate the impact of flap reconstruction on postoperative function in ESTS patients. Patients treated with and without flap reconstruction were compared on three dimensions of function (impairments, activity limitations, and participation restrictions) using three methods to adjust for confounding: multivariable regression, matched sampling, and propensity score stratification. Propensity scores represented patients' probability of receiving a flap for limb salvage.

RESULTS: Patients in the flap $(n=56)$ and primary closure $(n=191)$ groups differed on several baseline characteristics (e.g. tumor size). Unadjusted analyses demonstrated more postoperative impairments $(\mathrm{p}=0.004)$ and activity limitations $(\mathrm{p}=0.013)$ in the flap reconstruction group. Adjusted analyses using regression, matching and propensity scores were consistent and did not demonstrate a significant effect of flap reconstruction on functional outcomes. Multivariable regression adjusted for multiple confounders, but adequacy of adjustment could not be ascertained. Case-control matching was limited to two key variables to preserve adequate sample size; baseline differences thus persisted within the matched cohort ( $n=45$ pairs). Stratification of patients into propensity score quintiles clearly demonstrated, but did not perfectly balance, the distribution of confounders between study groups.

CONCLUSION: Type of wound closure was not a significant determinant of postoperative function in ESTS, irrespective of how confounding variables were handled. However, regression, matching, and propensity-based stratification differed in their ability to demonstrate and adjust for confounding, and in their ease of application and interpretation.

Learning Objectives:

- To understand different methods for handling selection bias in observational studies.

- To understand the impact of flap reconstruction on functional outcomes in ESTS. 
51

\section{BURNOUT AND CAREER SATISFACTION AMONG RESIDENTS OF PLASTIC AND RECONSTRUCTIVE SURGERY ACROSS CANADA}

K Wanzel, $\underline{T}$ Alrasheed

PURPOSE: This study was undertaken to determine the incidence of burnout among plastic surgery residents across Canada and to identify the factors that are associated with burnout development to minimize burnout and improve the quality of residency education.

STUDY DESIGN: A cross-sectional questionnaire-based study of 120 plastic and reconstructive surgery residents across Canada was performed.

METHOD: A confidential questionnaire was mailed to all 120 plastic surgery residents across Canada in January 2010 and again in February 2010. The questionnaire consisted of five parts assessing the following elements: 1) demographic information, 2) professional stressors, 3) personal and professional life satisfaction, 5) symptoms of depression using the 2-item Primary Care Evaluation of Mental Disorders (PRIME MD) and 6) Maslach Burnout Inventory- Human Services Study.

RESULTS: The response rate was 40\% (48/120). Current first-year through fifth-year residents were included. The residents were on average 30 years of age. The MBI-HSS scores demonstrate 3\% of residents experiencing high burnout, $81 \%$ of residents with moderate burnout, and $16 \%$ of residents with low burnout. On average, residents have low depersonalization scores, low-moderate emotional exhaustion scores, and low-moderate personal accomplishment scores. Large amount of information to learn was the only burnout factor associated with all three burnout scales, 11 other burnout factors were associated with at least two burnout scales. Career satisfaction was negatively associated with burnout.

CONCLUSION: Most plastic and reconstructive surgery residents across Canada experience moderate levels of burnout. The strongest predictors of burnout were large amount of information to learn during residency, inadequate research tome, and having no time for extracurricular activities. Modification of risk factors should be undertaken to curb the development of burnout and its deleterious sequelae.

Learning Objectives:

- At the end of this presentation, the learner will be able to list the three dimensions of burnout, to identify the factors associated with the development of burnout and determine the modifiable factors that will minimize burnout and improve the quality of residency education.

\section{2}

\section{Invited Guest Lecture}

\section{RHINOPLASTY: SOME USEFUL POINTS}

\section{Bahman Guyuron, MD}

Rhinoplasty remains one of the most challenging procedures in plastic surgery. Those who are committed to learning and teaching rhinoplasty will continue to search for an ideal technique for perpetuity. The number of potential interplays during rhinoplasty makes achieving consistent, ideal results elusive. In this presentation, the author will share the evolution of his technique over the last 24 years. The author will elaborate upon those techniques that he finds more predictable and that commonly provide a natural appearing nose. Emphasis will be placed on specific important points of facial analysis, preoperative difficulties, intraoperative problems, and surgical techniques available to overcome the unnatural and "operated" rhinoplasty outcomes. The importance as well as the means of achieving proper dorsal outlines, a congruent tip and dorsal relationship, adjustment of tip width abnormalities, correction of alar and columella disproportions, and improvement in nostril morphology will be expounded upon.

Learning Objectives:

At the end of this course you will be able to:

- Define the techniques that are the most predictable and commonly provide a natural appearing nose.

- Recognize the importance of facial analysis.
- Formulate the pre-operative difficulties.

- Identify the potential intraoperative problems.

- Implement surgical techniques that are available to overcome the unnatural and "operated" rhinoplasty outcome.

Disclosure of Interest:

Dr. Guyuron has noted the following:

- He is a shareholder in Noteworthy Electronic Medical Record and owner of Innovative Medical Equipment.

- He is editor of Plastic Surgery Indications and Practice.

General Session

53

Canadian Expert Series

ORBITAL FRACTURES: RECENT ADVANCES AND CURRENT MANAGEMENT

\section{O Antonyshyn}

Orbital fractures are common injuries which can potentially lead to significant deformity and functional impairment. Accurate diagnosis and effective primary reconstruction of the disrupted orbit are the two major factors which can affect a favorable outcome.

Recent advances in imaging technology, surgical approaches and in intraoperative visualization have dramatically improved the surgeon's ability to achieve these goals.

The presentation summarizes our current management of periorbital blowout fractures and highlights the clinical application of these new technologies. Specifically, this presentation will describe:

1 . The role of multiplanar image rendering and interactive manipulation of image data.

2. The use of various eyelid incisions and ancillary approaches to maximize intraorbital visualization.

3. The applications of intraoperative 3D navigation.

4. The role of endoscopy in orbital surgery.

5. The choice of reconstructive materials and the reconstruction of orbital defects.

Learning Objectives:

- Diagnostic imaging techniques in the assessment of orbital trauma.

- Surgical approaches to the orbit.

- The application of intraoperative navigation and endoscopy in optimizing intraorbital visualization.

Disclosure of Interest:

Dr. Antonyshyn has noted the following:

- He is a member of the Advanced Cranio Maxillofacial Advisory Board (ACMF) North America.

- He has received an AO CMF Clinical Priority Program research grant.

\section{4}

PRE-OPERATIVE DIPLOPIA: THE MOST IMPORTANT PROGNOSTIC FACTOR FOR DIPLOPIA FOLLOWING SURGICAL REPAIR PURE ORBITAL BLOWOUT FRACTURE

Y Tahiri, LLee, M Tahiri, H Sinno, BH Williams, L Lessard, MS Gilardino

BACKGROUND: No consensus exists in the literature regarding the risk factors associated with new or residual diplopia after pure orbital blowout fracture (BOF) repair.

OBJECTIVE: To assess and evaluate the risk factors associated with diplopia after surgical repair of pure BOF.

METHODS: Patients with pure BOF who were managed surgically were identified in an 11-year period at the McGill University Health Center. The association between new or residual diplopia post-surgical repair and various risk factors were assessed using $\chi^{2}$ tests / Fischer's exact tests and multivariate analysis was conducted using logistic regression.

RESULTS: 61 patient charts were reviewed. Results demonstrated the presence of pre-op diplopia and radiological evidence of extra-ocular 
muscle (EOM) swelling to be strongly associated with diplopia at 6 months post repair $(\mathrm{p}<0.05)$. Patients who presented pre-operative with diplopia had a 9.91 times greater probability of developing diplopia post-operatively ( $p=0.035,95 \% \mathrm{CI}: 1.17-83.80$ ).

CONCLUSION: Pre-operative diplopia is the best predictor of the presence of post-operative diplopia following BOF repair. Initial injury to the EOM leading to EOM swelling and pre-operative diplopia seems to be at the origin of diplopia after surgical repair of pure BOF.

Learning Objectives:

- Evaluate the risk factors associated with diplopia after surgical repair of BOF. Review of the literature. Reach a consensus as to factor(s) associated with diplopia following surgical repair of BOF.

\section{5}

\section{A NEW SYSTEM FOR SEVERITY SCORING OF FACIAL FRACTURES: DEVELOPMENT AND VALIDATION J Catapano, JA Fialkov, PA Binhammer, C McMillan, OM Antonyshyn}

PURPOSE: While several widely accepted classification systems exist for facial fractures, these are mostly region specific and differ in the classification criteria used, making it impossible to uniformly and comprehensively document facial fracture patterns. Furthermore, a widely accepted system that is able to provide a final summary measure of fracture severity does not exist, making it difficult to investigate the epidemiologic data surrounding facial fracture severity. In this study, a comprehensive method for panfacial fracture documentation and severity measurement is proposed and validated.

METHOD: Through retrospective analysis, facial fracture pattern and severity were documented and calculated using the proposed scoring system for a population of 63 patients operated on for acute facial fracture. Intra- and inter-observer reliability were examined, and the severity scale was then validated through statistical analysis of correlation with surrogate markers of severity (number of implants and operating room procedure time).

RESULTS: Spearman correlation coefficients were calculated and a statistically significant correlation was found between severity score and both number of implants and operating room procedure time $(\mathrm{R}=$ 0.92790 and $R=0.68157$ respectively). Intra-class correlation coefficients were calculated to assess intra-rater and inter-rater reliability of the severity scale and were found to be high (0.97 and 0.99 respectively).

CONCLUSIONS: In this study, we have validated a novel severity score as a reliable representation of facial fracture severity and have demonstrated it as a practical instrument for the investigation of the epidemiological data surrounding facial fracture severity.

Learning Objectives:

This presentation will:

- Review current facial trauma classification systems.

- Introduce a new method of documenting facial fracture patterns.

- Demonstrate the use of this classification system in the objective evaluation of facial fracture outcomes.

\section{6}

\section{RESULTS OF PRIMARY ORBITAL FRACTURE REPAIR: AN ANALYSIS OF MORPHOLOGICAL AND AESTHETIC OUTCOMES}

\section{R Hontscharuk, JA Fialkov, CR McMillan, PA Binhammer,}

O Antonyshyn

PURPOSE: To develop, validate and implement a technique for evaluating outcomes of orbital reconstruction. Specific objectives:

To determine the degree of periorbital asymmetry in a normal population.

To evaluate the morphological results of primary orbital reconstruction in a pilot series of patients.

METHODS: Facial 3D images were captured using a Vectra 3D. Morphometric analysis was based on interactive identification of anthropometric landmarks and surface features. Validity was determined by quantifying the inter-rater variability among three raters using the Winer reliability coefficient.

The morphometric analysis was applied to a population of healthy adults $(n=13)$, and a population of patients following primary repair of unilateral orbital fractures $(n=13)$. All subjects received the Derriford Appearance Questionnaire (DAS59) to quantify facial appearancerelated quality of life.

Normative periorbital asymmetry was determined using the mean asymmetries and $95 \%$ confidence intervals in the healthy controls. The morphological results of primary orbital reconstruction were evaluated by identifying residual asymmetries, and comparing them to the control population using independent sample t-tests.

RESULTS: Normative periorbital asymmetry was established in a reference population with no previous orbital injury or surgery. The mean asymmetry was less than $1.6 \mathrm{~mm}$ for each parameter.

Restoration of normal symmetry following primary unilateral orbital fracture repair was achieved for 16 of 20 measured parameters. When compared to controls, the fracture population showed no significant differences in the degree of asymmetry in globe projection, lower eyelid position or length.

CONCLUSIONS: This study establishes a normative reference of periorbital asymmetry and analyzes the effectiveness of primary orbital reconstruction in restoring periorbital morphology.

Learning Objectives:

Participants will:

- Become familiar with normal periorbital morphology.

- Gain knowledge of outcomes following orbital fracture repair.

\section{7}

\section{FIVE-SEGMENT MANDIBULAR OSTEOTOMY: A NOVEL APPROACH TO THE CORRECTION OF COMPLEX POST-TRAUMATIC MANDIBULAR DEFORMITIES}

\section{Wong, S Smith, A Rideout, O Antonyshyn}

PURPOSE: Condylar neck fractures in combination with parasymphyseal or symphyseal fractures produce a highly unstable mandibular fracture pattern. Failure to achieve a stable reconstruction results in an established mandibular deformity characterized by increased bigonial facial width, decreased posterior facial height and malocclusion. The purpose of the study is to describe and evaluate a novel and effective surgical approach for correction of this complex post-traumatic mandibular deformity.

METHODS: The study series consisted of 5 consecutive patients presenting to Sunnybrook Health Sciences Centre between 1994 and 2007 with established malunion following combined condylar neck and parasymphyseal fractures, treated secondarily with a five-segment mandibular osteotomy.

All patients underwent preoperative orthodontics followed by a 5 part mandibular osteotomy comprising bilateral sagittal splitting, symphyseal and genioplasty osteotomies.

Data collected included clinical presentation, initial fracture pattern, and initial management. Results of treatment were evaluated in terms of change in occlusion, jaw excursion and facial appearance. Changes in facial morphology were objectively assessed based on morphometric evaluation of pre and post-op photographs.

RESULTS: Post-osteotomy, bigonial width, facial height and retrognathia were consistently improved. The ratios of bizygomatic to bigonial width and upper facial height to lower facial height increased. Four of the five patients showed no change in chin displacement relative to the facial plane. Malocclusion was corrected in all 5 patients, but partial relapse occurred in one case. No complications were reported.

CONCLUSION: Five-segment mandibular osteotomy is a safe and effective technique to correct complex mandibular deformities by re-establishing posterior mandibular height and mandibular arch width.

Learning Objectives:

- Participants will be able to list features of a post-traumatic mandibular deformity.

- Participants will be able to describe a surgical technique to correct posttraumatic mandibular deformities. 
58

\section{CT SCANS AND NON-SYNDROMIC SINGLE SUTURE CRANIOSYNOSTOSIS: INCIDENTAL FINDINGS AND ROLE IN MANAGEMENT}

\section{T Samson, N Gjoska, G Ben David, B Rechner, CR Forrest}

PURPOSE: Controversy exists regarding the use of CT scans in the management of non-syndromic, single suture craniosynostosis due to concerns related to the safety of medical radiation. While most surgeons agree that CT scans are unnecessary for diagnostic purposes, we contend that the risk benefit ratio favors the routine use of CT scan in the management of these infants. Our aim is to evaluate the usefulness of CT scan imaging in patients with single suture craniosynostosis.

METHODS: 195 children (134M; 61F) with a mean age of 11.5 months (3 to 108 months) presented for primary surgical management of single suture, non-syndromic craniosynostosis (105 sagittal, unicoronal 49, metopic 32, bicoronal 5, lambdoid 2, other 2) from 1999 to 2008. All surgeries were performed uneventfully with no mortalities or long-term morbidities. CT scans were performed before surgery, immediately postoperatively and 1 year following surgery. CT protocol included scout views followed by 1 sec helical 2.5 slice thickness @3.75 speed scan mode 0.75:1 120KVP $20 \mathrm{~mA}$ standard algorithm DFOV 22. A review of dictated reports was carried out and abnormalities were tabulated.

RESULTS: 652 CT scans were performed and reviewed. $19 \%$ of pre-op, $71 \%$ of post-op and $48 \%$ of 1 -year post-op scans demonstrated incidental/abnormal findings in addition to craniosynostosis for a total of 305/652 (47\%). Specific findings, radiation dose and impact on care will be discussed.

CONCLUSIONS: This study demonstrates that routine CT imaging identified unexpected abnormalities in a substantial group of patients. While CT scan imaging may be unnecessary for the experienced surgeon in diagnosis and surgical planning, a growing trend to avoid imaging these patients may result in rare but significant events impacting patient care.

Learning Objectives:

- To become familiar with the indications of CT scans in the management of single suture craniosynostosis.

\section{9}

\section{NON-OPERATIVE TREATMENT OF PIPJ FLEXION CONTRACTURES IN DUPUYTREN'S DISEASE}

\section{J Davidson, J Larocerie-Salgado}

PURPOSE: Proximal interphalangeal joint (PIPJ) contracture due to $\mathrm{DD}$ is considered by some to be an absolute indication for surgical intervention. However, post surgical outcomes can be inconsistent and are often associated with protracted rehabilitation, reduced flexion, recurrence of the contracture, and patient dissatisfaction. An alternative treatment option, comprised of splinting and passive stretching protocols, was introduced to stabilize early contractures of the PIPJ in the hopes of delaying or obviating surgery.

METHODS: Patients who primarily manifested mild to moderate PIPJ flexion contracture (between 15 and $55^{\circ}$ ) of the long, ring and/or little fingers were included in this trial. Patients were provided with a nighttime hand-based volar extension splint, stretching exercises into extension, and friction massage for PIPJ contractures associated with DD.

RESULTS: Over the course of approximately 11.5 months $( \pm 4.9)$, nine patients, in a total 11 fingers, were enrolled in this alternative treatment. Patients were 69 years old ( \pm 5.4 years). There were six males and four female patients. A total of eleven PIPJ, with flexion contractures, demonstrated extension ranging from $55^{\circ}$ to $20^{\circ}$. Overall, patients showed a significant improvement in active PIPJ extension of approximately $12.7^{\circ}$ (SD: $\pm 7.8^{\circ}$, range: $0-20^{\circ}$ ) over the course of the treatment ( $p=0.0003$ ); nine fingers demonstrated a general improvement of approximately $15.5^{\circ}$; two fingers revealed no significant changes in PIPJ extension.

CONCLUSIONS: Nighttime static extension splinting and soft tissue mobilization techniques, including massage and stretching exercises, appear to have a beneficial effect on flexion contractures of the PIPJ due to DD. Early non-operative intervention of PIPJ flexion contractures due to DD, including standard hand therapy protocols for soft tissue mobilization, may delay and possibly prevent the need for surgery.
Learning Objectives:

- Consider non surgical strategies in the management of DD.

- Understand the role of splinting in DD outside of post surgical rehabilitation.

60 Canadian Expert Series

\section{BREAST AESTHETICS AND SURGICAL PRINCIPLES}

\section{EJ Hall-Findlay}

The best way to understand surgery of the breast is to first understand the three dimensions of the breast and how each dimension can or cannot be altered surgically. The breast is a subcutaneous structure which is not attached to the chest wall but is held in place by skinfascial attachments at the inferior breast border (inframammary fold) and the medial breast border over the sternum. The upper breast border and lateral breast border are relatively free-floating. The third breast dimension consists of the shape of the breast that sits on the breast base or footprint. It is important to understand how to evaluate that shape and to be able to appreciate what is normal and what can be changed.

Learning Objectives:

- At the end of the session the student should be able to understand all three breast dimensions and describe what is normal. The student should be able to describe surgical maneuvers which can be used to change each of the four boundaries of the breast footprint - the upper breast border, the medial breast border, the lateral breast border and the inferior breast border (inframammary fold). The student should be able to describe the variations of the breast shape (third dimension) which are normal and which abnormalities can be addressed surgically.

\section{1}

MAKING THE CASE OF FAT GRAFT IN THE BREAST

AM Danino, E Botros, IGA Mojalal, F Arsenault

BACKGROUND: Several trials recently exposed the benefits of fat grafting in post cancer breast reconstructions or augmentation.

OBJECTIVE: Tried to define the Clinical Practice guideline for this surgical practice.

METHODS: A literature search was performed, using a defined search strategy, to identify the available publications relating to the specific topic. Following the selection and critical appraisal of the articles, the working group developed a document with a proposed guidelines based on scientific evidence and/or expert agreement. Recommendations are based on evidence syntheses, and explicit and transparent expert judgment.

Data Sources Medline, from 1953 to September 2009.

Level Definition

Level A: High standard meta-analysis or several high qualities randomized clinical trials that provide consistent results.

Level B: Good quality evidence issued from randomized trials (B1) or prospective or retrospective studies (B2). The results are consistent when considered together.

Level C: The methodology of the available studies is weak or their results are not consistent when considered together.

Level D: Either scientific data does not exist or there is only series of cases.

RESULTS: Only studies with level of evidence $\mathrm{C}$ or D conclude to the safety of fat grafting in terms of radiological follow up and tumor recurrence in case of residual breast tissues in the envelope, strong evidence of level A or B can support the potential risk of adipose grafting in contact with breast tissues.

CONCLUSIONS: This review suggest that fat grafting cannot be considered as a standard, an option or a recommendation. The recommendation consists in including patients within clinical trials only for breast reconstruction after complete mastectomy.

Learning Objectives:

- Systematic classification of the literature on fat graft.

- Red flags for plastic surgeons about breast fat graft. 
62

\section{SUBCUTANEOUS PRE-EXPANSION OF MASTECTOMY FLAPS PRIOR TO BREAST RECONSTRUCTION WITH DIEP FLAPS - ELIMINATING THE PATCH-LIKE APPEARANCE AND IMPROVING AESTHETIC OUTCOMES}

\section{K Schwarz, Y Tahiri}

INTRODUCTION: Free tissue transfer and tissue expansion are important tools in the reconstructive breast surgeon's armamentarium, yet are not often used in conjunction. While free tissue transfer has its advantages, the patch-like appearance of the skin paddle on the breast can be unappealing.

OBJECTIVE: To present our clinical experience of using subcutaneous breast tissue expansion prior to reconstruction with Deep Inferior Epigastric Perforator (DIEP) flaps, and show how this technique eliminates the patch-like appearance of the skin paddle, leaving only the native breast skin overlying the buried DIEP flap.

METHODS: Six patients underwent breast reconstruction using a threestage approach. During the first stage, tissue expanders were placed in the subcutaneous plane beneath the mastectomy flaps. Following complete tissue expansion, the second stage involved removal of the tissue expanders and reconstruction of the breasts by burying de-epithelialized DIEP flaps beneath the pre-expanded skin flaps. Revisions and nipple reconstructions were carried out in the third stage. Retrospective analysis of patients' characteristics, breast history, surgical stay, complications and outcomes were performed.

RESULTS: The patients were on average 49 years of age, with an average BMI of 25.7. Two of the patients underwent bilateral breast reconstruction while the rest had unilateral reconstructions. One patient, a smoker, required the evacuation of a seroma from the reconstructed breast as well as the treatment of a small donor site dehiscence. There were no DIEP failures or take-backs.

CONCLUSION: Using subcutaneous breast tissue expansion followed by DIEP flap reconstruction can be performed safely, offering patients a completely autologous breast reconstruction with low morbidity, as well as eliminating the classical patch-like appearance of flap reconstructions. Unlike traditional breast tissue expansion, the subcutaneous placement of the tissue expanders avoids the drawbacks associated with raising the pectoralis major prior to autologous reconstruction.

Learning Objectives:

- To present a new approach to breast reconstruction using staged subcutaneous tissue expansion followed by Deep Inferior Epigastric Perforator (DIEP) flaps. To show how this technique eliminates the patch-like appearance of the skin paddle.

\section{3}

\section{ALLOPLASTIC TEMPOROMANDIBULAR JOINT REPLACEMENTS}

\section{H Sinno, Y Tahiri, M Gilardino, D Bobyn}

PURPOSE: An overview of engineering concepts is presented to better understand the indications for surgical management, the types of available treatments and the requirements for reconstruction. Prosthetic implants are compared to autogenous reconstruction.

METHODS: A literature review of treatment of severe temporomandibular joint disorders (TMD) is done from the pubmed data base since 1950 to present. Biomedical engineering concepts are used to correlate with available reconstructive modalities.

RESULTS: 456 articles were found and cross referenced. The indications for TMJ replacement include: 1. ankylosis with severe anatomic abnormalites; 2. Failure of autogenous grafts; 3. Failure of ProplastTeflon or Vitek-Kent implants; and 4. Severe inflammatory joint disease that results in joint mutilation and functional disability. Advantages to using prosthetic implants over autogenous are discussed.

CONCLUSION: For the treatment of TMD, engineered TMJ alloplastic replacements have had considerable promise with additional room for improvement using new materials and recent design concepts.

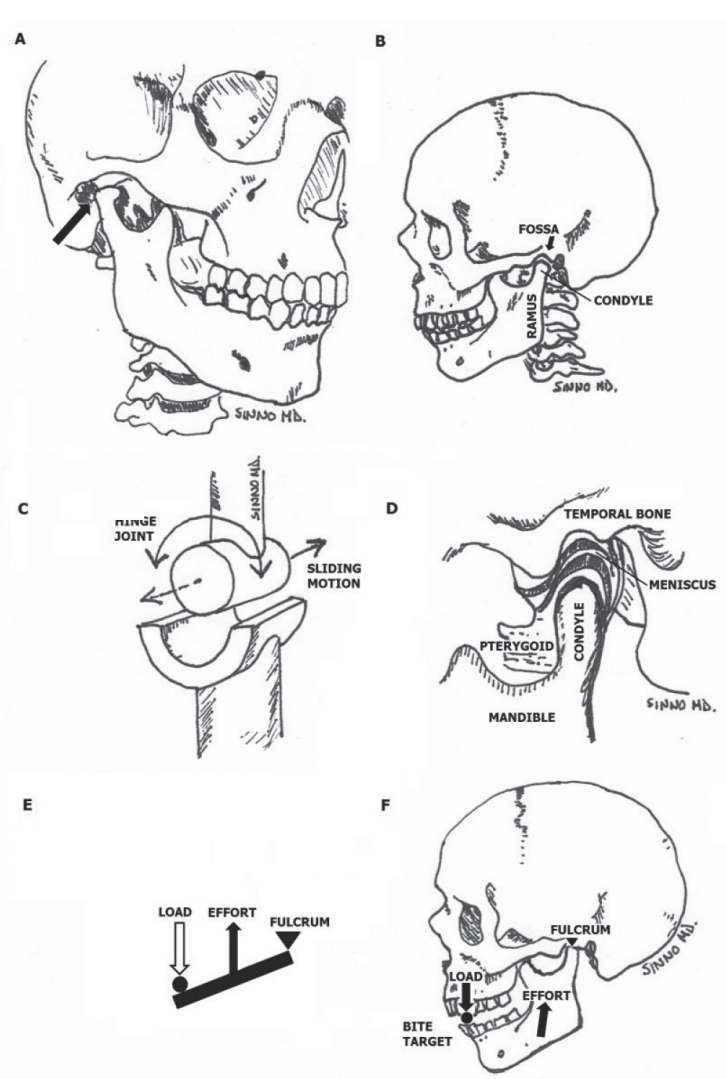

Figure 1) Functional Anatomy of the TMJ. A) Black arrow pointing to the anterolateral view of the TMJ as seen on a drawing of the human skull. B) Drawing of the left lateral TMJ on a human skull. C) A schematic of the TMJ as a hinge and sliding joint. D) Saggital section through the TMJ showing the meniscus and cartilage between the mandible and temporal bones of the TMJ. E) The jaw simplified as a third class lever: Lever: the mandible body; Fulcrum: the TMJ; Effort: force generated by the masseter muscle; and Load: the food bolus. F) Drawing of a lateral view of the human skull as a third class lever with a bite target as the load.

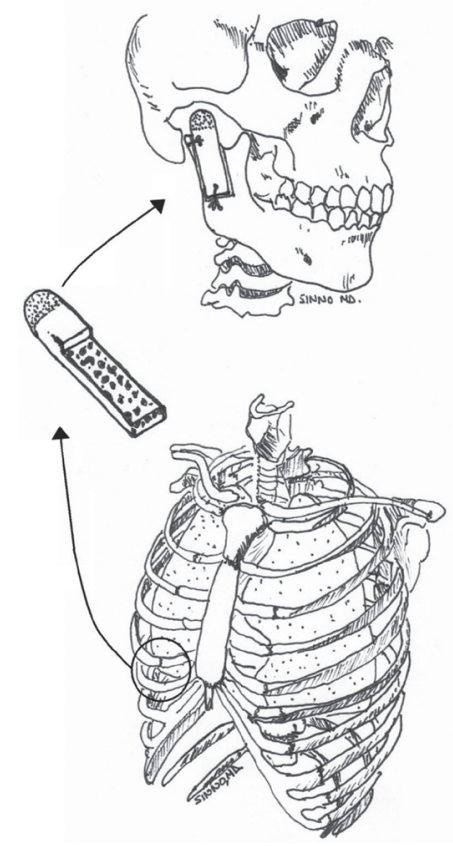

Figure 2) Autogenous TMJ Reconstruction. Drawing of the harvest of the costochondral rib graft for temporomandibular joint reconstruction. The decorticated rib graft is fit into the mandible as an inlay and fixed with $0.5 \mathrm{~mm}$ stainless steel wires. 


\section{TABLE 1}

\section{Indications for Alloplastic TMJ Reconstruction}

Ankylosis with severe anatomic abnormalities

Failure of autogenous grafts

Failure of Proplast-Teflon or Vitek-Kent or partial joint implants

Severe inflammatory joint disease that results in joint mutilation and functional disability

Learning Objectives:

- The participants should understand the engineering concepts of the TMJ and TMJ implants and their design.

- The participants should understand the indications of alloplastic TMJ arthroplasty.

\section{4}

INTERNATIONAL BURN RECONSTRUCTION: LESSONS LEARNED FROM AN INAUGURAL MISSION TO MUMBAI, INDIA AND FUTURE DIRECTIONS

RA Nelson, FS Kim, I Sinha, S Keswani, JF Watkins, AN Pandya

PURPOSE: To evaluate the outcomes of a foundational mission focused on burn scar reconstruction by reviewing patient assessment, operative planning, postoperative results, and follow-up care. Recommendations are made for future burn missions and feasibility is established.

METHOD: A retrospective case series analysis of patients admitted to the National Burn Center in Mumbai, India for Operation Restore/ Operation Smile during the period of January 8-18, 2010 was performed. Patient demographics, diagnoses and range of motions were evaluated. Postoperative complication rates were assessed and mission organization was reviewed.

RESULTS: Of 59 patients screened, 42 were candidates and 38 patients received surgery. The majority were female (88\%) with thermal burns resulting from kerosene stove blasts (70\%). Distribution of burn scar contractures included eyelid ectropion (5\%), oral commissure $(13 \%)$, neck (47\%), axilla (8\%), elbow (10\%), hand/wrist (34\%), lower extremity $(3 \%)$ and other $(24 \%)$. Treatment included the use of full-thickness skin grafts in $20(53 \%)$ patients, split-thickness grafts in $4(10 \%)$, z-plasties in $22(58 \%)$, Kirschner wires in $7(18 \%)$ and syndactyly release in $2(5 \%)$ patients. The most common postoperative complication was wound infection, occurring in $4(11 \%)$ patients. Range of motion outcomes for contracture releases were comparable with other studies.

CONCLUSIONS: International burn missions have a positive impact with acceptable patient outcomes in developing countries. Team members should be prepared to treat and rehabilitate burn contractures from all anatomic areas, using a variety of techniques. Local climate/humidity, patient hygiene and access to clean water may have an impact on postoperative infection rates and their complications, which should be considered when planning postoperative care. At all stages of patient assessment and treatment, cultural sensitivity is essential.

Learning Objectives:

- To identify a role for international missions in treating burn scar deformities.

- To recognize some of the differences in treating burn patients in developing countries.

\section{5}

\section{FURTHER VALIDATION OF AN INHALATION INJURY DIAGNOSIS AND GRADING COMPOSITE II SCORE}

\section{NG Jada, G Walia, R Cartotto}

INTRODUCTION: We have found that burn patients with ARDS have a compromised response to high frequency oscillatory ventilation (HFOV) if there was a bronchoscopy-confirmed inhalation injury (II). We have also described a composite II score which correlated with poor pulmonary outcomes. The purpose of this study was to determine if our composite II score also predicts the response to HFOV, as a further means of validating our II severity score.

METHODS: All adult burn patients with ARDS who received HFOV at our regional burn center between 11/10/99 and 30/4/09 were retrospectively scored for II severity using our previously reported composite scoring system which grades II as mild, moderate, or severe based on historical factors, physical findings, bronchoscopic severity, and $\mathrm{COHb}$ levels. RESULTS: There were 70 patients ( 36 mild, 22 moderate, 12 severe) with age $44 \pm 16$ yrs, \% TBSA $44 \pm 18$, and \% full thickness $34 \pm 21$, treated with HFOV. The mild group was older than the moderate group $(\mathrm{p}=0.01)$, but there were no other significant differences between groups in \% TBSA, \% full thickness burn, time to initiation of HFOV, or the pre-HFOV $\mathrm{PaO}_{2} / \mathrm{FiO}_{2}$ ratio (PFR) and Oxygenation Index (OI) on conventional mechanical ventilation $(\mathrm{CMV})$. OI declined in all 3 groups over time, with the mild group having a significantly lower OI only at hour $16(\mathrm{p}=0.02)$. However, within group comparisons revealed that neither the moderate nor severe group achieved significant reduction in the OI from that on CMV until after 48-96 hours of HFOV whereas the mild II group achieved stable and significant reduction in the OI from CMV within 16 hours of starting HFOV. The PFR of the mild group was significantly better than baseline CMV within 1 hour of HFOV, remaining significantly better to 96 hours whereas moderate and severe did not improve their PFR from CMV until after 24 and 48 hours of HFOV, respectively.

CONCLUSIONS: Patients with moderate and severe II had a blunted response to HFOV compared to those with mild II, similar to our prior observations which relied only on an arbitrary "present or absent" diagnosis of II based on bronchoscopy alone.

Learning Objectives:

- The findings further support the validity of our composite scoring system which diagnoses and grades the severity of an II. Also, the II severity score appears to predict which patients will respond poorly to HFOV.

\section{6}

EVALUATION OF KI-67 AS A NEW HISTOLOGICAL INDEX OF BURN DAMAGE IN A SWINE MODEL

H Farhangkhoee, KM Cross, V Koljonen, D Ghazarian, JS Fish

PURPOSE: Skin grafting is a surgical intervention used to facilitate wound healing and improve cosmesis in thermal injuries. The utilization of skin grafts is dependent on the degree of burn damage; however, histological diagnosis of burn depth lacks consensus. The purpose of this study was to determine whether $\mathrm{Ki}-67$, a cell proliferation marker, provides an index of integument viability following burn injury.

METHODS: Induction of thermal burn injuries (3, 12, 20, 30, 75, 90, 120 seconds) were made with a brass rod heated to $100^{\circ} \mathrm{C}$ on the dorsal trunk of the Swine. Controls were created with a brass rod heated to $37.5^{\circ} \mathrm{C}$. Four $6 \mathrm{~mm}$ biopsies were obtained from each site for histological analysis of $\mathrm{Ki}-67$.

RESULTS: Our results illustrate a characteristic Ki-67 nuclear staining in the basal layer of the epidermis and the hair follicle. With thermal injury of any duration, the nuclei of the cells changed morphology: condensing, fragmenting, and elongating. The uniqueness of the labeling index was to include only morphologically intact nuclei as having capacity to proliferation. Quantitative analysis showed a reduction in the mean number of $\mathrm{Ki}-67$ positive cells, suggesting a reduced regenerative capacity.

CONCLUSION: These results prove to be promising in developing a new histological marker for burn depth assessment.

Learning Objectives:

- This presentation will help the learner utilize Ki-67 as a histological marker of integument viability after burn injury in swine and to describe the significance of the hair follicle in wound regeneration. 
67

\section{A. Ross Tilley Lecture}

\section{SECURITIES AND SYNOSTOSIS: TWO YEARS OF CRANIOFACIAL SURGERY AND BIZ-SCHOOL \\ D Peters}

I completed two fellowships. In 2007-08 I was a clinical fellow at the Hospital for Sick Children in Toronto. This was conducted under the supervision of Drs. Christopher Forrest and John Phillips. I was involved in 86 major craniofacial osteotomy cases including craniosynostosis, cleft-orthognathic surgery and midfacial distractions. In 2008-09 I was a clinical fellow at Sunnybrook Hospital and Health Sciences Centre under the supervision of Drs. Oleh Antonyshyn and Jeffrey Fialkov. This fellowship consisted of craniofacial trauma, tumor reconstruction and secondary congenital cases. During that time I also completed an MBA at the Rotman School of Management at the University of Toronto. I learned the fundamental principles and practices of management allowing their application to medicine. I have since returned to practice in Canada at the University of Ottawa.

The 2007 Ross Tilley Fellowship was highly useful in providing an education in adult and pediatric craniofacial reconstruction as well as management.

Learning Objectives:

- To describe the way that the 2007 Tilley Scholarship was utilized.

- To present my experience in pediatric and adult craniomaxillofacial surgery.

- To present the potential for the interactions between business education and plastic surgery in the Canadian context.

\section{8 \\ NICORANDIL INDUCES LATE-PHASE ISCHEMIC PRECONDITIONING FOR PERIOPERATIVE PROTECTION OF SKELETAL MUSCLE FROM ISCHEMIA/REPERFUSION (I/R) INJURY}

NJ Cahoon, H Ashrafpour, N Huang, SOP Hofer, JE Lipa, CR Forrest, CY Pang

PURPOSE: During autogenous muscle transfer, unpredictable complications (e.g. vasospasm, thrombosis) can occur perioperatively, resulting in prolonged ischemic insult. Nicorandil (a nitrovasodilator and $\mathrm{K}_{\text {ATP }}$ channel opener) is known to induce $24 \mathrm{~h}$ uninterrupted infarct protection in myocardial models. Therefore, we investigated the efficacy and mechanism of Nicorandil in inducing perioperative protection of skeletal muscle from $\mathrm{I} / \mathrm{R}$ injury.

METHOD: Pigs ( $(18 \mathrm{~kg})$ were assigned to 1 control and 7 treatment groups ( $\mathrm{n}=4-5 \mathrm{pigs})$. Control pigs received i.v. saline injection. Treatment pigs received Nicorandil injection $(3 \mathrm{mg} / \mathrm{kg}$, i.v. $)$. Bilateral Latissimus Dorsi (LD) muscle flaps $(8 \times 13 \mathrm{~cm})$ were raised in control pigs immediately after saline injection and in treatment pigs at 8, 12, 24, 48, 72 and 96h after Nicorandil injection. Muscle flaps were subjected to $4 \mathrm{~h}$ ischemia/48h reperfusion. The muscle myeloperoxidase (MPO) activity, muscle ATP and mitochondrial free $\mathrm{Ca}^{2+}\left(\mathrm{mCa}^{2+}\right)$ contents were measured after $2 \mathrm{~h}$ reperfusion.

RESULTS: There was $40 \pm 2 \%$ infarction in muscle flaps subjected to $4 \mathrm{~h}$ ischemia/48h reperfusion (control). The muscle infarction was reduced to $22 \pm 2 \%, 25 \pm 2 \%$ and $28 \pm 2 \%(\mathrm{p}<0.05)$ when $4 \mathrm{~h}$ of ischemia was started at $24 \mathrm{~h}, 48 \mathrm{~h}$ and $72 \mathrm{~h}$ respectively, after Nicorandil injection. Compared with the control, this infarct protective effect of Nicorandil was associated with less neutrophil accumulation as indicated by lower muscle MPO activity $(0.41 \pm 0.01$ vs $0.96 \pm 0.07$ units/g); inhibition of $\mathrm{mCa}^{2+}$ overload ( $339 \pm 73$ vs $543 \pm 54 \mathrm{nmol} / \mathrm{mg}$ ); and preservation of ATP synthesis $(19.5 \pm 2.1 \mathrm{vs} 12.4 \pm 2.0 \mathrm{umol} / \mathrm{g}$ prot. $)$ ( $\mathrm{p}<0.05$ for all tests).

CONCLUSIONS: After $24 \mathrm{~h}$ of injection, Nicorandil induced $48 \mathrm{~h}$ uninterrupted protection of skeletal muscle from I/R injury. Nicorandil is a clinically available oral drug and could be a potential application for pharmacologically induced late-phase ischemic preconditioning against $\mathrm{I} / \mathrm{R}$ injury in elective free tissue transfer.
Learning Objective:

- To understand the concept of late-phase ischemic preconditioning and the role of pharmacological mimetics in this phenomenon.

\section{9}

\section{IN VITRO ASSESSMENT OF OSTEOBLAST BEHAVIOUR IN CRANIOSYNOSTOSIS}

TKS Cypel, I Leong, C Pang, P Dirks, C Forrest

PURPOSE: The importance of anti-osteogenic signalling molecules such as Noggin and BMP-3, in normal cranial suture fusion has recently been demonstrated in a murine model but the role of these molecules in pathophysiology of premature suture fusion (craniosynostosis) in humans remains unknown. The aim of this study was to investigate the regional variations in osteoblast function and the expression of osteogenic signalling molecules (noggin,BMP-3,Runx-2) in human infants with craniosynostosis.

METHODS: Bone and periosteal tissue from fused (FS), patent (PS) cranial sutures and adjacent bone (CB) were harvested from 31 infants (3-36 months old) undergoing cranial vault reshaping for craniosynostosis and used to develop primary osteoblast cell culture. Dural tissue was obtained from unrelated age-matched neurosurgical procedures $(n=10)$ in order to generate an osteoblast-dural co-culture. Osteoblast function and differentiation were assessed by proliferation (MTT), alkaline phosphatase, mineralization and osteocalcin production. Noggin, BMP3 and Runx-2 expression were assessed using immunohistochemistry and Western Blot.

RESULTS: Cell cultures demonstrated significant $(p<0.05)$ regional variations in osteoblast proliferation rates (FS:4.2>NB:2.5>PS:2.1 mean OD/1000cells), alkaline phosphatase production (FS:2.3>PS:1.9>NB:1.8 mean OD/1000cells) and in vitro bone nodule formation suggesting that FS osteoblasts demonstrated a higher propensity to differentiate and form bone. The expression of antiosteogenic molecules (noggin, BMP-3) was decreased in osteoblasts from FS regions. Expression of Runx-2 was increased in osteoblasts from FS in dural cell co-culture.

CONCLUSION: The results of this study suggest that important regional variations exist in osteoblast behaviour and differentiation in human infant with craniosynostosis. The creation of a pro-osteogenic environment through the decreased expression of anti-osteogenic signalling molecules (noggin, BMP3) and increased expression of osteogenic factors (FGF-2) may be responsible for premature suture fusion.

Learning Objectives:

- Assess differences in osteoblast behavior in vitro.

- Define the role of anti-osteogenic signalling in the pathophysiology of craniosynostosis.

\section{0}

PUBLICATION BIAS IN ABSTRACTS PRESENTED TO THE ANNUAL SCIENTIFIC MEETING OF AMERICAN SOCIETY OF PLASTIC SURGERY

A Izadpanah, H Sinno, A Izadpanah, M Gilardino

BACKGROUND: Publication bias is defined as favoring publication of research results based on their nature and direction (e.g. positive versus negative results). We sought to determine the presence of publication bias in the plastic surgery literature.

METHODS: Abstracts presented to the 2003 Annual Meeting of American Society of Plastic Surgeons (ASPS) independently were reviewed by two different reviewers. Abstracts were investigated for sample size, type of study, level of evidence, country of origin, presence of statistical results, and sponsorship. Publication within 5 years was ascertained by electronic search of Medline and Embase databases. In the absence of positive searching results, authors were contacted directly. Logistic regression analysis was used to identify predictors of publication.

RESULTS: There was no evidence of publication bias in the 2003 ASPS meeting. However, prospective, and blinding studies were more likely to be published subsequently. 


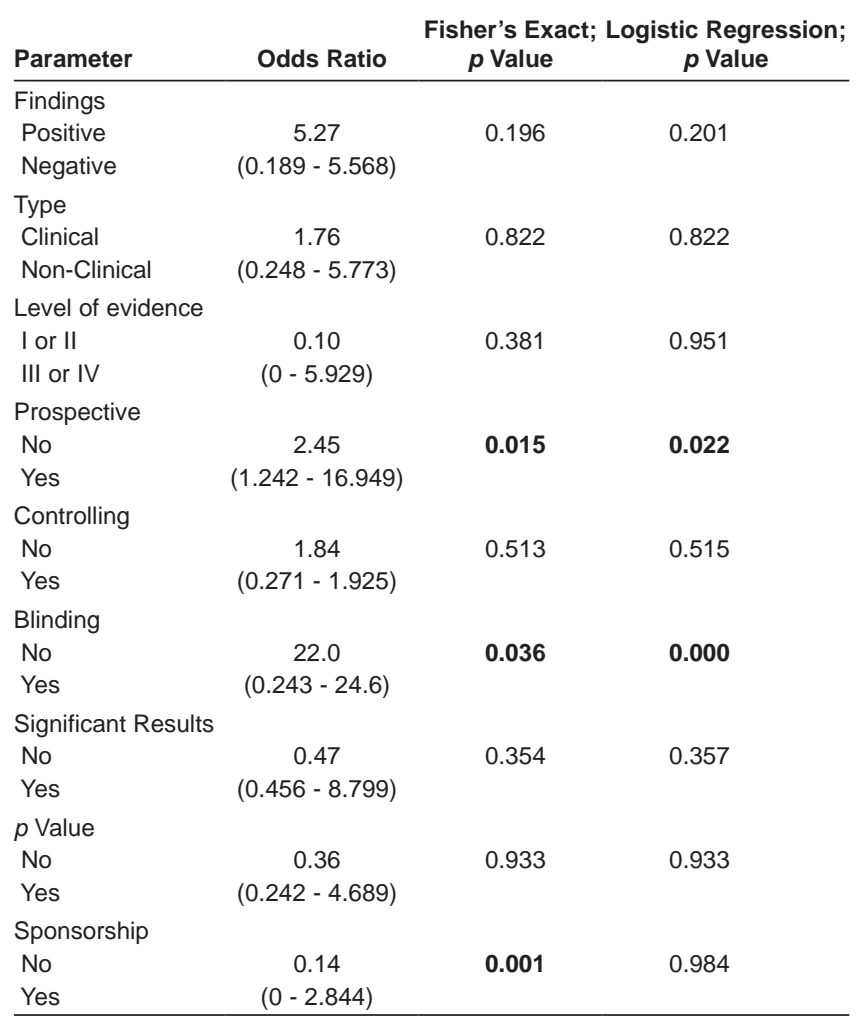

CONCLUSION: We found no evidence of publication bias in the review of manuscripts presented at the 2003 Annual Meeting of ASPS. Thus, researchers can be confident that the likelihood of acceptance will not be influenced by the direction of study findings.

Learning Objectives:

- Participants will be able to learn about publication bias and have a clearer idea of the potential determinants of manuscript publication.

\section{1}

BREAST RECONSTRUCTION AND RADIATION THERAPY: A DECISION ANALYSIS

\section{K Wong, T Zhong, B Graham}

PURPOSE: The ideal timing and type of breast reconstruction following mastectomy is unknown in the setting of potential post-mastectomy radiation (PMRT). Decision analysis is an effective approach to compare methods based on variable rates of complications and the probability of PMRT. Decision analysis also accounts for the value of various health states in the form of utilities. The purpose of this study is to compare methods of breast reconstruction with a decision analysis.

METHODS: A decision tree was created to compare: 1) immediate tissue expander/implant reconstruction (ITE), 2) immediate autologous tissue reconstruction (IATR), 3) delayed TE (DTE), and 4) delayed ATR (DATR). Variables for rates of complications and reconstructive failure were obtained from a systematic review of the literature. Utility values were obtained from the literature as well as expert opinion. Sensitivity analyses were performed to test the impact of varying the different variables on the outcome of the model.

RESULTS: In the base case analysis, IATR yielded the highest utility (0.7854), followed by DATR (0.7511), ITE (0.7414), and DTE (0.6707). However, the rates of complications varied widely in the 69 studies reviewed. In sensitivity analyses, the utility of ITE surpassed IATR when the rate of complications was $\leq 22 \%$ and the rate of failure was $\leq 5 \%$. ITE yielded higher utility than DATR when the probability of PMRT was $\leq 20 \%$.

CONCLUSIONS: ITE reconstruction yields the highest utility of the reconstructive methods when the rates of complications and failure are low. As the rates increase above $22 \%$ and $5 \%$ respectively, IATR gains superiority.
Learning Objectives:

At the end of this presentation, the learner will be able to:

- Understand the value of decision analysis in comparing outcomes.

- Use this decision analysis to determine the optimal method of breast reconstruction with PMRT.

\section{2}

DEMOGRAPHICS OF OROFACIAL CLEFTS (OC) IN CANADA FROM 2002 TO 2008

\section{S Pavri, CR Forrest}

PURPOSE: Orofacial clefts such as cleft lip (CL), cleft palate (CP), and cleft lip and palate (CLP) are the most frequent congenital anomalies of the head and neck. The purpose of this study was to determine the current regional and national demographics of OC in Canada.

METHODS: A request for data from the $\mathrm{DAD} / \mathrm{HMDB}$ databases from fiscal years 2002/03 to 2007/08 (excluding Quebec) was submitted to the Canadian Institute of Health Information. The data were analyzed using SAS 9.2. Variables evaluated included gender, cleft type, gestational age, birthweight, income quintile, and regional LHIN.

RESULTS: The incidence of OC ranges from 1.25 to 1.78 per 1000 live births (1:560 to $1: 800)$ across the country. Subdivided by cleft type, the proportions of patients with CL, CP, and CLP were, respectively, 20\%, $40 \%$, and $40 \%$. The female-to-male ratio of all OC births ranged from 0.67 to 0.92 , compared to a ratio of 0.95 for all Canadian newborns. Subdivided by cleft type, gender ratios averaged 0.56 for CL, 1.31 for CP, and 0.62 for CLP. While almost half of the total annual OC births occur in Southern Ontario, Saskatchewan and Manitoba were noted to have significantly higher OC birthrates $(\mathrm{p}>0.05)$ compared to Ontario and New Brunswick.

CONCLUSIONS: Canada has one of the highest OC birthrates in the world ( 1 in 685 live births). This study is the first to present an updated demographic of OC in Canadian newborns and may be useful in predicting the burden of health care expected in the management of these patients.

Learning Objectives:

- To understand the incidence and prevalence of OC in Canada.

- To understand epidemiologic and regional geographic influences in the incidence of $\mathrm{OC}$ in infants.

Each year an average of 400 of approximately 275,000 Canadian newborns are born with some type of orofacial cleft (OC) - including Cleft Lip (CL), Cleft Palate (CP), and Cleft Lip \& Palate (CLP). Based on national demographic data from CIHI's Discharge Abstract Database (DAD) - excluding data from Quebec, which was not available - the incidence of orofacial clefting (OC) across Canada from 2002 to 2008 ranged from 1.248 to 1.777 per 1000 births, and seems to fluctuate between its upper and lower bounds without any apparent trending pattern or known cause.

With only 11 dedicated cleft centers across Canada, the regional distribution of the total number of OC births is also important in determining burden of care. Almost half (between 159 and 211 - avg. 176 - from 2002 to 2008) of the total annual OC births in Canada occur in Ontario, with the majority concentrated in the southern part of the province. The number of $\mathrm{OC}$ births in the remaining provinces from 2002 to 2008 ranged as follows: Newfoundland 2-13 (avg. 7), P.E.I 0-4 (avg. 2), Nova Scotia 8-24 (avg. 13), Manitoba 29-36 (avg. 32), Saskatchewan 19-42 (avg. 28), Alberta 55-99 (avg. 70), British Columbia 56-87 (avg. 67), and Northwest Territories/Nunavut/Yukon $0-4$ (avg. 2). Most of the OC births followed the general distribution of population density, with concentrations around the provincial capitals and other large cities, most notably Calgary, Winnipeg, Vancouver, and Edmonton.

The gender ratio (female/male) of all OC births ranged from 0.67 to 0.92 from 2002 to 2008, compared to a ratio of 0.95 for all Canadian newborns. Subdivided by cleft type, gender ratios ranged from 0.46-0.75 (avg. 0.56) for CL, from 0.97-1.71 (avg. 1.31) for CP, and from 0.54-0.72 (avg. 0.62) for CLP, in accordance with CP being more prevalent in females and CL and CLP being more prevalent in males.

From 2002 to 2008, there was an average of 59 Cleft Lip births, 142 Cleft 
Palate births, and 144 Cleft Lip \& Palate births annually across Canada. There was no statistically significant difference in Gestational Age, Birthweight, or Economic Income Quintile between newborns with and without OCs, as well as between newborns with CL, CP, or CLP.

\section{3}

\section{CASE REPORT SERIES ON THE ANATOMY OF HAND CENTRAL SYNPOLYDACTYLY}

EP Egerszegi, $\underline{P}$ Grenier-Vallée

PURPOSE: Report our findings regarding the anatomy of hand central synpolydactyly including soft tissue and the bony involvement as well as arterial anatomy.

METHODS: Hand radiographs and angiograms of 5 children with central hand polydactyly, for a total of 8 hands, were reviewed. The cutaneous syndactyly present was classified according to the number of digits involved. The Buck-Gramco classification system for digital duplication was used to describe the central polydactyly present. We created a simplified classification system to organize the bony abnormalities found. The Coleman and Anson classification for the superficial arterial arch was used, and finally we developed a new classification system to describe the digital arterial patterns found.

RESULTS: All 8 hands presented a syndactyly including the supernumerary digit(s): 6 involved 3 digits, 1 involved 4 digits, 1 involved 5 digits. In every hand, bony malformations were present and classified according to the modifications we propose to the Buck-Gramco system. Regarding the Coleman and Anson classification for the superficial arterial arch, there was one hand with an incomplete arch and the 7 others had a complete arch. We identified several arterial patterns in the fingers and all included missing arteries.

CONCLUSIONS: Some of the digital arterial anomalies found have a direct repercussion on the surgical treatment plan. We therefore recommend systematic hand angiography in the preoperative evaluation of hand central synpolydactyly.

Learning Objectives:

At the end of this presentation the learner will be able to:

- Recognize and classify the bony anomalies present in hand central synpolydactyly using the simplified method presented.

- Describe and classify the digital arterial anomalies common in hand central synpolydactyly.

- Recognize the importance of angiography in the preoperative evaluation of hand central synpolydactyly.

Canadian Expert Series

\section{SEX REASSIGNMENT - FEMALE TO MALE MASTECTOMIES AND CHEST CONTOURING \\ C Bowman}

\section{5}

\section{SUP-ER SPLINTING: DOES THE USE OF A SPLINT THAT ENCOURAGES SUPINATION AND EXTERNAL ROTATION IN CHILDREN WITH OBSTETRIC BRACHIAL PLEXUS PALSY HAVE BENEFIT?}

\section{A Benson, D Bellows, K Durlacher, C Verchere}

PURPOSE: Birth related brachial plexus injury (BRBPI) affects about $1 / 1000$ live-born children with injury to one or more branches of the brachial plexus. Most children demonstrate a good functional outcome by one year. Even in these patients, many will retain lifelong limitation in passive and active range of shoulder external rotation and forearm supination, often with secondary skeletal changes. We have piloted a novel infant splinting technique which aims to increase these ranges of movement in the short and longer term by positioning the forearm and shoulder into supination and external rotation (SUP-ER splint) during the early months of recovery. The concept is similar to infant splinting for clasped thumb and developmental dysplasia of the hip.
METHOD: Nine consecutive patients with incomplete recovery of their brachial plexus injury at 2 or more months were enrolled in our study. Presplinting and serial measurements were taken during the therapy period using the Hospital for Sick Children Active Movement Scale. Patients undergoing brachial plexus exploration were excluded. The SUP-ER splint was applied prior to 6 months of age and ideally less than 3 months. Most children terminated splint use prior to 9 months of age.

RESULTS: The splint was generally well tolerated but compliance was variable in this pilot study. In all patients, range of movement scores improved over the study period. There were no complications observed during the splinting period. Patients with greater compliance achieved earlier improved movement scores in external rotation and supination. Long term follow-up is ongoing.

CONCLUSION: The SUP-ER splint may be beneficial to prevent shoulder and forearm limitations in children with BRBPI. Interest is welcomed at a national level for a multi-centre trial to evaluate this promising technology.

Learning Objectives:

- To describe a novel splinting technique for birth related brachial plexus injury.

\section{6}

SKULL BASE SCOLIOSIS IN UNICORONAL SYNOSTOSIS IS Wheeler, T Samson, S Blazer, C Forrest

PURPOSE: Anterior plagiocephaly due to unicoronal synostosis (UCS) is typified by ipsilateral forehead flattening, periorbital asymmetries, nasal deviation and temporal bulge. The skull base assumes an asymmetric, twisted appearance due to the abnormally fused suture. The purpose of the study was to characterize the skull base changes in UCS following cranial remodeling. We performed a Computed Tomography (CT)-based morphometric analysis to characterize the skull base deformity before and after surgery.

METHODS: CT scans were obtained in patients with plagiocephaly (UCS, positional, lambdoid synostosis) between the years of 1999-2008. Using a workstation the following key measures were obtained, i) axial skull base angle measured from Opisthion-anterior Sella-Foramen Caecum, ii) nasal angle measured from a line between the ZF sutures and a line from nasion through the Anterior Nasal Spine.

RESULTS: The average axial angle measured was 16 degrees preoperatively in UCS $(n=48)$ where normal should be 0 degrees $+/-2$ degrees. A follow-up scan performed in patients with UCS post-surgery at 12 months or greater showed an average axial angle of 12 degrees $(p>0.05)$. Preoperatively, the nasal angle was 97 degrees and post-operatively at 1 year or greater the nasal angle was 94.5 degrees. Age at surgery was not a variable. Ten patients with marked positional plagiocephaly had an axial angle averaging 2 degrees. Five patients with isolated lambdoid synostosis had an average axial angle of 175 degrees.

CONCLUSIONS: The skull base deformity in UCS is more marked than in other plagiocephalic conditions. Cranial base remodeling postsurgery was noted.

Learning Objectives:

- To become familiar with the skull base changes seen in an infant with unicoronal synostosis.

- To understand CT-based morphometric techniques.

\section{7}

\section{FUNCTIONAL OUTCOME AFTER THE HOFFER PROCEDURE}

\section{A Murabit, K O'Grady, M Morhart, JL Olson}

PURPOSE: Children with obstetrical brachial plexus injury often develop an internal rotation and adduction contracture about the shoulder as a secondary deformity. This, combined with the incomplete recovery of deltoid and rotator cuff muscles leads to an inability to externally rotate and abduct the shoulder. The Hoffer procedure is evaluated for its potential benefit in improving shoulder abduction and external rotation and its impact on activities of daily living.

METHODS: This study is a retrospective review of patients treated in 
our Brachial Plexus Injury Clinic who underwent the Hoffer tendon transfer procedure. Epidemiological data were collected and analyzed regarding the patient and their injury. Age at time of surgery, surgical complications and other surgical procedures are catalogued. Preoperative and post-operative active movement and active ROM are measured and recorded using the Mallet scale and AMS.

RESULTS: This study included 20 patients: 18 underwent the Hoffer procedure; 2 had a L'Episcopo procedure. 11 of the patients were male, 9 female. Age ranged from 3-15 years old. All patients had full or almost full passive ROM pre-operatively. Average follow-up period was 23 months. Average improvement in aggregate Mallet scores was 4.74 points. Active range of motion in shoulder abduction improved on average 2.11 points on the AMS; and external rotation improved 4.00 points on the AMS. Active internal rotation changed on average -0.63 points on the AMS, however all patients maintained full ROM passively, thus no functional loss was experienced. Better pre-operative shoulder flexion and shoulder internal rotation yielded better postoperative results. Younger patients ( $\leq 6$ years) yielded better results.

CONCLUSIONS: The Hoffer procedure can provide improved external rotation and abduction while preserving functional internal rotation range in the child with obstetrical brachial plexus palsy and secondary shoulder deformity.

Learning Objectives:

- Understand the secondary deformity of the shoulder in patients with obstetrical brachial palsy.

- Understand the indications, surgical technique and peri-operative care for the Hoffer procedure.

- Demonstrate the results of the Hoffer procedure in addressing the secondary shoulder deformity caused by obstetrical brachial plexus injury using the Mallet scale and the Active Movement Scale (AMS).

\section{8}

THE EPIDEMIOLOGICAL PROFILE OF CHILDREN PRESENTING WITH VASCULAR BIRTHMARKS IN THE CALGARY HEALTH REGION

\section{R Flannigan, F Fraulin, V Sharma, R Harrop}

PURPOSE: To review the characteristics of patients seen in the Vascular Birthmark clinic (VBMC) at the Alberta Children's Hospital $(\mathrm{ACH})$.

METHOD: A retrospective data analysis of all patients presenting to the VBMC between 1998 and 2009 was performed. Data were obtained from the clinic's database that records demographic, referring, diagnostic and treatment information.

RESULTS: The VBMC database included 1029 patients: 621(60.3\%) hemangiomas, 311(30.2\%) vascular malformations, and 97(9.4\%) other diagnoses. The majority of patients were referred from Southern Alberta 788/932 (84.5\%), with 7\% from central Alberta, 1.6\% from Northern Alberta and 6.9\% from outside Alberta (BC, SK, MB, Other).

The 621 patients with hemangiomas had 880 lesions. Most patients $(68.5 \%)$ were female. The most common anatomical location was head and neck $478 / 880$ (54.3\%) followed by trunk (21.8\%), extremities $(21.1 \%)$ and perineal region $(2.4 \%)$. Overall 91/621 (14.7\%) patients underwent diagnostic imaging investigation. $147(23.7 \%)$ patients required treatment either medications, dressings, surgical excision or pulsed dye laser.

The 311 patients with vascular malformations had an almost equal sex distribution: $154 / 311$ (49.5\%) female: (50.5\%) male. Venous malformations accounted for $45 \%$ of patients, isolated capillary malformations $31.5 \%$, lymphatic malformations $12.5 \%$ and arteriovenous malformations $2.9 \%$. Mixed capillary-venous-lymphatic malformations - Klippel Trenaunay Syndrome - occurred in 8\% of patients. Overall $116(37.3 \%)$ patients underwent diagnostic imaging investigation and 132 (41.8\%) required treatment - either pulsed dye laser, sclerotherapy, surgical excision or dressings.

CONCLUSIONS: The VBMC of ACH serves a large geographical area. The majority of patients seen have hemangiomas and almost $1 / 4$ needed treatment. Vascular malformations make up about $1 / 3$ of the clinic patients and are more likely than hemangiomas to require some type of treatment.
Learning Objectives:

- To understand the relative frequency of hemangiomas and vascular malformations.

- To identify groups of these patients requiring investigation and treatment.

\section{9}

MORBIDITY OF INVASIVE SQUAMOUS CELL CARCINOMA OF THE SCALP

\section{K Elzinga, CD McKenzie, JG McKinnon, J Arlette}

PURPOSE: Squamous cell carcinoma (SCC) of the scalp can exhibit aggressive behavior, metastasize, and cause death. SCCs of the scalp can be treated with Mohs micrographic surgery (MMS) to confirm histologically clear local margins and maximally preserve tissue. However, local control does not predict patient outcome. This study aims to establish which factors may predict patient outcome.

METHOD: The charts of 182 patients who have been treated with MMS for SCC of the scalp were reviewed. The analysis included the location, pathological subtype, presence of perineural or galeal involvement, defect size, repair type, and patient co-morbidities, including immunosuppression. These factors were correlated with patient morbidity and mortality due to local disease, in-situ metastases, and distant spread.

RESULTS: 93 of the SCCs were located on the parietal skull, 60 were frontal, 18 temporal, and 11 occipital. Based on the pathology reports, of the invasive SCCs, 45 were well differentiated, 21 moderately differentiated, and 18 poorly differentiated. There were also 17 atypical SCCs (mucoid, acantholytic, spindle cell, basosquamous, endophytic, and clear-cell squamoproliferative SCCs). To repair the defects, 129 were closed primarily, while 53 were repaired with advancement and rotation flaps, as well as split and full thickness skin grafts. 45 of the SCCs demonstrated galeal invasion, while perineural invasion was present in 19 SCCs.

CONCLUSIONS: This review reports on the largest series of patients with SCC of the scalp treated by MMS reported in the literature. It represents an advancement in the therapy of SCC of the scalp for those at high risk of morbidity and mortality.

Learning Objectives:

- Participants will be able to describe risk factors that increase morbidity for patients with SCC of the scalp.

- Participants will be able to determine which patients with SCC of the scalp would benefit from further treatment, such as radiotherapy or chemotherapy, after MMS.

\section{0}

\section{EOSINOPHILIC CELLULITIS (WELLS SYNDROME) DIAGNOSIS AND TREATMENT: CASE REPORT AND LITERATURE REVIEW}

IP Lacroix, H Sinno, R Borsuk, K Watters, M Gilardino

INTRODUCTION: Eosinophilic cellulitis (Wells syndrome) is a recurrent inflammatory dermatitis that resembles cellulitis and as a result may prolong adequate diagnosis and treatment.

METHODS: We present a case report and a review of the literature of all the idiopathic cases of eosinophilic cellulitis since 1950 retrieved from the Pubmed and Medline database.

RESULTS: Patients present with or without systemic symptoms (fever, arthralgia, malaise) and the lesions may or may not be accompanied by blisters, bullae, papules or nodules. The rate of success of the different treatments include: steroids $(91.7 \%)$, dapsone (60\%), topical corticosteroids $(50 \%)$ anti-histamine $(25 \%)$ or continuous low-dose systemic corticosteroid may be used in case of persistent recurrences.

CONCLUSIONS: Antibiotic resistance cellulitis must trigger a suspicion of Wells syndrome. Where the most successful treatment includes a short course of systemic corticosteroid.

Learning Objectives:

- At the end of this lecture, the learner will be able to assess the differential diagnosis of Wells syndrome. The participant will also learn how to administer the appropriate treatment regarding the patient's history and complaints. 
Posters

\section{P01}

THE USE OF FILLERS FOR THE PRIMARY CORRECTION OF NASAL DEVIATION IN AN 18 YEAR OLD MIDDLE-EASTERN MALE

\section{」Piggott, A Yazdani}

PURPOSE: Rhinoplasty in the Middle-Eastern male patient poses challenges due to unique anatomy, and patient wishes to retain an unoperated, Middle-Eastern appearance. The objective of this case report is to demonstrate a case of filler use for primary correction of a nasal deviation.

METHODS: Restylane was used for primary correction of right-sided nasal deviation in an 18 year old Lebanese male with a previous nasal bone fracture. Injections of Restylane were placed above the depressed left nasal bone, and at the radix.

RESULTS: The nasal deviation was corrected, Middle-Eastern and masculine appearance of the nose were maintained, with patient satisfaction, low cost, and no complications.

Learning Objectives:

- The objectives of this report are to highlight the unique characteristics of a Middle-Eastern male nose and to offer a minimally invasive option for the correction of a deviated nose.

\section{P02}

\section{RISK FACTORS INFLUENCING TRANSFUSION RATES IN DIEP FLAP BREAST RECONSTRUCTION}

\section{SE Appleton, A Ngan, B Kent, SF Morris}

BACKGROUND: As the popularity of DIEP flap breast reconstructions grows, there remains little information on the transfusion rate of this more technically challenging procedure. The purpose of this study was to examine the peri-operative blood losses and patient characteristics of women undergoing DIEP flap breast reconstructions in an attempt to identify independent risk factors that predict increased blood loss and transfusion requirements.

METHODS: A retrospective chart review demonstrated 144 patients who underwent DIEP flap breast reconstructions performed at QEII Health Sciences centre between January 2002 and July 2009. Intraoperative blood losses, hemoglobin and hematocrit drop, length of hospital stay, incidence of peri-operative blood transfusions and complications were reviewed. Both univariate and multivariate analysis were conducted to assess the effect of older age, obesity, bilateral, reconstruction, immediate reconstruction and concurrent treatment with Tamoxifen on the incidence of peri-operative blood transfusion and complications.

RESULTS: $18.8 \%$ of patients required peri-operative blood transfusions. These patients were older, more obese, underwent more immediate and bilateral reconstructions, and had longer mean operative times and hospital stays. Patients undergoing bilateral reconstructions were at increased risk and greater odds of receiving a blood transfusion (relative risk 3.2, odds ratio 4.4). Blood transfusion recipients had an increased risk and greater odds of experiencing any post-operative complication (relative risk 2.3, odds ratio 3.4).

CONCLUSION: Bilateral breast reconstruction was the only factor to significantly increase the risk of peri-operative blood transfusion. Also, receiving a blood transfusion was associated with an increase in the risk of experiencing any post-operative complication.

Learning Objectives:

- Understand the increased risk of blood transfusion in DIEP flap breast reconstructions.

- Understand which risk factors increase the risk of peri-operative blood transfusion.

- Understand link between peri-operative blood transfusion and the incidence of any complication.

\section{P03}

\section{PERCUTANEOUS ELECTRICAL STIMULATION ACCELERATES AXON REGENERATION FOLLOWING CARPAL TUNNEL RELEASE SURGERY IN HUMANS}

HA Power, C Pilipchuk, DC Edwards, J Olson, M Morhart, T Gordon, KM Chan

PURPOSE: Peripheral nerve regeneration is slow and functional recovery is often poor following injury. Electrical stimulation (ES) of injured nerves accelerates axonal regeneration in laboratory animals. Although we recently demonstrated similar effects in humans through submaximal ES, in this study we further investigated the efficacy of ES in humans following carpal tunnel release surgery (CTRS) using a novel stimulation technique.

METHODS: Twenty-two patients with severe median nerve compression in the carpal tunnel were recruited for this randomized control trial. Those in the control group received open CTRS only, while the 11 patients in the treatment group also received $20 \mathrm{~Hz}$ ES through a percutaneous monopolar needle electrode adjacent to the median nerve at the elbow for $1 \mathrm{~h}$ at maximal tolerable intensity. Patients were followed at regular intervals for 1 year. Axonal regeneration was quantified using motor unit number estimation (MUNE).

RESULTS: The stimulation group showed significant axonal regeneration at 3 months post-operatively with the MUNE increasing from $113 \pm 75$ at baseline to $159 \pm 84$, whereas the control group did not significantly improve even after 1 year $(\mathrm{p}=0.31)$. The extent of reinnervation was no different between stimulation at the elbow and the wrist.

CONCLUSIONS: Post-surgical ES accelerates axonal regeneration and muscle reinnervation following CTRS. Stimulation of the median nerve at the elbow produced similar benefits as stimulating at the wrist. This proof-of-principle study confirms that ES has a significant physiologic effect on nerve regeneration in humans. It may be a clinically useful adjunct to surgical repair for other peripheral nerve injuries where functional recovery with conventional treatment is poor.

Learning Objectives

- At the end of this session, the learner will understand the challenges in treating peripheral nerve injury and how axonal regeneration may be enhanced by a new adjunct to surgery.

\section{P04}

LOSS OF ARTICULAR CARTILAGE - CLOSED PIP JOINT INJURIES OF THE HAND

\section{B Byers, JB Clapson}

1. The primary purpose of this study was to perform a retrospective review of $\mathrm{x}$-rays of closed PIP joint injuries obtained from a community plastic surgery practice, and document the extent of cartilaginous injury. The secondary purpose of the study was to document all other significant radiographic findings in attempt to appreciate the breadth and extent of hand injuries in general.

2. The amount of joint space loss was ascertained by manually measuring the joint space of the injured joint, then comparing these values to those of the adjacent non-injured joints. This was achieved using an advanced electronic radiological program.

3. We found that $26 \%$ of injured PIP joints showed a joint space narrowing of greater than $25 \%$ in comparison to the contiguous DIP joint, and $22 \%$ of injured PIP joints were narrowed by a margin greater than $33 \%$. We also found that $68 \%$ of our subjects had an "other" finding not associated with the PIP joint of interest, suggestive of old injuries such as bone avulsions, fractures, joint deformities, osteoarthritis, subluxations, soft tissue growths, and osteomas. There appears to be a correlation with the age of the patient as $92 \%$ of subjects over the age of 50 had other findings.

4. The finding of joint space loss supports the belief that closed PIP joint injuries are often associated with poor outcomes, ultimately leading to post-traumatic arthritis. The extent of other radiological findings supports the notion that hand injuries are very common, the history of which is often preserved on subsequent $\mathrm{x}$-rays. 
Learning Objectives:

- Appreciate the damage inflicted upon the articular cartilage following a closed PIP joint injury as evidenced by joint space loss.

- Appreciate the breadth of other radiological findings from a sample of subjects with PIP joint injuries.

\section{P05}

\section{INVASIVE ASPERGILLOSIS OF A DIGIT}

\section{T Sattler, D Tobbia}

PURPOSE: For the first time a case of invasive Aspergillosis of a digit is reported.

METHOD: Case report.

RESULTS: A 47 year old male immunosuppressed patient suffering from anaplastic Astrocytoma tripped in the garden and injured the distal phalanx of his left middle finger. Initially he presented to the family doctor where he was treated conservatively and received oral antibiotics. Six weeks later the patient presented to our department with a necrotic non-healing lesion to the middle finger. Radiologic examinations in two planes didn't show any signs of osteomyelitis. A partial amputation of the left middle finger was performed just distal to the insertion of the FDS tendon. A specimen was sent for pathology and microbiology. A few days later the patient was discharged under appropriate antibiotic coverage.

Three weeks after our surgical intervention the patient showed signs of excessive intracranial pressure due to his fast growing anaplastic astrocytoma and died.

Postmortem the final histology report confirmed the diagnosis of invasive Aspergillosis of the distal phalanx of the left middle finger.

CONCLUSIONS: There are many publications on Aspergillus infections in immunosuppressed patients. This is the first reported case of an invasive Aspergillus infection of the bones of a digit.

Learning Objectives:

- The Plastic Surgeon should be aware of uncommon infections especially in immunosuppressed patients.

- In non-healing infections despite appropriate antibiotic coverage tissue samples should be sent for pathology.

\section{P06 \\ PROMINENT EAR CORRECTION: OPERATIVE AND POSTOPERATIVE TECHNIQUES FOR IMPROVING OUTCOMES AND PATIENT SATISFACTION}

\section{K Shokrollahi}

We describe a number of innovations in Otoplasty. One relies on skin glue instead of head bandages as post-operative splints. In effect, the ears are glued to the side of the head after copious application along the suture line and posterior ear. We offer this to all patients as an alternative to bandages, and most prefer it - especially females, because they can wear their hair long which hides all stigmata of surgery and they can also shower on the first postoperative day as well as resuming all other activities of daily living and work. Furthermore, there are no problems with patient re-attendences for bandages that have fallen off, nor the discomfort of reduced hearing and sometime nausea. The glue splint lasts for between 2 and 3 weeks and there have been no complications in 25 sequential cases. The author has yet to meet a surgeon who has tried glue and not incorporated it into their practice routinely.

Of perhaps greater interest is the experience of 40 patients operated upon with a new technique of Otoplasty which relies on the adjunctive use of an anteriorly based post-auricular fascial flap which is sutured to the mastoid process. This allows precise manipulation of ear shape and contour as well as projection. Furthermore, it acts as an internal splint for the ear, complementing the glue-ear splint above, as well as providing cover for suture knots in otoplasty techniques that rely on buried knots which can often extrude. This poster provides a detailed anatomical description and cadaveric study of the postauricular fascia, demonstrates the operative technique involving the postauricular fascial flap, and demonstrates the use of glue as a postoperative splint. The two techniques are complimentary.
In Summary:

PURPOSE: To disseminate experience of a number of innovations in otoplasty to the benefit of patients.

METHODS: A postauricular fascial flap otoplasty technique combined with postoperative splinting of ears with glue in favour of head bandages.

RESULTS: Complete satisfaction from patients and surgeons with glue as splints without complication. 1 unilateral recurrence and no other complications in 40 otoplasty techniques with good results illustrated by operative examples.

Learning Objectives:

- For those who may wish to use them, these techniques offer a number of advantages that can replace or supplement aspects of individual surgeons' otoplasty practice.

\section{P07}

\section{THE USE OF BIOSYNTHETIC SKIN SUBSTITUTE (BIOBRANE) IN THE TREATMENT OF EXFOLIATIVE SKIN DISORDERS}

\section{Tobbia, T Sattler, R Cartotto}

INTRODUCTION: Toxic epidermal necrolysis (TEN) is a rare but potentially life threatening dermatologic disease that is part of a spectrum of drug related exfoliative disorders including Steven-Johnson syndrome (SJS) and SJS/TEN overlap. The cutaneous manifestations are characterized by a variable degree of epidermal detachment at the dermoepidermal junction, resulting in microscopic changes comparable with those that occur in superficial dermal burns, justifying the need for patient management in a specialized burn unit. The main objective of this study was to determine the time required for the wounds to heal and the infection rates when using Biobrane ${ }^{\circledR}$ (smith \& nephew, London, UK) for skin coverage.

METHODS: A retrospective chart review of exfoliative disorders from January 1999 to December 2007 was performed. The parameters included in the study were patient demographics, time from disease onset to burn unit admission, total surface area of detached skin and/or mucosal involvement, the area and anatomy covered with Biobrane, healing time, complications, mortality and outcome.

RESULTS: A total of thirty-two patients were identified with either TEN or SJS/TEN overlap, nineteen were female and thirteen were male. The patients studied had a mean age of $55.7 \pm 21.1$ years (range, 18 to 86 years). Time from initial onset of symptoms to burn unit admission was $7.2 \pm$ 3.36 days (range, 3 to 18 days), body surface area of epidermal detachment was $41 \% \pm 23 \%$ (range, $10 \%$ to $100 \%$ ). Twenty-eight patients $(87.5 \%$ ) required wound coverage with Biobrane, the mean surface area covered was $39.2 \% \pm 20.9 \%$ (range, $10 \%$ to $90 \%$ ), time from admission to complete reepithelialization in the surviving patients was $10 \pm 3.9$ days (range 5 to 20 days). Only four patients (14.3\%) developed a wound infection, and only two of those required complete removal of Biobrane.

CONCLUSION: In this susceptible group of patients, skin coverage with a synthetic skin substitute such as Biobrane early in the course of treatment provides barrier protection, helping to reduce wound infection rates and promote rapid healing.

\section{P08}

COMPARING TRANSAXILLARY ENDOSCOPIC ASSISTED TECHNIQUE WITH THE INFRAMAMMARY APPROACH FOR BREAST AUGMENTATION: 16 YEAR EXPERIENCE

H Sinno, A Izadpanah, A Izadpanah, VW Papanastasiou

BACKGROUND: The transaxillary endoscopic assisted breast augmentation technique has been shown to be a safe and reliable method. The literature fails to justify its increased cost and necessity. In the sixteen year experience of one surgeon, two methods for breast augmentation are reviewed.

METHODS: From 1993 to 2009, 625 patients underwent breast augmentation (mean age $33.4 \pm 9.54$ years). $260(24.6 \%)$ patients (mean age $33.8 \pm 6.45$ years) opted for the inframammary incision approach, $22(10.6 \%)$ of which also received a mastopexy for ptosis correction. 
$365(58.4 \%)$ patients (mean age $33.2 \pm 7.36$ years) chose the transaxillary endoscopic approach. The mean follow up time was $12.1 \pm$ 16.9 months.

RESULTS: Although not statistically significant, the cost associated with the transaxillary endoscopic approach was higher than the cost associated with the inframammary augmentation $(p=0.129)$. The average implant size for the inframammary approach was $283 \pm 64.68 \mathrm{cc}$ as compared to the transaxillary endoscopic technique $330 \pm 66.49 \mathrm{cc}$. Complication rate for the inframammary technique in comparison to the transaxillary technique included abnormal scar $(n=25,10.68 \%$ vs. $\mathrm{n}=8,2.9 \%$; likelihood ratio $=26.5, \mathrm{P}<0.001)$ capsular contracture $(\mathrm{n}=$ $22,8.46 \%$ vs. $\mathrm{n}=19,6.78 \%$; likelihood ratio $=7.1, \mathrm{P}=0.037$ ), infection $(\mathrm{n}=5,1.9 \%$ vs. $\mathrm{n}=7,2.5 \%$; likelihood ratio $=1.3, \mathrm{P}>0.05)$, hematoma ( $\mathrm{n}=5,1.9 \%$ vs. $\mathrm{n}=10,3.6 \%$; likelihood ratio $=2.5, \mathrm{P}>0.05)$, seroma $(\mathrm{n}=0)$ nipple-areolar complex sensational change $(\mathrm{n}=11,4.2 \%$ vs. $\mathrm{n}=7$, $2.5 \%$; likelihood ratio $=2.7, \mathrm{P}=0.326)$, minimal malposition $(\mathrm{n}=36$, $13.8 \%$ vs. $\mathrm{n}=30,10.7 \%$; likelihood ratio $=3.36, \mathrm{P}>0.05)$ and major or minor revisions ( $n=19,7.3 \%$ vs. $n=25,8.9 \%$; likelihood ratio of 2.5 and $\mathrm{P}>0.05$ ).

CONCLUSION: Both the inframammary and transaxillary incisions are relatively safe procedures with high patient satisfaction. The transaxillary incision can be an option for patients who do not desire breast scarring and do not mind the extra cost. In the face of ptosis however, the transaxillary approach is not indicated. Transaxillary approach is associated with a statistically significant lower rate of abnormal scaring and capsular contracture.

Learning Objectives:

- At the end of this lecture/workshop, the learner will be able to compare the indications for inframammary verses the endoscopic transaxillary approach for breast augmentation.

- At the end of this lecture/workshop, the learner will be able to compare the complications found with the inframammary verses the endoscopic transaxillary approach for breast augmentation.

\section{P09}

\section{TGF-BETA CORECEPTOR CD109 REGULATES} EXTRACELLULAR MATRIX PRODUCTION IN A SKIN FLAP-INDUCED HYPOXIA WOUND MODEL

\section{S Winocour, J Vorstenbosch, A Trzeciak, L Lessard, A Philip}

PURPOSE: Our group has identified a novel TGF- $\beta$ antagonist CD109 which inhibits extracellular matrix (ECM) protein production in vitro. Since some fibrotic skin disorders such as scleroderma are associated with hypoxia, we examined whether CD109 is able to regulate ECM deposition in transgenic mice overexpressing CD109 in the skin, under low oxygen tension.

METHOD: Transgenic mice and wild-type littermates were used to generate a hypoxic wound model by creating dorsal bipedicle skin flaps with centrally located excisional wounds. Excisional wounds were made in each experimental group: (i) without skin flaps $(n=6)$, (ii) with skin flaps but no underlying silicone sheet $(n=6)$, and (iii) with skin flaps having an underlying silicone sheet $(n=6)$. Mice were sacrificed at 7 or 14 days after surgery, and tissues were harvested for analysis. The degree of hypoxia was evaluated by HIF-1 $\alpha$ levels and histological changes were analyzed by $\mathrm{H} \& \mathrm{E}$ staining. Finally, alterations in ECM protein levels and CD109 expression were determined by Western blot.

RESULTS: Both transgenic and wild-type mice on days 7 and 14 postwounding showed increased levels of HIF-1 $\alpha$ in hypoxic excisional wounds, validating this animal model in mice. Transgenic mice on day 7 post-wounding demonstrated decreased collagen I and fibronectin deposition, and reduced dermal thickness in hypoxic wounds as compared to their wild-type counterparts, and to non-hypoxic internal control wounds. Finally, CD109 expression levels inversely correlated with the degree of hypoxia found in wounds in both transgenic and wild-type mice.

CONCLUSIONS: Hypoxic wounds in transgenic mice display decreased ECM content, as well as decreased dermal thickness, suggesting that CD109 inhibits ECM production under hypoxia. As a result, manipulating this molecule may have potential therapeutic value for the treatment of fibrotic skin disorders which are associated with poor oxygen delivery.

Learning Objectives:

- To understand the effect of CD109 overexpression on hypoxic wound healing.

\section{P10 \\ DISTAL RADIUS RECONSTRUCTION WITH VASCULARIZED FREE PROXIMAL FIBULA FLAP: A CASE REPORT AND LITERATURE REVIEW}

Lutfy, O Fouda Neel, H Sinno, M Luc

PURPOSE: Excision of the distal radius for the treatment of osteosarcoma in children has two main reconstructive challenges: 1) maintenance of joint function and 2) potential for forearm growth. Autogenous vascularized proximal fibula free flap with physis for reconstruction of the pediatric distal radius is an important surgical technique which meets the aforementioned criteria. We describe the steps of our reconstruction and summarize the expected long-term outcomes based on the literature.

METHOD: A 9 year-old girl presented with a distal radius osteosarcoma extending up to the wrist joint. The plan was for an oncological resection of the distal radius and reconstruction utilizing a vascularized free proximal fibula flap. A two teams approach, orthopedic and plastic surgery, performed the procedure. Challenges observed included the tedious dissection of the proximal fibula underneath the common peroneal nerve and in establishing a retrograde venous flow after flap insetting.

A literature review using Ovid was performed and all articles with similar presentation are discussed including their methods and results.

CONCLUSIONS: When indicated, this procedure is safe with satisfactory range of motion of wrist and forearm growth observed at follow-ups in both the case presented and in the literature.

Learning Objectives:

At the end of this presentation, participants will become familiar with the:

- Requirement of maintaining joint function and growth potential in pediatric oncologic wrist reconstruction.

- Unique blood supply of the proximal fibula and its growth plate from the anterior tibial artery.

- Challenges associated with reverse free flap venous anastomosis when venous flow is retrograde.

- Difficulties of harvesting the proximal fibula while preserving all the branches of the common peroneal nerves.

- Long-term outcomes of the procedure based on the literature.

\section{P11}

CHRONIC AND IRREDUCIBLE DORSAL PROXIMAL INTERPHALANGEAL JOINT (PIPJ) LUXATION OF THE FINGER: A REPORT OF 3 CASES

M Murphy, P Benoit, $\mathrm{H}$ St. Amand

PURPOSE: Luxation injuries of the PIPJ are common and often successfully reduced with immediate treatment. However, a minority of patients present with late stage sequelae. As such, closed reductions become impossible due to periarticular soft tissue contracture and necessitate an open procedure. To this end, we report our experience involving 3 cases and a brief review of treatment options.

METHOD: A retrospective study with chart review was performed for 3 males aged $42-47$ years old (average 44.2 years) presenting with chronic dorsal PIPJ luxation involving either the index, middle or small finger. Average time from injury to surgery was 15.7 weeks (range: $7-32$ weeks). After review of treatment options and post-operative goal assessment, all three elected to have replacement arthroplasty with a silastic implant. Range of motion (ROM) and strength will be assessed at one year post surgery (average, $48 \mathrm{wks}$; range, 34-57 wks).

RESULTS: At initial presentation, all patients had rigid PIP joints $\left(0^{\circ} \mathrm{ROM}\right)$. Six weeks post surgery, the average ROM improved to $32.5^{\circ}$ (range $20-45^{\circ}$ ). Grip strength and 3-point pinch measured for one patient, as a percentage of the non-injured hand, were $32 \%$ and $48 \%$, respectively. 
CONCLUSIONS: Chronic PIPJ dorsal luxation without concomitant fracture is rarely reported in the literature. Alternate surgical methods include volar plate arthroplasty and dynamic distraction via external fixation followed by open reduction with extension block splinting, resulting in an average final $\mathrm{ROM}$ of $60.5^{\circ}$ and $75^{\circ}$ at 59 and 152 weeks, respectively. Our preliminary report describes increased ROM and function 6 weeks post surgery. Cases will be followed out to one year and compared with the aforementioned surgical management techniques.

Learning Objectives:

- Participants will be able to identify the presentation of the chronic/irreducible PIPJ and the sequelae involved, describe the current treatment strategies and recognize the goals for functional outcome.

\section{P12 \\ HIGH-VOLTAGE ELECTRICAL INJURY TO BILATERAL UPPER EXTREMITIES: CASE REPORT AND REVIEW OF THE LITERATURE}

J Lee, L Kanevsky, A Perkins, H Sinno, Y Tahiri, M Luc

PURPOSE: We encountered a 47 year old Hydro Quebec worker male, who while working on a pole fell down and grabbed electrical cords to avoid failing. He suffered a 14000 volt injury to both hands. Our purpose is to evaluate the serious consequences of high-voltage injury to extremities and a review of the literature on current management methods.

METHOD: Medline search of high-voltage electrical injury and current management trends.

RESULTS: We describe a case of a 47 year-old electric company linesman who suffered a high voltage electrical injury (HVEI) of 14,000 volts to bilateral hands and wrists managed at our trauma centre. His management included multiple operative procedures, including escharotomies, fasciotomies, serial debridements, and bilateral pedicle groin flaps, and amputation of his left hand. In addition, we set out to review the literature on high-voltage electrical injuries.

CONCLUSIONS: High-voltage electrical injuries can have serious comorbidities, significant hospital stays and a lengthy rehabilitation period. We review the current treatment managements for this serious type of injury.

Learning Objectives:

- Participants will be able to provide a comprehensive review of the literature on current management of high-voltage electrical injuries.

- Participants will be able to explain our rationale and our methods for treatment of high-voltage electrical injuries.

- Participants will understand the difficulty and inevitable complications of high-voltage electrical injuries.

\section{P13}

\section{ATYPICAL FIBROXANTHOMA; NOW WHAT DO I DO?}

\section{Dempsey, J Davidson}

PURPOSE: Atypical fibroxanthoma (AFX) is an uncommon skin tumour occurring typically in the head and neck region of elderly Caucasian males. While the disease is generally considered to have a benign course, it can occasionally demonstrate aggressive biologic activity including local recurrence as well as distant metastasis. Some have characterized AFX as a variant of malignant fibrous histiocytoma rendering uncertainty as to what constitutes adequate treatment of this entity. At this time there are no known prognostic factors to guide surgeons in the management of patients who present with this neoplasm.

METHODS: We have reviewed our experience with AFX in Kingston, Ontario with regard to patient demographics, anatomic location of the lesion, depth of invasion at initial biopsy, and time course of recurrence.

RESULTS: $\mathrm{N}=71 ; \mathrm{M} / \mathrm{F}$ ratio $=4: 1$ : local recurrence $=10(14 \%)$; distant metastasis $=3(4 \%)$.

CONCLUSION: From this analysis we have proposed treatment guidelines to assist surgeons in managing patients with this confusing and sometimes challenging diagnosis.
Learning Objectives:

- Know more about an uncommon cutaneous neoplasm.

- Know the prognostic factors that predispose to treatment failures.

- Understand basis for proposed treatment guidelines.

\section{Residents Poster Corner}

\section{RP01}

\section{PYODERMA GANGRENOSUM AFTER BREAST AUGMENTATION: CASE REPORT AND REVIEW OF THE LITERATURE}

\section{LS Paek, H Sinno, A Perkins, H Al-Ajmi, K Schwarz}

PURPOSE: This is a case of a 49-year old female who after secondary breast augmentation mastopexy developed pyoderma gangrenosum. Pyoderma gangrenosum (PG) is a rare idiopathic skin disorder that is a difficult diagnosis. The current appropriate diagnostic procedures involve tissue biopsy and histologic analysis. Our purpose is to present a case report of PG after breast augmentation mastopexy and review the literature.

METHODS: This is a case report and review of the literature of a 49-year old female after secondary breast augmentation mastopexy developed PG.

RESULTS: This is a case report and review of the literature of a 49-year old female after secondary breast augmentation mastopexy developed pyoderma gangrenosum (PG). We reviewed the literature on the presentation, diagnosis and management of PG.

CONCLUSIONS: PG is an elusive diagnosis, it is an idiopathic neutrophilic dermatosis. It has a unique presentation and tissue specimen is required for diagnosis. Current treatment management involves immunosuppression by steroids.

Learning Objectives:

- To present our unique case of PG after secondary breast augmentation mastopexy procedure.

- To review the literature associated with PG and their presentation and diagnosis.

- To discuss the current non-surgical management of PG.

\section{RP02}

\section{A SURVEY OF CURRENT PRACTICE PATTERNS IN DIAGNOSIS AND INTERVENTIONS OF INHALATION INJURIES IN CANADIAN BURN CENTRES}

\section{ŁK Yeung, A Papp}

BACKGROUND: Mortality and morbidity in burn victims are greatly increased with concominant smoke inhalation injuries. However, there has been a lack of academic progress in the clinical management in this field. Specifically, there has not been any studies that compiled current practices in North America. The aim of this study is to summarize current Canadian practice patterns in diagnosis and interventions of inhalation injuries.

METHODS: A questionnaire was sent to the medical directors of all 16 burn centres across Canada regarding the diagnosis and interventions of inhalational injuries.

RESULTS: $50 \%$ of respondents required bronchoscopy for confirmation of diagnosis of inhalational injury. All of the bronchoscopies were performed by staff; $87.5 \%$ by the Intensive Care Unit and $37.5 \%$ by Plastic Surgery. Most common indications for intubation were physical exam $(87.5 \%)$ and clinical history $(75 \%)$. The physical exam features thought to be most consistent with inhalation injuries were dyspnea and hoarseness $(87.5 \%)$. Common treatment included intubation $(87.5 \%)$, routine ventilatory support $(87.5 \%)$ and chest physiotherapy $(75 \%)$. None of the centres used nebulized heparin. Only a minority of centres who responded have a fluid resuscitation protocol (37.5\%). A quarter of Canadian Burn Centres (25\%) routinely use prophylactic antibiotics for all inhalation injuries.

CONCLUSION: In Canada, physical exam is the most common tool used in the diagnosis of inhalation injuries. Although bronchoscopy is the gold standard, it is only utilized $50 \%$ of the time for diagnosis. There is need for further research to generate a consensus for diagnosis. 
Treatment for inhalation injuries are fairly consistent across the nation; however, there is still a lack of protocol in fluid resuscitation.

Learning Objectives:

- Participants will be able to identify physical exam features consistent with inhalational injuries.

- Participants will be able to summarize current practice patterns in diagnosis of inhalational injuries.

- Participants will be able to summarize current interventions in inhalational injuries.

\section{RP03 \\ RETROSPECTIVE ANALYSIS OF FLUID RESUSCITATION IN MODERATE TO SEVERE ADULT BURN PATIENTS}

\section{K Sass, P McBeth, A Kirkpatrick, D Nickerson}

PURPOSE: Given the devastating nature of burn injuries and their primary morbidity, it is important to minimize essentially correctable and preventable secondary complications, such as abdominal compartment syndrome. We wish to identify local current practices involving adult burn patients requiring ICU admission to answer two primary questions: 1) Are adult burn patients receiving higher than predicted volumes of fluid during initial resuscitation?; 2) Are there higher rates of edema-related complications? Scientific recognition of existing inadequacies in clinical management will hopefully lead to the institution of regulatory guidelines and patient tracking software which can be used to guide all clinicians and health care personnel involved in the care of burn patients towards better patient management, thus minimizing morbidity and mortality.

METHODS: Our study is a retrospective analysis of current practices at the Foothills Medical Centre in Calgary involving adult burn patients requiring ICU admission over the past 10 years. We are accessing both prehospital and ICU charts to extract epidemicological, physiologic and pharmacologic data over the initial 48 hour resuscitation period. Secondary outcomes of assessment will include associated complications (pulmonary edema, extremity compartment syndrome, wound sepsis, pneumonia, abdominal compartment syndrome). A spreadsheet database will be created from which we will perform multivariate statistical analysis and calculations.

RESULTS: In progress. Based on the literature and general clinical observations by local burn and trauma surgeons, we hypothesize that: adult burn patients are receiving fluid volumes in excess of those predicted; that there is inappropriate fluid titration in response to urine output; and, because of these factors, that there is a tendency toward higher rates of edema-related complications, i.e., abdominal compartment syndrome.

CONCLUSIONS: In progress.

Learning Objectives:

- Participants will be able to identify potential existing inadequacies in current burn care resuscitation of adult patients.

- Participants will be able to initiate change in the management of these patients.

\section{RP05 \\ ARTERIAL VERSUS VENOUS CONDUITS IN MICROVASCULAR SURGERY: AN EXPERIMENTAL STUDY} LZhu, LF Zhu, P Kwan, M Morhart

INTRODUCTION: To bridge segmental arterial defects, the current gold standard involves using venous interposition grafts. However, there are multiple theoretical reasons why arterial conduits would be superior in connecting vessel gaps. Arterial grafts are easier to handle, produce more anti-thrombic substances, and undergo less endothelial cell detachment. Some surgeons believe that these factors warrant the use of arterial interposition grafts in complex microvascular cases, such as thumb replantation.

OBJECTIVE: The objective of this study is to compare arterial to venous interposition grafts in terms of flap survival in an experimental model of the rat.

METHODS: Thirty male Sprague-Dawley rats were assigned to a control and experimental group. Axial fasciocutaneous flaps were raised based on the femoral and superficial epigastric vessels. A $4 \mathrm{~mm}$ segment of the arterial pedicle was removed. In the control group, a vein graft was interposed between the two ends. In the experimental group, an arterial graft was used. Both the vein and artery had identical vessel diameters. The outcome measurement was flap viability, as determined on post-operative day 7 .

RESULTS: The rate of flap necrosis was 11 of 15 in the control group and 4 of 15 in the experimental group $(p=0.027)$. The relative risk reduction was $63.6 \%$.

CONCLUSION: Within this experimental model, the risk of thrombosis was significantly reduced when using an arterial conduit. Results of this study support the theoretical advantages offered by arterial grafts when compared to vein grafts.

Learning Objectives:

- Participants will understand the theoretical advantages of arterial grafts in comparison to vein grafts.

- Participants will understand the purpose of using an thrombogenic modification for this study.

\section{RP06}

\section{A COMPARISON OF 2\% LIDOCAINE, 0.5\% BUPIVACAINE, AND A COMBINATION OF 2\% LIDOCAINE/0.5\% BUPIVACAINE IN DIGITAL BLOCKS}

\section{A Tung, E Brown}

PURPOSE: Local anaesthesia is critical for various surgical procedures. This study examines the pharmacokinetics and pain on injection of lidocaine, bupivacaine, and their combination.

METHODS: A randomized controlled trial was carried out on 14 volunteers. Each received four digital blocks, with a random order of $1 \mathrm{cc}$ solutions consisting of $2 \%$ lidocaine, $0.5 \%$ bupivacaine, a $1: 12 \%$ lidocaine $/ 0.5 \%$ bupivacaine combination, and normal saline. Pain on injection was recorded ( 1 to 5 ), and numbness then self-assessed via pinprick ( 1 to 4 ) over time. Onset was defined as the average time required for complete numbness (4), whereas duration was the average time required to return to "normal" sensation (1). One volunteer's data was discarded due to a recording error.

RESULTS: As expected, normal saline did not result in significant numbness. Onset was 3.11 minutes for 2\% lidocaine, 18.46 minutes for $0.5 \%$ bupivacaine, and 5.58 minutes for the combination, and was significantly different between $2 \%$ lidocaine and $0.5 \%$ bupivacaine $(p=0.037)$ only. Duration was 183.23 minutes for $2 \%$ lidocaine, 853.85 minutes for $0.5 \%$ bupivacaine, and 706.15 minutes for the combination, and was significantly different between all solutions. Pain on injection was 2.7 for $2 \%$ lidocaine, 3.1 for $0.5 \%$ bupivacaine, 2.7 for the combination, and 2.6 for normal saline, and was not significantly different.

CONCLUSION: Our data confirms that $2 \%$ lidocaine has a quicker onset and shorter duration than $0.5 \%$ bupivacaine. Their combination results in a solution that is slower to take effect than $2 \%$ lidocaine (although not statistically significantly), and does not convey the full benefit of $0.5 \%$ bupivacaine's duration. The ideal solution, with both short onset and long duration, is seemingly not created by mixing lidocaine and bupivacaine, likely due to their competitive pharmacokinetic nature. Therefore, it would be more useful to select the suitable agent for the appropriate situation.

Learning Objectives:

- To understand the pharmacokinetics of local anaesthetics in order to choose suitable agents for specific situations.

\section{RP07}

SMILE VECTOR PLANNING - A TRANSLATIONAL STUDY AS Nimigan, DB Matic

PURPOSE: Much clinical and research effort has targeted surgical restoration of the smile in facial paralysis. Many well described flap and sling options exist and new options continue to arise. There remains, however, uncertainty in many aspects of smile reconstruction. Deciding on the vector of pull for a natural appearing active smile is a challenging step which factors heavily in post operative symmetry. 
METHODS: Three approaches to smile vector analysis were undertaken. A cadaveric study of four fresh heads allowed vector measurements of zygomaticus major (ZM) and minor muscles. Thirty live faces were then measured to assess the vectors of the mid-lip and commissure during active smile. Finally, a subset of this live group was then measured again after undergoing local anaesthetic block of depressor anguli oris $(\mathrm{DAO})$ and orbicularis oris $(\mathrm{OOr})$ muscles to investigate the effect on verticality of the smile vector.

RESULTS: Angles of interest were measured relative to the Frankfurt plane. The cadaveric group had an average $\mathrm{ZM}$ angle of $51+/-5^{\circ}$. Clinical measurements in the thirty adults showed mid-lip and commissure vectors of $39+/-9^{\circ}$ and $37+/-7^{\circ}$ respectively. No significant differences were noted between sexes or left and right sides. After local anesthetic block of DAO and OOr muscles, smile vectors became more vertical approaching those of the cadaveric group.

CONCLUSIONS: This translational research study takes fresh cadaveric and live anatomic findings into the realm of clinical application in smile reconstruction. In the non-paralyzed face, the smile vector is a balance between the more vertical pull of the ZM muscle with the more horizontal influence of lip sphincter and depressor muscles. Using the $\mathrm{ZM}$ as a guide, the ideal placement of the dynamic vector of pull should therefore be more horizontal to mimic the natural smile.

Learning Objectives:

- After hearing this talk, listeners will better understand the anatomy of the perioral and nasolabial muscles.

- After hearing this talk, listeners will recognize the influence of different facial muscles on the position of the smile.

\section{RP08 \\ SURGICAL SITE INFECTIONS IN CRANIOFACIAL FRACTURE PATIENTS: A RETROSPECTIVE REVIEW}

\section{H Baltzer, M Oyewumi, M Elahi, J Mahoney, M Musgrave}

PURPOSE: Variable follow-up of craniofacial fracture patients presents challenges to identification and treatment surgical site infections (SSIs). The aim of this study is to evaluate clinical criteria for diagnosis of infections following craniofacial fracture surgery and to examine patient detection of SSI.

METHODS: All craniofacial fracture patient files from 2004-2009 were reviewed retrospectively for rates of diagnosed SSI (gold standard), objective clinical findings consistent with CDC criteria of SSI, and patient perceptions of infection.

RESULTS: The rates of SSI based on documented clinical diagnosis and documented CDC criteria were $6.7 \%$ and $20.1 \%$, respectively. SSI diagnosis was associated with $>1$ concurrent, objective CDC criteria, erythema, pain, and purulent drainage $(\mathrm{p}<0.05)$. Presentation of CDC criteria was significantly later in patients diagnosed with infection $(\mathrm{p}<0.05)$, most of which were deep SSIs requiring secondary procedures (70\%). Eighteen percent of patients thought they had SSIs. In these patients, delayed presentation, subjective purulent drainage, subjective edema, and greater than one subjective CDC criteria were associated with SSI diagnosis $(p<0.05)$. The distribution of SSI diagnoses among CFF groups was uneven, with mandible SSIs over-represented (78\% of SSIs, $\mathrm{p}<0.05)$. Potential, contributing factors for the mandible group included smoking, mental health issues, substance abuse, shorter interval between injury and OR, and pre- and post-operative antibiotic use $(\mathrm{p}<0.05)$.

CONCLUSIONS: CDC diagnosis and patient diagnosis of SSI overestimate true rates; however, the presence of specific objective and subjective findings, particularly with delayed presentation, are important for influencing SSI diagnosis. Higher rates of SSI in mandible patients may be related to socioeconomic and clinical factors. These findings highlight the need to educate patients about symptoms of infection and the need for consistent follow-up.

Learning Objectives:

- To recognize the CDC criteria for SSI.

- To review the presentation of SSI following mandible ORIF.

- To contribute to patient education regarding SSI.

\section{RP09}

\section{FACTORS AFFECTING PRIORITIZATION FOR} CONSULTATION REGARDING CARPAL TUNNEL SYNDROME B Chung, SF Morris

PURPOSE: To determine existing factors by which plastic surgeons in Canada prioritize referrals for consultation regarding carpal tunnel syndrome (CTS).

METHOD: All members of the Canadian Society of Plastic Surgery with e-mail addresses were sent an invitation to participate in an online survey on factors that affect the methods by which they prioritize referrals regarding CTS. All members who did not respond to the survey within 2 weeks were sent a reminder notice to encourage their participation.

RESULTS: A total of 150 members responded to the survey $(40 \%$ response rate); 118 (79\%) stated that they performed CTS surgery. The majority of respondents who perform CTS surgery prioritize their consultation list chronologically (77\%). Factors that would alter the chronological order in which patients are prioritized included: subsequent contact by the referring physician (24\%); a personal relationship with the patient $(16 \%)$; and specific information in the referral letter (15\%), which was most commonly comment about severity of symptoms or EMG findings. Of the respondents who do not see referrals for CTS in chronological order, "severity of disease" was a prevalent theme amongst described factors on which prioritization was based. Forty-two percent of plastic surgeons stated that there was no conscious decision on how they came to choose the method of prioritization they use for referrals regarding CTS. CONCLUSIONS: There are a variety of factors that influence the priority order in which referrals for CTS are seen amongst CSPS members. However, the rationale for the methods by which CSPS members prioritize these referrals is poorly understood. Further study on developing evidence-based prioritization methods may be useful in assisting surgeons and their patients in outcome-based decisions.

Learning Objectives:

- Participants will be able to identify factors on which prioritization of CTS referrals are based.

\section{RP10}

\section{THE PREVALENCE OF MUSCULOSKELETAL DISORDERS IN CANADIAN PLASTIC SURGEONS}

\section{A Pirani, N Sharma, E Brown}

PURPOSE: The purpose of this study is to determine the prevalence of neck, back, and leg pain in Canadian plastic surgeons. The study also seeks to describe protective/predisposing factors associated with such musculoskeletal disorders in this population.

METHODS: A national survey of 308 plastic surgeons in Canada was conducted using an online survey tool. The anonymous survey included questions regarding general demographics, scope of practice, health status, and questions based on a validated chronic pain questionnaire.

RESULTS: A total of 122 plastic surgeons completed the survey $(39.6 \%$ response rate). $64.8 \%$ of respondents indicated at least one episode of musculoskeletal pain over the last 6 months. The majority of this pain was attributable to lower back pain $(42.3 \%)$ and neck pain (49.6\%). Of those with musculoskeletal pain, $67.7 \%$ identified their occupation as a contributing factor to their musculoskeletal pain. $31.7 \%$ of respondents had imaging confirmed pathology. $67.5 \%$ of respondents had sought medical treatment for their musculoskeletal pain. $6.5 \%$ of respondents had required surgical treatment. Only $8.1 \%$ of respondents reported missing work in the previous six months due to their musculoskeletal pain. $24.4 \%$ of respondents felt that their occupational longevity would be reduced due to their musculoskeletal pain. $53.8 \%$ of respondents believe that they would benefit from increased access to ergonomically designed operating room equipment.

CONCLUSIONS: Musculoskeletal pain is a significant issue amongst Canadian plastic surgeons, particularly lower back pain and neck pain.

Learning Objectives:

At the end of this presentation, the audience will be able to:

- Characterize their own musculoskeletal pain using a validated pain assessment tool.

- Identify potential sources of musculoskeletal pain in their work environment. 


\section{RP11}

MAJOR UPPER LIMB NERVE INJURY IN CENTRAL AND NORTHERN ALBERTA BETWEEN 2006 AND 2008

\section{R Guilfoyle, H Power, M Chan, J Olson, M Morhart}

PURPOSE: This study was designed to elucidate the incidence, mechanism, and treatment of patients who sustained brachial plexus injury or its major branches in Edmonton Central and Northern Alberta over a 3-year period.

METHOD: A retrospective chart review was performed on all adult $(\geq 18)$ patients seen at the Edmonton Brachial Plexus Clinic between 2006 and 2008. Exclusion criteria were peripheral nerve injuries in the upper limb involving only minor nerve branches or digital nerves.

RESULTS: A total of 60 patients were seen, yielding an average annual incidence of 2 per 100,000 . The majority were younger adults $(40 \%$ were $18-35$ y.o.) with a male preponderance of $68 \%$. The most frequent mechanism of injury was motor vehicle collisions (36\%), followed by falls $(30 \%)$. Over half $(54 \%)$ of patients were treated conservatively, while the remainder underwent surgical reconstruction. The mean wait time from date of injury to surgical treatment was 323 days.

CONCLUSIONS: With almost exclusive capture of this patient population in Central and Northern Alberta, this is one of the few studies in which the incidence of brachial plexus injury could be established. The patient characteristics and mechanisms of injury found were in keeping with those reported at other centers. The average wait time of $10-11$ months from date of injury to surgery is longer than our goal of 6-9 months. Further work is being done to delineate barriers to treatment and to expedite care.

Learning Objectives:

- This study will allow the reader to become familiar with the epidemiological characteristics of brachial plexus injury in Central and Northern Edmonton Alberta as they pertain to incidence, mechanisms of injury, and length of time to surgical treatment.

\section{RP12}

\section{TECHNIQUES FOR DETERMINING PLASTIC SURGERY} WORKFORCE REQUIREMENTS IN CANADA

\section{K Cheung, A Thoma}

PURPOSE: To identify an appropriate method for determining the workforce requirements for plastic surgeons in Canada.

METHODS: We conducted a literature review to identify the techniques used to calculate workforce requirements for any surgical field. Studies from the past 30 years indexed in Medline, EMBASE, or Cochrane databases were extracted and reviewed. Relevant medical subject headings $(\mathrm{MeSH})$ were identified and references were reviewed. RESULTS: 25 primary studies were identified; only 2 were specific to plastic surgery. Previous techniques may be classified as need-based, demand-based, or benchmarking assessments. Demand-based assessments use current and historical utilization rates as an indicator of physician requirements. Needs-based assessments rely on expert opinion to estimate future physician requirements to manage projected disease burden. Benchmarking compares current regional workforces to regions or health systems that have been identified as efficient.

While studies employing these techniques have accounted for the myriad of factors influencing physician supply and demand (e.g. the projected patient population, disease burden, sex and generational differences in physician work patterns, and operating room availability), they do not account for the factors unique to plastic surgery. A workforce equation for plastic surgery must consider cosmetic and reconstructive cases, variations in practice patterns, public demand for elective procedures, and potential overlap with otolaryngology and occuloplastics. Accurate plastic surgery workforce calculations should also have an objective measure for determining physician need. A measure of patient preference or wait times may provide this objectivity.

CONCLUSION: Determination of plastic surgery workforce requirements in Canada necessitates consideration of a number of factors that are unique to our specialty. Previous techniques will require modification to account for these factors.
Learning Objectives:

- Understand the factors that influence physician supply and demand including those specific to plastic surgery.

- Recognize the strengths and limitations of previous techniques for determining physician workforce requirements.

\section{RP13}

\section{THE EXTENT OF PRACTICE VARIATION FOR THE REDUCTION MAMMOPLASTY AMONG PLASTIC SURGEONS IN HAMILTON AND COST ANALYSIS OF POST OPERATIVE CARE}

\section{CP White, J Bain, A Thoma}

PURPOSE: The purpose of this study is to identify the variation in practice for the reduction mammoplasty among plastic surgeons in Hamilton, Ontario and to calculate and compare the cost for post operative care.

METHODS: A survey of all plastic surgeons in the city of Hamilton, Ontario, Canada who perform the reduction mammoplasty was conducted. Data were collected on preoperative selection criteria, intraoperative surgical techniques, methods and materials and post operative specific patient management. Details on patient follow up, appointments, use of post operative antibiotics and pain medications were also collected. The data collected was used to estimate total cost to the health care system with regards to post operative care. Responses were analyzed and common elements were identified. These were used to implement a common health care pathway for all patients in Hamilton undergoing a reduction mammoplasty.

RESULTS: The results were obtained from nine plastic surgeons in Hamilton who perform reduction mammoplasty. There was marked variance in all levels of the survey. These included using pre-printed orders (5/9), use of post operative heparin (5/9) and number of times patient seen in follow up. Certain commonalities were noted such as the use of surgical site drains (8/9), not operating on active smokers (7/9), post admission oral antibiotics (7/9) and home nursing support (7/9). For other elements like post operative pain management there was no consistency seen. Post operative care patterns were calculated and compared for all nine surgeons showing the difference in cost of health care dollars for the management techniques.

CONCLUSIONS: This study showed several variations in practice with regards to pre, intra and post operative techniques with regards to the reduction mammoplasty preformed by surgeons in Hamilton.

Learning Objectives:

- Participants will be able to define variations in reduction mammoplasty post operative care.

- Participants will be able to identify cost associated factors for reduction mammoplasty post operative care.

\section{RP14}

RESTORATION OF EXTERNAL ROTATION IN MASSIVE CUFF TEAR PATIENTS WITH GRACILIS FREE-FUNCTIONING MUSCLE TRANSFER: AN ANATOMIC FEASIBILITY STUDY

\section{DM Tremblay, P Tétreault, JC Lin}

PURPOSE: Free-functioning muscle transfers (FFMT) have been used for the restoration of function in such varied conditions as facial paralysis, brachial plexus paralysis and incontinence. Rotator cuff tear is a common injury that leads to shoulder joint arthropathy and is associated with pain, decreased range of motion and loss of shoulder function. Surgical treatment options exist, but none have proven completely satisfactory to relieve the symptoms of cuff tear arthropathy. We hypothesize that the supraspinatus muscle could be replaced by a gracilis FFMT, which may help to relieve the symptoms of rotator cuff tear.

METHODS: We dissected six shoulders and six gracilis muscles on three cadavers to determine the feasibility of our hypothesis. We first began by dissecting a free gracilis muscle flap to objectify muscle bulk, length as well as pedicle length and size. We then determined a surgical approach to the shoulder for the removal of the supraspinatus as well as the 
insertion of the gracilis flap. We observed the local anatomy for potential anastomosis of our pedicle.

RESULTS: Cadaver studies showed that the gracilis muscle resembles the supraspinatus muscle in bulk and cross-section. However, the length of the gracilis is substantially longer than the supraspinatus. Using a posterior approach to the shoulder, excision of the supraspinatus muscle and replacement with the free gracilis muscle is possible, with an anastomosis on the transverse cervical vessels and suprascapular nerve.

CONCLUSION: Using a cadaveric model, we have determined that FFMT of the gracilis muscle to replace the supraspinatus muscle in rotator cuff tear arthropathy is anatomically feasible. This represents a novel potential application for the use of a gracilis FFMT.

Learning Objectives:

- Participants will learn about progression of shoulder deformity in rotator cuff tear arthropathy.

- Participants will learn about cuff tear arthropathy treatment options.

\section{RP15 \\ AN ALGORITHMIC APPROACH TO THE MANAGEMENT OF PYOGENIC GRANULOMA: A 60-YEAR REVIEW}

\section{J Lee, H Sinno, Y Tahiri, A Perkins, M Gillardino}

PURPOSE: Pyogenic granuloma (PG) is a common benign vascular proliferation that commonly occurs on the skin. Various treatment options exist in the literature with no clear consensus. To review the literature associated with PG and their management and implement an evidence based algorithmic approach to its treatment.

METHODS: A retrospective literature review examining the treatment of cutaneous PG and their associated recurrence rates was made.

RESULTS: Thirty-four (34) manuscripts were included in our review; a total of 1162 PGs were managed using 19 different treatment modalities with 53 recurrences. Among the surgical management options, surgical excision offers the lowest overall recurrence rates (2.94\%). Of the medical treatments, cryotherapy offered the lowest overall recurrence rate (1.62\%). CONCLUSIONS: Surgical excision and primary closure should be the treatment of choice for cutaneous PGs, however if non-surgical management is undertaken cauterization with silver nitrate should be the first line treatment, although $\mathrm{CO}_{2}$ and flashlamp pulsed dye lasers are possible alternatives.

Learning Objectives:

- To review the literature associated with PG and their management.

- To discuss both surgical and non-surgical treatment options.

- To implement an evidence-based algorithmic approach to its treatment

\section{RP16}

TENDON TRANSFER OR NERVE TRANSFER FOR ELBOW FLEXION. WHAT IS THE EVIDENCE? A SYSTEMATIC REVIEW WK Ng, S Voineskos, A Thoma

PURPOSE: Both tendon transfers and nerve transfers have been reported as techniques to restore elbow flexion after upper extremity nerve injury. The purpose of this presentation is to assess the literature to determine if there is any superiority of nerve transfers over tendon transfers.

METHODS: The following electronic databases were assessed for relevant articles by two independent reviewers: Medline, Cochrane, Embase and CINAHL. Articles were selected using specific inclusion criteria. Methodological quality of observational and non-randomized studies was assessed using the MINORs scale, and a predetermined score 10 or greater was considered to indicate a high quality study. We independently extracted data for clinical outcomes from the relevant articles: range of motion, strength, quality of life, cost and complications.

RESULTS: In total, 270 articles were identified. Sixty-two articles were assessed as relevant. Our kappa agreement was 0.96. Fifty-seven of these 62 studies were retrospective and 5 were prospective. Only 11 articles were deemed as high quality using the MINORs scale. The most commonly measured outcome was strength via MRC scale (42 of 62 articles), followed by range of motion ( 18 of 62 ). Other reported factors included: time to follow-up (49 of 62 articles), injury-to-operation time interval
(41 of 62), surgical complications (33 of 62), dominant handedness/side of injury ( 9 of 62 ), and quality of life ( 5 of 62 ). No studies mentioned cost. It was not possible to accurately pool the data because investigators used many varying modalities of outcome measurement.

CONCLUSIONS: Based on the current evidence, the claim of superiority of nerve transfers vs. tendon transfers remains unsubstantiated. To better determine the overall superiority of either tendon transfers or nerve transfers, more methodologically sound randomized controlled trials are needed.

Learning Objectives:

- At the end of this presentation, the learner will be able to appreciate the variety of surgical options available for restoring elbow flexion after upper extremity nerve injury.

- At the end of this presentation, the learner will be able to list 5 different modalities of measuring outcomes after surgery for the purpose of restoring elbow flexion.

\section{RP17 \\ PERSPECTIVES ON HAND TRANSPLANTATION: KNOWLEDGE AND ATTITUDES OF AN EXPERT GROUP} A Hassa, DC Ross, CLF Temple

PURPOSE: To determine the current knowledge and attitudes of an expert group of reconstructive surgeons about upper extremity transplantation.

METHODS: An internet-based survey was generated and distributed electronically to the members of the Canadian Society of Plastic Surgery. The survey questions and four clinical vignettes served to assess respondents' demographics, background knowledge of hand transplantation and its outcomes, the risks of immunosuppression, and their attitudes and willingness to consider it as a reconstructive option. Comparative data were generated based upon respondents' answers.

RESULTS: Of the 86 surgeons responding, $79.8 \%$ had practices containing a significant portion ( $>20 \%$ ) of hand surgery. Nearly $2 / 3$ of surgeons significantly underestimated the number of upper extremity transplants completed and only $25.9 \%$ correctly identified the rate of successful transplants. The majority felt that the functional outcomes of unilateral, below-elbow transplants and prostheses were equivalent $(77.5 \%$ vs $91.1 \%, \mathrm{p}=0.028)$; however, more felt that a bilateral belowelbow transplant was superior to bilateral prostheses ( $81 \%$ vs $44 \%$, $\mathrm{p}=0.0045)$. Only a minority $(22.8 \%)$ thought the risks of immunosuppression were acceptable in unilateral amputees vs a majority $(61.5 \%)$ for bilateral amputees. However, a majority (83\%) felt that a "fully informed patient" should be able to make the decision to proceed with transplantation autonomously. Clinical vignettes revealed that the majority $(78.5 \%)$ thought the risks of non-life extending renal transplantation were acceptable whereas only $32.9 \%$ considered the risks acceptable for a unilateral upper extremity transplant $(\mathrm{p}<0.0001)$.

CONCLUSIONS: Misconceptions and biases concerning upper extremity transplantation exist even amongst expert groups and may hamper the acceptance of transplantation as a reconstructive option.

Learning Objectives:

- Recognize the limitations in knowledge regarding upper extremity transplantation.

- Discuss the challenges of expanding hand transplantation into an available reconstructive option.

\section{RP18 \\ PATIENT SATISFACTION AND RECURRENCE RATE FOLLOWING TREATMENT OF WRIST GANGLION CYSTS BY NEEDLE ASPIRATION}

G Gaudreau, M Jean, JC Lin

PURPOSE: Wrist ganglion cysts represent a frequently encountered benign tumour of the hand. Needle aspiration is a simple out-patient treatment option, but published reports suggest high recurrence rates (36-85\%). We hypothesize that high patient satisfaction with needle aspiration of ganglion cysts may account for lower than expected patient re-consultation rates, even following recurrence of the cyst. 
METHOD: All consecutive patients initially treated with needle aspiration of a ganglion cyst between October 2007 and November 2009 were included in the study. Patient data were collected prospectively. Patients were interviewed by telephone questionnaire to evaluate their satisfaction following the treatment. A short survey of practicing plastic surgeons in Quebec was also conducted, to determine the current treatment practices in use.

RESULTS: 38 patients underwent needle aspiration of a wrist ganglion cyst under the supervision of a single plastic surgeon between October 2007 and November 2009. 30 patients (78.9\%) completed a telephone questionnaire at the end of the study period. 25 (83.3\%) patients were satisfied immediately following the needle aspiration, due to partial or complete disappearance of the cyst. However, at time of follow-up (between 3 months and 2 years post-aspiration), only 12 (40\%) were satisfied with the initial treatment. No complications were reported. According to the self-reported questionnaire, 22 (73.3\%) of the patients noted a recurrence, but only $13(43.3 \%)$ had returned for a second consultation. 17 patients $(56.7 \%)$ would still choose needle aspiration as a first treatment.

CONCLUSION: Despite a high rate of recurrence of wrist ganglion cysts following needle aspiration, the complication rate is low and patient satisfaction is relatively high, suggesting that this treatment should continue to be offered to patients as a first-line option.

Learning Objectives:

- Participants will learn rates of recurrence, satisfaction and complication following needle aspiration for ganglion cyst.

\section{RP19}

\section{CLINICAL EXPERIENCE WITH THE ARTELON $®$ CMC} SPACER IN THE TREATMENT OF OSTEOARTHRITIS

\section{Q Chivers, I Macarthur, A Islur, C Murray, K Murray}

PURPOSE: Trapezial excision with tendon interposition is frequently considered the gold standard for the treatment of Trapeziometacarpal (TMC) Osteoarthritis (OA). However, the procedure is not without complications. Secondary joint collapse resulting in a decrease in stability and strength as well as pain recurrence can occur, and as such alternative treatments should be considered. The Artelon ${ }^{\circledR}$ CMC spacer is a T-shaped synthetic device consisting of a biodegradable polycaptrolactrone based polyurthaneurea which combines two methods of restoration in TMC OA through joint capsule augmentation and resurfacing of the articular surface. We present our 3 year experience using the device at our institution.

METHODS: A retrospective study of all Artelon ${ }^{\circledR}$ CMC spacer procedures conducted at the University of Manitoba between December 2007 and May 2009 was performed.

RESULTS: Fourteen patients with TMC OA received the Artelon ${ }^{\circledR}$ CMC spacer with the majority of operations (13/14) performed by a single surgeon. Ten of the fourteen patients demonstrated improvements in pain relief, strength, stability and ROM. Pain relief in four of the fourteen patients was inadequate who ultimately went on to receive trapezial excision with tendon interposition.

CONCLUSIONS: The Artelon ${ }^{\circledR}$ CMC spacer offers unique advantages over many of the existing surgical TMC OA options available for isolated Trapeziometacarpal. When performed correctly, improvements in pain relief, strength, stability and ROM can be obtained in a majority of patients while keeping the anatomy of the hand intact over time and without shortening or restricting motion of the thumb.

Learning Objectives:

- The learner will gain an appreciation for the indications for use, operative techniques, complications and expected outcomes associated with use of Artelon ${ }^{\circledR}$ CMC spacer for the treatment of trapeziometacarpal osteoarthritis.

\section{RP20}

IS IT TRUE THAT INJECTING PALMAR FINGER SKIN HURTS MORE THAN DORSAL SKIN? NEW LEVEL 1 EVIDENCE

\section{ME Wheelock, M LeBlanc, B Chung, J Williams, DH Lalonde}

PURPOSE: It has long been assumed that dorsal finger skin is less sensitive to needlestick pain than volar finger skin. Originally introduced in one of the first texts on local anesthesia written in the 1900's, this wide spread belief has resulted in the dorsal two injection technique becoming most commonly used finger block for local anesthesia. The purpose of this study was to apply a level 1 evidence producing study to find out if this is true.

METHOD: A group of 78 blinded volunteers who had the long finger of both hands poked with a $25 \mathrm{G}$ needle; one in the midline of the volar side and the other in the lateral web space of the dorsal side. Volunteers then completed a pain scale for each needle poke and ranked which technique they would prefer for future injections.

RESULTS: $\mathrm{A} \chi^{2}$ test of proportions revealed a $\mathrm{p}$ value of 0.26 . Post hoc power of test was 0.98 . There was no significant difference in pain or preference of future injection location between the dorsal and volar aspects of the finger.

CONCLUSION: The sensitivity of the dorsal vs. volar side of injection should not influence the choice of one digital nerve block technique over the other. The SIMPLE block which has one injection on the volar side is not more painful than one of the two dorsal injection finger block needles. Clearly, two dorsal needles will be more painful than a single volar injection.

Learning Objectives:

- Participants will be able to see that volar needles are no more painful than dorsal needle pokes in the finger.

- Participants will be able to realize that one volar needle poke will definitely hurt less than two dorsal needle pokes.

\section{RP21}

\section{THUMB RECONSTRUCTION: A MOBERG MODIFICATION} USING THE FIRST WEB SPACE

\section{S Thibaudeau, D Tremblay, M Tardif, A Chollet}

PURPOSE: The Moberg flap, popularized in 1965, was designed to cover soft tissue defects of the distal thumb. This neurovascular advancement flap has the advantage of conserving thumb length and sensation thus allowing for improved functional outcome. Several modifications to this technique have since been reported in the literature to allow for greater mobilization of the flap. However, these modifications carry the disadvantage of skin grafting and immobilization. We present a new modification that allows for greater mobilization of the neurovascular flap, immediate mobilization and superior functional and esthetic results.

METHODS: Since 2006, this new Moberg modification was performed at Maisonneuve-Rosemont Hospital in Montreal for the treatment of distal thumb amputations measuring less than $3 \mathrm{~cm}$. The skin of the first web space is used as a transposition flap to cover the donor site defect of the Moberg O'Brien flap followed by a z-plasty to deepen the first web space. We present three clinical cases where active range of motion of the thumb, key and three point pinch strengths and grip strength was measured within two weeks post-operatively and long-term follow-up. RESULTS: Active range of motion of the thumb, key and three point pinch strengths and grip strength were comparable to the unaffected hand in all cases. Scars were subjectively qualified as esthetically acceptable.

CONCLUSIONS: This new technique has the advantage of avoiding skin grafting which improves short term functional outcome by allowing rapid mobilization. Also, the relative lengthening of the thumb secondary to the z-plasty yields excellent esthetic and functional results. We recommend the use of this technique for distal thumb amputations measuring less than $3 \mathrm{~cm}$.

Learning Objectives:

- At the end of this presentation, the learners will have acquired the knowledge of a new modification to the Moberg O'Brien flap for distal thumb amputations. 


\section{RP22}

\section{EXPERIENCE WITH PYROHEMISPHERE ARTHROPLASTY IN CMC OSTEOARTHRITIS}

I MacArthur, Q Chivers, C Murray, A Islur, K Murray

PURPOSE: Although ligament reconstruction and tendon interposition is considered the gold standard for the treatment of basal thumb joint osteoarthritis, this treatment modality is not without complications. Joint collapse can occur secondarily with resulting pain recurrence and decreases in strength and stability. As such, alternative treatment methods should be considered. The Ascension ${ }^{\circledR}$ pyrohemisphere arthroplasty has been developed to reduce these complications, and we will present our clinical experience with this treatment.

METHODS: Twelve patients over the span of thirteen months were diagnosed clinically and radiographically with thumb CMC osteoarthritis. All received Ascension ${ }^{\circledR}$ pyrohemisphere arthroplasty by one of two surgeons. Post-operatively patients underwent splinting, physiotherapy and outpatient follow-up.

RESULTS: All patients were satisfied with pain relief, ROM, stability and strength. One patient experienced a dislocation due to failure of retention sutures. This individual underwent a revision surgery and had an uneventful recovery.

CONCLUSIONS: Despite our low sample size, this preliminary retrospective study demonstrates that pyrohemisphere arthroplasty may be an effective treatment option for patients with thumb basal joint CMC osteoarthritis.

Learning Objectives:

- Lecture attendees will understand the rationale for choosing pyrohemisphere arthroplasty for CMC osteoarthritis. Operative technique, postoperative care and complication management will also be discussed.

\section{RP23}

\section{THE EFFECT OF PERI-OPERATIVE ANESTHESIA \& ANALGESIA} METHODS ON PAIN AND FUNCTIONAL RECOVERY AFTER HAND INJURIES - RETROSPECTIVE REVIEW

\section{O Fouda-Neel}

PURPOSE: Chronic pain is debilitating and is a major cause of disability to patients. Adverse life style effect as well as the big economic burden on organizations due to absence from work warrants a better way to optimize pain control for the hand injury patients.

METHODS: Retrospective review of 450 patients followed up during a six months period in a regional tertiary Hand center, analysis of the presenting complaint, type of analgesia, surgical interventional and intra operative anaesthesia method was made. The long-term pain score outcomes were analyzed.

Premorbid hand condition and duration of immobilization were entertained to look for co-factors affecting final outcome. Total No. of post operative visits and total duration of occupational therapy as well as time taken off work was noted.

RESULTS: Of the 450 patients, $5.2 \%$ suffered from severe pain of $>1$ month duration post operative requiring referral to pain service center, $6 \%$ had moderate pain that resolved within a month (average 4 post operative visits) and $89 \%$ had mid pain that resolved at the 1 st post operative visit.

Pain score following each type of anesthesia and analgesia used is presented. No statistical significant difference between pain score in populations treated by regional block versus a general anaesthesia nor between those who received Opioids versus Gabapentin's or Nsaids Analgesics was noted.

CONCLUSION: A database using the aforementioned criteria was created for our center. Although no statistical significant difference between our treatment groups was found, this study could serve as a ground for a future prospective trial comparing pain scores using well documented pain score tools to better compare those treatment groups in a search for the best possible pain outcome after hand injuries.

Learning Objectives:

At the end of this presentation the participant will:
- Understand the referral pattern and distribution of patients to a tertiary care Hand center.

- Understand that different modalities of anaesthesia in the operating room or analgesia postoperatively could have different effects on the post operative pain course of the patients.

- Understand that a good percentage of hand injury patients could have debilitating pain from their injury and that this could lead to chronic disability and even loss of jobs.

\section{RP24}

\section{CAFFEINE RESTRICTION IN MICROSURGERY: 2010 SURVEY} OF CANADIAN CENTRES

\section{DT Tang, JG Williams, DH Lalonde}

PURPOSE: Microsurgical procedures are routine practice for many Canadian Plastic Surgeons. A typical component of postoperative care is dietary and pharmacologic caffeine restriction. Despite a paucity of evidence in the literature, this practice has been implemented with the intention of avoiding the theoretical risk of flap failure from vasoconstriction of the microvascular pedicle from exogenous caffeine. This project is designed to gauge the prevalence of postoperative dietary and pharmacologic caffeine restriction in Canadian microsurgical centres, and identify the basis for this practice.

METHOD: After a thorough review of the literature through PubMed, a 10 question survey was developed and distributed to practicing Plastic and Reconstructive Surgeons in Canada. The survey was distributed through the Dalhousie University online survey tool "Opinio" to all members of the Canadian Society of Plastic Surgeons member directory.

RESULTS: Demographic statistics were calculated. Numbers were tallied with respect to forms of caffeine restriction employed and levels of evidence used to support surgeon practice. We then examined relationships between participant practice type, microsurgical experience, and the use of caffeine restriction. Comparison across groups was done using the ANOVA statistical test.

CONCLUSIONS: Across a variety of clinical practice types and microsurgical experience, exogenous caffeine restriction in the postoperative management of microsurgical patients is a common practice. There is no clear, unifying evidence to support this practice. This survey serves as the first step in developing a proper randomized controlled trial to determine the utility of caffeine restriction in postoperative microsurgery patients.

Learning Objectives:

- At the end of this presentation, the learner will critically analyze their current microsurgical practice as it relates to postoperative exogenous caffeine restriction in light of little scientific evidence.

\section{RP25}

EVALUATING THE ROLE OF PHOTOTHERAPY IN WOUND HEALING

D Rizis, JP Brutus, S Cugno, S Rastogi, A Nikolis

PURPOSE: Numerous studies evaluating laser and light technologies on wound healing have been published. Low-level laser light therapy (LLLT), light emitting diode (LED) phototherapy and photodynamic therapy (PDT) have been used in the fields of ophthalmology, dermatology and dental surgery, but are seldom included in the plastic surgeon's armamentarium in wound healing.

METHODS: A complete review of the literature was performed using the following key words: "low-level laser light therapy, light emitting diode phototherapy, photodynamic therapy, phototherapy, wound repair, and wound healing", from the Medline, Ovid and Cochrane online databases. Articles were screened, coded and reviewed in an attempt to categorize and highlight key effects of phototherapy in wound healing.

RESULTS: Wavelengths of 633, 660, 780, and 890nm at energy densities of 1.3 to $4.71 \mathrm{~J} / \mathrm{cm}^{2}$ were shown to have positive local effects on wounds, through fibroblast proliferation, mitochondrial respiration, ATP synthesis, as well as promoting collagen deposition and neovascularization. The use of phototherapy in animal wound healing models has 
shown promising results. To date there are few studies evaluating this technology in human wounds.

CONCLUSION: Published literature evaluating the impact of phototherapy and wound healing demonstrates numerous interactions between light therapy and specific aspects of wound healing. Difficulty in data interpretation arises from the use of different wound healing models and different outcome measures. Photodynamic therapy is an important adjunct in wound healing leading with a significant impact on chronic or stagnating wounds. Based on animal and basic science data, the role of wavelength specific light therapy needs to be reevaluated in human wound healing.

Learning Objectives:

- To identify and categorize the various modalities of light therapy currently in use.

- To acknowledge the prospective use of phototherapy in promoting wound repair.

\section{RP26 \\ THE USE OF SCIATIC NERVE TISSUE EXPANSION IN THE TREATMENT OF SEVERE KNEE FLEXION ANOMALY. A CASE OF POPLITEAL PTERYGIUM SYNDROME}

\section{G Al-Thubaiti, L Ross}

BACKGROUND: With less than 100 cases reported, popliteal Pterygium syndrome (PPS) is very rare. Features include cleft lip/palate, lip pits, syndactyly, genital anomalies and the popliteal pterygium with knee flexion deformity. Severe skin webbing, an aberrant musculotendonous component from calcaneus to ischium and foreshortening of the sciatic nerve make management challenging. Serial casting, surgical release, femoral osteotomy and shortening, external fixators, and one suboptimal case of a tissue expander in an older child has been reported.

METHODS: A 4 month old female was diagnosed with PPS in our centre. She had bilateral severe pterygium that extended from heel to ischium with severe knee flexion contracture bilaterally. The goal of treatment was for her to reach age appropriate motor milestones. At age 6.5 months we utilized wide dissection and excision of the aberrant musculotendonous unit, achilles lengthening, fasciocutaneous Z-plasties and right sided tissue expansion to resolve the severe shortening of the sciatic nerve. The tissue expander was removed at 9 months of age.

RESULTS: The sciatic nerve and skin expansion was enough to allow full extension of the right knee following soft tissue release with no short or long term motor complications. She was walking unassisted at 15 months. Two years after surgery, the patient is able to extend her knees fully, with normal sciatic nerve function, and has not needed any further procedures.

CONCLUSION: Sciatic nerve tissue expansion is a valuable option to correct PPS webs.

Learning Objectives:

- Report a popliteal pterygium syndrome case.

- Report our experience with the use of early tissue expanders as a method to treat severe pterygium while avoiding leg shortening procedures.

\section{RP27}

\section{AN INNOVATIVE NIPPLE RECONSTRUCTION TECHNIQUE USING DERMAL FLAPS AND CARTILAGE GRAFT}

\section{Lyons, PS Chang}

PURPOSE: In nipple reconstruction, the loss of nipple projection postoperatively is often a concern for both the patient and surgeon. The use of dermal flaps was first described by Hartramptf and Culbertson in 1984. By combining the use of dermal flaps and autologous cartilage, we present an innovative technique for nipple reconstruction in the reconstructed breast.

METHOD: After marking of the proposed nipple location and infiltration of local anesthestic, three elliptical areas at the four, eight and 12 o'clock positions are deepithelialized. The distal halves of these sections are then raised. A cartilage graft is secured in the subcutaneous tissue through to the skin with a 4-0 Prolene suture. Using two Keith needles and a 2-0 PDS, the distal ends of the dermal flaps are turned underneath their proximal portions and secured by passing the suture through the skin opposite to the ellipse. These flaps are used to increase and support the projection of the cartilage graft. A periareolar intradermal 2-0 PDS is then placed around the eventual areola to pull the skin together. The elliptical donor sites are closed primarily with deep dermal 3-0 Vicryl and horizontal mattress 5-0 plain gut suture.

RESULTS: Using this technique, we are able to provide additional support for the cartilage graft and allow for enhanced projection of the nipple postoperatively. Although there are additional scars from the donor sites, this can often be camouflaged by subsequent areolar tattooing.

CONCLUSIONS: By using dermal flaps to support the cartilage graft, this technique aids the surgeon in combating the often inevitable loss of projection of the nipple after reconstruction.

Learning Objectives:

- Our colleagues will have learned an innovative nipple reconstruction option that they may apply in their own practices. 


\title{
Groupe pour L'Avancement de la Microchirurgie Canada (GAM)
}

\author{
Abstracts presented at the \\ 31st Annual Meeting / 31 $\mathrm{e}$ Reunion annuele \\ June 16, 2010
}

01

\section{FUNCTIONAL, PSYCHOLOGICAL AND QUALITY OF LIFE OUTCOMES AFTER MANDIBULAR RECONSTRUCTION FOR OSTEORADIONECROSIS WITH OSSEOCUTANEOUS FREE FLAPS IN ORAL CANCER PATIENTS}

C Payne, R Gilbert, G Devins, D Goldstein, J Ringash, $\underline{\text { SOP Hofer }}$

PURPOSE: The objective was to compare the functional, psychological and disease-specific quality of life (QOL) outcomes in oral cancer patients following conservative vs. surgical treatment with resection/ microvascular reconstruction.

METHODS: Oral cancer patients at the Princess Margaret Hospital between January 1999-2009 provided data. Two ORN treatment groups: ORN surgical group 1 - resection/microvascular mandibular reconstruction and ORN conservative group 3 - ORN with conservative management were matched on age, sex and time since radiation with two non-ORN groups: Non-ORN surgical group 2 - surgical excision, mandibular reconstruction/radiation and non-ORN group 4 - radiation alone. One time self-report surveys included: the CES - Depression Scale (emotional distress); Affect Balance Scale (psychological well-being); and the Atkinson Life Happiness Rating Scale. Treatment-related concerns were measured using Andrykowski's "Back to Normal" and a Fear-of-Cancer-Recurrence item and QOL using the EORTC - HN37.

RESULTS: A multivariate analysis of covariance was performed comparing the 4 matched groups and controlling for empirically identified covariates. ORN surgical group 1 and non-ORN surgical group 2 reported significantly higher subjective wellbeing as compared to the non-surgically treated ORN conservative group $3(\mathrm{p}=0.02)$; due largely to depressive symptoms. ORN groups $1 \& 3$ reported significantly more fear of cancer recurrence and concern about returning to normal compared to the non-ORN group $4(\mathrm{p}=0.02)$. Controlling for treatment related side effects disease-specific QOL (social eating, contact and sexuality) did not differ significantly across the groups.

CONCLUSIONS: Although mandibular reconstruction for ORN is an effective treatment, its impact on QOL is not uniform across procedures. Surgically managed groups reported greater subjective wellbeing, despite more invasive treatment, yet both ORN groups reported greater fear of recurrence and concern about returning to normal.

Learning Objectives:

- Evaluations of QOL outcomes in head and neck cancer should involve a broad range of outcomes, beyond disease-specific health-related QOL to avoid overlooking important, but subtle effects.

\section{2}

UNION AND BONE RESORPTION OF FREE FIBULAR FLAPS FOR MANDIBULAR RECONSTRUCTION

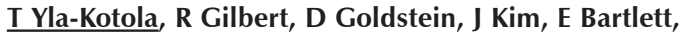

SOP Hofer

PURPOSE: The purpose of this study is to evaluate union rates and bone resorption after free fibular flap reconstruction for mandibular reconstruction and to study which factors contribute to the healing of the neomandible.

METHODS: 133 patients who required mandibular resection and reconstruction with a fibular flap between 2000-2009 were included into the study. Clinical data for patients' age, gender, indication for surgery and history of radiotherapy was collected as well as the surgical data for location and size of the defect, number and type of osteotomies required and type of plates used. We assessed the CT scan images taken at six months and one year postoperatively to evaluate union of the neomandible. CT images taken before radiotherapy and after completing the radiotherapy were assessed for the fibular bone resorption rates in terms of the change in vertical height. Statistical analysis was done to evaluate which factors influenced the healing of the neomandible.

RESULTS: 104 patients had malignant disease, most often being squamous cell carcinoma (71\%). Mean age was 57 years (14 to 88y), 82 patients received radiotherapy. 39 minor complications consisted of infections, seromas, hematomas or fistulas. 16 major complications consisted of partial or total flap failures or events requiring an operative procedure due to a complication. Preliminary CT results show that most of the fibulas healed well, and bone resorption seems to be present especially after radiotherapy. Union rates and the amount of bone resorption will be reported after completing the analysis.

CONCLUSIONS: After final analysis of the data we will have longterm information on the rates and predictors of bony healing after mandibular reconstruction with free fibular flaps.

Learning Objective:

- This study will show us how the neomandible is healing after free fibular flap reconstruction.

\section{3}

RECONSTRUCTION OF THE PAEDIATRIC MANDIBLE WITH FREE OSSEOUS TRANSFER (LITERATURE REVIEW AND CASE PRESENTATION OF GUNSHOT TO THE FACE IN AN 11-YEAR-OLD BOY)

A Perkins, H Sinno, L Lessard

PURPOSE: We encountered an 11 year old boy who suffered a shotgun injury to his right face with almost complete obliteration of the mandible. Our purpose was to evaluate the potential for growth of the reconstructed mandible in the paediatric population (from free osseous transfer) in order to correctly design the size and shape.

METHOD: Medline search of mandible reconstruction in the paediatric population using free osseous transfer and also the ethnic variations in the shape of the mandible.

RESULTS: Only five case series were found and only one showed radiological quantifiable evidence showing partially restricted growth of the reconstructed mandible from free osseous tissue transfer. There were ethnic differences in mandible size and shape.

CONCLUSIONS: A paucity of information exists on the reconstructed mandible's growth capacity in the paediatric population. We designed our mandible to be slightly larger than an age, height and sexadjusted mandible (1:1 axial CT Facial Bones printout of a similar patient onto a plain film as a template) in order to compensate for a future-restricted growth in reference to the rest of the facial bones and also adjusted for ethnic differences in the shape of the mandible.

Learning Objectives:

- Participants will be able to provide a comprehensive review of the literature on paediatric mandible reconstruction.

- Participants will be able to explain our rationale and our technique for reconstructing the form and shape of the paediatric mandible.

- Participants will be able to describe the growth of the mandible and ethnic variations of mandible shape. 
04

\section{A NEW TECHNIQUE FOR TOTAL ORAL SPHINCTER RECONSTRUCTION FOLLOWING PERIORAL BURN INJURY}

M Choi, L Sigurdson, G Althubaiti, S Logsetty, T Hayakawa

PURPOSE: We describe our experience reconstructing a full-thickness perioral burn injury using a split-gracilis functional muscle free tissue transfer lined with skin graft and facial artery myomucosal (FAMM) flaps. To our knowledge this is the first report of this method of reconstruction.

METHOD: A healthy young male sustained a severe facial perioral contact burn from an electrical wire, obliterating $80 \%$ of the lower lip and $60 \%$ of the upper lip. After resuscitation and debridement, a functional gracilis free-tissue transfer was performed. Intrafascicular dissection of the obturator nerve was performed with intra-operative nerve stimulation to identify two separate fascicular groups innervating two separate muscle units of the gracilis. One neuromuscular unit was used to reconstruct the upper lip with neurorrhaphy to a buccal branch of the facial nerve; the other unit was used to reconstruct the lower lip with neurorrhaphy to the marginal mandibular branch. The muscle strips were left attached at both ends and reinforced to the modiolus bilaterally to complete the total sphincter reconstruction. The muscle was lined with FAMM flaps to reconstruct effective buccal sulci and create a wet vermillion, with skin grafting to form an outer dry vermillion.

RESULTS: The wounds healed without complication. Both gracilis strips showed signs of reinnervation within 3 months. Sphincter activity has been reestablished. Our patient was able to purse his lips and eat regular food with utensils, without drooling.

CONCLUSIONS: The innervated gracilis can provide functioning muscle for orbicularis reconstruction. It can be lined with skin grafts and mucosal flaps to restore form and function and can be split with an intrafascicular and intramuscular dissection to create a functional sphincter.

Learning Objective:

- Describe the split-gracilis free functional muscle transfer.

\section{5}

\section{MANAGEMENT OF THE EXPOSED, INFECTED CRANIOPLASTY WITH FREE TISSUE TRANSFER AND CUSTOM ALLOPLASTIC IMPLANTS}

\section{FM Yau, S Williamson}

PURPOSE: Management of the exposed, infected cranioplasty confers a complex reconstructive challenge as reconstruction of the soft tissue deficit often occurs in a wound bed compromised by scarring, infection and radiation changes. The authors report the use of free tissue transfer and custom designed polyetheretherketone implants as a safe and reliable treatment option.

METHODS: Three patients with exposed primary cranioplasty were retrospectively reviewed between 2007 and 2009. Surgical technique and reconstructive details of the two-staged approach were assessed, including concepts of flap selection and design. Patient demographics, underlying cause, defect size, complications and implant selection were reviewed.

RESULTS: All patients (age 52 to 79 ) had composite defects with the bony component ranging in size from 12.5 to $28.8 \mathrm{~cm}^{2}$ (mean $78.8 \mathrm{~cm}^{2}$ ). All patients presented on a delayed basis with a history of prior or current infection of the affected area. Defects were due to trauma requiring craniectomy or following tumor resection. All patients underwent removal of the primary cranioplasty with concomitant free tissue transfer. Custom polyetheretherketone implants were fabricated to restore skull contour at a second stage following establishment of a viable wound bed. No recurrence of infection occurred. Final scalp contour and functional outcome were excellent.

CONCLUSION: The combination of free tissue transfer and custom alloplastic implants is a reasonable treatment option in the management of infected cranioplasty. This approach has been effective in the eradication of infection while providing stable skeletal support and soft tissue coverage with minimal donor morbidity.

Learning Objectives:

After the presentation, the viewer will be able to:

- Define the indications and timing for secondary cranioplasty.

- Understand the surgical options and principles for reconstructing the cranium and overlying soft-tissue deficits.

- Apply this two-staged approach in the clinical setting for management of the patient with infected cranioplasty.

\section{6}

\section{A COMPARISON OF MICROSURGICAL EDUCATIONAL PARADIGMS USING AN OBJECTIVE STRUCTURED ASSESSMENT OF TECHNICAL SKILLS}

\section{P Rasmussen, K Almutairi, S Morris}

PURPOSE: Despite the importance of precise technique in microsurgery, little research has been conducted to determine the most efficacious method of training surgical residents. In this study, senior medical students were taught to perform microvascular anastomoses, either by watching a series of conventional educational videos, or by one-on-one instruction from a microsurgical technician in a skills laboratory. Using a fresh turkey thigh model, performance was assessed with a microsurgical objective structured assessment of technical skills (MOSATS).

METHODS: A total of 26 medical students at Dalhousie Medical School were randomized to two groups: a self-directed group and a hands-on group. The self-directed group watched four hours of instructional videos, while the hands-on group received four hours of one-on-one teaching in a skills laboratory. After their initial instruction, all participants had the opportunity to practice their technique in a skills laboratory for four hours. Following this practice, each participant was filmed performing a complete anastomosis. These recordings were reviewed and scored by a panel of plastic surgeons, using the MOSATS rating tool. The MOSATS tool includes three measures of assessment: duration of anastomosis, a checklist of behaviours important in microsuturing, as well as a Global Performance Rating.

RESULTS: While the checklist and GPR scores of the self-directed and hands-on groups were not significantly different, the hands-on group was able to perform anastomoses faster than the self-directed group $(\mathrm{p}=0.0385)$.

CONCLUSIONS: Given that time can be a critical factor in determining the outcome of microsurgical procedures, the increased speed with which students who received hands-on training could perform anastomoses suggests that this instructional method may be beneficial.

Learning Objective:

- Be aware of advantages and disadvantages of self-directed versus hands-on training.

\section{7}

\section{ANATOMIC ANALYSIS OF THE INTERNAL MAMMARY ARTERY FOR USE IN CORONARY REVASCULARIZATION: WHAT IS THE MINIMUM LENGTH REQUIRED? A CADAVERIC STUDY}

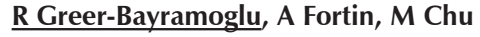

PURPOSE: The number of autologous breast reconstructions being performed with a preference for the internal mammary artery (IMA) has dramatically increased over the past two decades. No study has yet to examine the possible decreased lifespan this may cause when the IMA is no longer available for use in cardiac revascularization.

METHODS: An anatomic study on ten preserved female cadavers was performed to examine variations in the length of the IMA in relation to the 3rd, 4th and 5th intercostal spaces (ICS). Cadaver height, sternal length, chest circumference, and width between ICS were recorded. The minimal length of IMA required to reach a predetermined anastomotic point on the left anterior descending (LAD) coronary artery was determined.

RESULTS: The mean length for the IMA to reach the LAD on the left was $10.0 \mathrm{~cm}$, with the mean length to the left $3 \mathrm{rd}$, 4th and 5 th ICS at 
$8.2 \mathrm{~cm}, 11.3 \mathrm{~cm}, 13.7 \mathrm{~cm}$, respectively. On the right the mean length to the LAD was $12.0 \mathrm{~cm}$ and lengths to $3 \mathrm{rd}$, 4th and 5 th ICS being $7.6 \mathrm{~cm}$, $10.7 \mathrm{~cm}, 13.2 \mathrm{~cm}$.

CONCLUSION: The left IMA was sufficient in length to reach the anastomotic point on the LAD in all the cadavers when taken at the 4th ICS. There was more variability on the right. The authors recommend that during autologous breast reconstruction, the left IMA anastomosis occur at or below the 4th ICS to improve patient survival should future cardiac revascularization be required.

Learning Objective:

- Participants will change their clinical practice when performing autologous breast reconstructions, to maintain the option of future cardiac revascularization using the IMA.

\section{8}

\section{PATIENT REPORTED SATISFACTION WITH BREAST RECONSTRUCTION WITH TRANSVERSE UPPER GRACILIS FLAPS}

G Althubaiti, M Choi, L Sigurdson, T Hayakawa, E Buchel

BACKGROUND: We have previously reported high complication rates following Transverse Upper Gracilis (TUG) free flaps breast reconstructions. Patient acceptance of the procedure may however be independent of the complications found. The purpose of the study was to determine patient satisfaction with TUG flap breast reconstructions using a validated breast reconstruction specific outcome tool.

METHODS: Sixty one TUG flaps were performed on 37 patients. All patients were contacted and sent the Breast-Q( $\bigcirc$ breast reconstruction outcomes questionnaire (Memorial Sloan Kettering, 2007) and an additional satisfaction questionnaire.

RESULTS: The initial response rate to date has been $40 \%$ after the first mail-out. Of the 14 patients responding 13 were satisfied with their reconstruction and would recommend the procedure. The remaining patient was unhappy as a result of persistent lower leg lymphedema.

Of the satisfied patients six reported concerns with donor leg lymphedema. Sexuality was affected to a variable degree among patients, with two patients reporting improved sexuality following their thigh surgery. Six patients were unhappy with their appearance in bathing suits. A full report will be presented.

CONCLUSION: Despite having a higher complication rate than other methods of breast reconstruction, the TUG free flap shows a high degree of patient satisfaction in our series.

Learning Objective:

- Patients who undergo breast reconstruction with TUG flaps reported high satisfaction rate.

\section{9}

\section{IMMEDIATE POST-MASTECTOMY RECONSTRUCTION IS ASSOCIATED WITH IMPROVED BREAST CANCER-SPECIFIC SURVIVAL: EVIDENCE AND NEW CHALLENGES FROM THE SURVEILLANCE, EPIDEMIOLOGY, AND END RESULTS DATABASE}

M Bezuhly, C Temple, LJ Sigurdson, RB Davis, G Flowerdew,

EF Cook Jr

PURPOSE: Although immediate breast reconstruction is increasingly offered as part of post-mastectomy psychosocial rehabilitation, concerns remain that it may delay adjuvant therapy or impair detection of local recurrence. No single population-based study has examined the relationship between immediate breast reconstruction and breast cancer-specific survival.

METHODS: Using data from the US National Cancer Institute's Surveillance, Epidemiology, and End Results (SEER) registries, breast cancer-specific survival was compared for female unilateral mastectomy patients who did or did not undergo immediate breast reconstruction using Cox proportional hazards models. Models were fitted adjusting for known demographic and disease severity variables, and stratifying on reconstruction type (implant or autologous) and age.
RESULTS: Improved breast cancer-specific survival was observed among all immediate breast reconstruction patients compared to patients who underwent mastectomy alone (hazard ratio $[\mathrm{HR}]=0.74$; $95 \%$ CI, 0.68 to 0.80 ). Implant reconstruction patients below 50 years of age demonstrated the greatest apparent survival benefit ( $\mathrm{HR}=0.47 ; 95 \%$ CI 0.28 to 0.80 ). Similarly, autologous reconstruction was associated with improved cancer-specific survival among patients below the age of $50(\mathrm{HR}=0.58 ; 95 \% \mathrm{CI} 0.42$ to 0.80$)$ and between ages 50 to 69 (HR $=0.61 ; 95 \%$ CI 0.43 to 0.85 ).

CONCLUSION: Immediate breast reconstruction is associated with decreased breast cancer-specific mortality, particularly among younger women. We believe this association is more likely attributable to imbalances in socioeconomic factors and access to care than to inadequate adjustment for tumor characteristics and disease severity. Further research is needed to identify additional prognostic factors responsible for the improved cancer survival among women undergoing immediate post-mastectomy reconstruction.

Learning Objective:

- To understand the association between immediate breast reconstruction and breast cancer-specific survival in a large population-based study.

- To recognize the different apparent beneficial associations between autologous and alloplastic reconstruction and breast cancer-specific survival.

- To appreciate inherent limitations and strengths of retrospective registry data in making causal inferences and generating hypotheses, respectively.

\section{0}

\section{THE SGAP FLAP IN BREAST RECONSTRUCTION - BACKUP OR FIRST CHOICE?}

\section{AA Yaghoubian, B Boyd}

BACKGROUND: Breast reconstruction using autologous tissue provides a durable reconstruction with minimal donor site morbidity. Typically, either the deep inferior epigastric perforator flap (DIEP) or the transverse rectus abdominis myocutaneous (TRAM) flap is first choice for breast reconstruction. The superior gluteal artery perforator (SGAP) flap is often chosen by the surgeon when DIEP/superior inferior epigastric artery (SIEA)/TRAM flaps are contraindicated. The purpose of this study is to investigate whether the SGAP flap ought to be considered as a primary modality rather than as a backup flap for breast reconstruction.

METHODS: A retrospective chart review of all consecutive unilateral breast reconstructions using either the DIEP/SGAP flap by a single surgeon between 2006 and 2009 at a community hospital. Data collected included age, gender, history of abdominoplasty/previous flap, length of hospital stay and surgery, amount of blood loss, need for blood transfusion during the operation, the type of anastomosis, whether the reconstruction was delayed or immediate, and the length of follow-up. Outcome measures were flap survival and complications.

RESULTS: Over the five year study period, 16 SGAP and 24 unilateral DIEP flaps were performed. No flaps were lost. A comparative analysis was performed (Table).

\begin{tabular}{lrrc} 
Variable & SGAP $(\mathbf{n}=\mathbf{1 6})$ & DIEP $(\mathbf{n}=\mathbf{2 4})$ & P value \\
\hline Median age & 54 & 49 & 0.4 \\
Median LOS (days) & 5 & 5 & 0.5 \\
Previous flap & 5 & 0 & 0.007 \\
Previous abdominoplasty & 3 & 0 & 0.06 \\
OR time (hrs) & 9 & 7.9 & 0.007 \\
Median EBL & 300 & 252.5 & 0.2 \\
Immediate reconstruction & 7 & 12 & 0.8 \\
Complication rate & 3 & 2 & 0.4 \\
Transfusion & 5 & 4 & 0.4 \\
Median \# of units & 1 & 1 & 1 \\
Need to go back to OR & 1 & 1 & 1 \\
Flap survival & $100 \%$ & $100 \%$ & 1 \\
Median follow up (days) & 311 & 360.5 & 0.3 \\
\hline
\end{tabular}

CONCLUSION: The SGAP flap is typically chosen as an alternative for breast reconstruction when DIEP/SIEA/TRAM flaps are contraindicated. Although the operative time is one hour longer, the findings of 
the study suggest that it can achieve similar favorable outcomes as DIEP flaps without increasing patient morbidity and mortality. The SGAP flap should be considered as the primary modality of breast reconstruction more often.

Learning Objectives:

- To compare the SGAP flap with the DIEP flap in terms of morbidity, and outcome.

- To evaluate the SGAP flap as a primary rather than a secondary reconstructive modality.

- To enumerate the benefits of SGAP breast reconstruction.

\section{1}

\section{AN APPROACH TO BREAST MICROSURGERY USING AN ESTABLISHED FOUR-COMPONENT TRAINING SCHEME THAT DRAMATICALLY IMPROVES EFFICIENCY}

\section{K Boyd, K Shokrollahi, N Guay}

PURPOSE: Increased efficiency in the operating room has substantial benefits, including decreased patient morbidity and mortality, decreased wait list times, and decreased surgeon morbidity. In a health care environment where access to resources is limited, approaching microsurgery with an established routine dramatically improves efficiency. The purpose of this paper is to share our approach to single-stage, unilateral, free autologous breast reconstruction.

METHODS: We have developed a training scheme to improve operative efficiency that divides single-stage, unilateral free autologous breast reconstruction into four components (recipient vessel preparation, flap harvest, microsurgery, and artistry). Each component is further subdivided into six sequential steps.

RESULTS: Between 2006 and 2009, this method has been employed by the senior author in 174 patients presenting for free autologous breast reconstruction. Successful single-stage reconstruction was achieved in $75.9 \%$ of patients. In the last 40 flaps, the average time from patient entry into the operating theatre to exit to recovery room was 3 hours 46 minutes. Only two of the 40 patients required a second general anaesthetic.

CONCLUSIONS: We have established a routine approach to singlestage, unilateral free autologous breast reconstruction that results in improved operative flow. This training scheme allows surgeons and trainees to highlight areas for potential improvement. This approach is reproducible and straight-forward, and has resulted in decreased operating times, increased efficiency, and as a result shorter wait times.

Learning Objectives:

- To understand the benefits of operating room efficiency for patients, surgeons, and hospitals and the role of a four-component training scheme in achieving efficiency in the OR.

- To learn our strategy for single stage, unilateral free autologous breast reconstruction.

\section{2}

VENOUS THROMBOEMBOLISM FOLLOWING MICROSURGICAL BREAST RECONSTRUCTION: AN OBJECTIVE ANALYSIS IN 225 CONSECUTIVE PATIENTS USING LOW-MOLECULAR-WEIGHT HEPARIN

\section{PROPHYLAXIS}

V Lemaine, C McCarthy, B Mehrara, A Pusic, PG Cordeiro, JJ Disa

\section{3}

\section{DEVELOPMENT OF A BREAST RECONSTRUCTION SATISFACTION QUESTIONNAIRE (BRECON) PART II: ITEM REDUCTION \\ C Temple, EF Cook, DC Ross, M Bettger-Hahn, J MacDermid} OBJECTIVE: To develop a questionnaire to assess women's satisfaction with breast reconstruction.

METHODS: Item generation: Items were previously generated from Part I of this study. Four focus groups ( 20 women) generated 515 items, ten experts developed 171 items, and literature review produced
227 items. These 913 potential items were reduced to 183 by eliminating redundancy. These items were stratified into 14 themes, ranging from preoperative expectations and process of care to donor site problems, impact on relationships, and overall satisfaction.

ITEM REDUCTION: These 183 items were formally reduced by calculating item impact scores. To derive the impact scores, a questionnaire was mailed out to reconstructed women and experts. Participants were required to rate each item for importance and frequency. Using the "group-value" method, the overall item-impact score was derived as the product of the average importance and average frequency score. Intraclass correlation coefficient (ICC) was used to determine the similarities between patient and expert impact scores. The highest patient impact questions were maintained for Part III of this study.

RESULTS: Thirty-two of 40 reconstructed women and 19 of 19 experts responded to the questionnaire mail-out. ICC between patients and experts was 0.71 [0.62 0.77], indicating "good" but not "excellent" agreement, reinforcing the importance of patient involvement in questionnaire development. Seventy-seven items of the women's top 100 also made the experts' top 100 list. Women rated abdominal donor site issues higher than experts, and experts rated breast softness and symmetry higher than women.

CONCLUSION: A 100-item pilot questionnaire for breast reconstruction satisfaction was developed for psychometric testing.

Learning Objective:

- To understand the methodology used for item reduction in questionnaire development.

\section{4}

CHOOSING THE IDEAL FLAP FOR SOFT TISSUE COVERAGE OF DISTAL FOREARM AND DORSAL HAND

B Parrett, J Prince, RF Buntic, GM Buncke, B Safa, D Brooks, Lou-Merhi

BACKGROUND: Distal forearm and dorsal hand soft tissue defects remain very challenging to reconstruct. It is no longer satisfactory to just cover a defect without any concern for aesthetics of the donor and recipient sites. There is no comparative large series available in the literature demonstrating the superiority of one type of flap in soft tissue coverage of distal forearm and dorsal hand.

PURPOSE: Try to find the ideal donor site for soft tissue coverage of distal forearm and dorsal hand, taking into account extent of the injury, recipient site's requirements, donor site morbidity and patient's body habitus.

METHODS: A five year retrospective chart review was undertaken (2004-2008); it included patients with crush avulsion injury to distal forearm / dorsal hand and underwent free tissue coverage. Recipient site's requirements in terms of type of tissue needed, contour/color match, and the required flap pedicle length/diameter, were analyzed. Donor site morbidity was also reviewed in terms of contour changes, visible scar and functional loss.

RESULTS: 121 patients (age range, 16-79) were included. Out of 121 free flaps performed, there were 46 fasciocutaneous flaps, 53 muscle flaps, 19 fascial flaps and 3 venous flaps. Total flap failure occurred in 2 patients and partial flap loss in 2 patients. Donor site hematoma occurred in 3 cases. Flap debulking procedures were performed in 26 out of 121 cases $(21.5 \%)$.

CONCLUSION: In the era of multiple flap options available, there is no ideal flap to distal forearm and dorsal hand. Flap selection must be individualized to fit the indications specific to each patient and to every single defect. A thin fascial flap with a sheet skin graft is a good option but the aesthetic outcome is still unpredictable because of the skin graft healing factor.

A venous flap is another excellent option with its increased versatility. Fasciocutaneous flaps tend to be bulky even in the thinnest patient.

Learning Objectives:

- Review the principles of distal upper extremity soft tissue reconstruction.

- Review the different types of free flaps available for reconstruction of the distal upper extremity. 
15

\section{USE OF ARTERIALIZED SAPHENOUS VEIN VENOUS FLOW- THROUGH FLAPS AS A TEMPORIZING MEASURE FOR HAND SALVAGE IN CONTAMINATED WOUNDS: A CASE SERIES}

A Islur, B Safa, G Buncke, R Buntic, D Brooks

INTRODUCTION: Definitive soft tissue reconstruction in the face of a contaminated "dirty" wound is contraindicated. This problem is further complicated when such a circumstance is associated with limb ischemia. Traditional vein grafts, without vascularized soft tissue coverage, in this scenario are associated with desiccation, blow-out, infection and early clotting leading to limb loss. The use of an arterialized interpositional saphenous vein venous flow-through flap (VFTF) is a novel technique which avoids such complications and temporizes the wound until definitive coverage can be safely obtained.

METHODS: We present 4 cases of acute/sub-acute ischemia of the hand following traumatic crush-avulsion injuries to the upper extremity. All wounds were considered clinically contaminated or dirty. All patients were revascularized with an arterialized interpositional saphenous vein venous flow-through flap (VFTF).

RESULTS: VFTF size ranged from $3 \mathrm{~cm}$ wide $\times 10 \mathrm{~cm}$ long to $3 \mathrm{~cm} \times$ $18 \mathrm{~cm}$. All flaps were anastomosed to the radial artery proximally and either the radial artery or the superficial palmar arch distally. All hands were successfully revascularized and all VFTF survived. Subsequent debridements of all wounds were performed until definitive coverage could be obtained with a latissumus dorsi $(n=3)$ or rectus abdominus free flap $(n=1)$ 4-7 days following revascularization. No complications occurred. At the time of free flap coverage, the cutaneous portion of the VFTF was removed. All limbs remained perfused following discharge from hospital. CONCLUSION: We present the first documented case series of arterialized saphenous vein VFTF for upper extremity salvage. VFTFs represent a novel technique in revascularizing a contaminated ischemic limb when vascularized soft tissue wound coverage cannot be obtained over top of traditional vein grafts.

Learning Objectives:

- The audience will understand the indications for using an arterialized saphenous vein venous flow-through flap.

\section{6}

\section{DISTAL RADIUS RECONSTRUCTION WITH VASCULARISED FREE PROXIMAL FIBULA FLAP: A CASE REPORT AND LITERATURE REVIEW}

\section{Lutfy, O Fouda neel, H Sinno, M Luc}

PURPOSE: Excision of the distal radius for the treatment of osteosarcoma in children has two main reconstructive challenges: 1) maintenance of joint function and 2) potential for forearm growth. Autogenous vascularized proximal fibula free flap with physis for reconstruction of the pediatric distal radius is an important surgical technique which meets the aforementioned criteria. We describe the steps of our reconstruction and summarize the expected long-term outcomes based on the literature.

METHOD: A 9 year-old girl presented with a distal radius osteosarcoma extending up to the wrist joint. The plan was for an oncological resection of the distal radius and reconstruction utilizing a vascularized free proximal fibula flap. A two teams approach, orthopedic and plastic surgery, performed the procedure. Challenges observed included the tedious dissection of the proximal fibula underneath the common peroneal nerve and in establishing a retrograde venous flow after flap insetting.

A literature review using Ovid was performed and all articles with similar presentation are discussed including their methods and results.

CONCLUSIONS: When indicated, this procedure is safe with satisfactory range of motion of wrist and forearm growth observed at follow-ups in both the case presented and in the literature.

Learning Objective:

At the end of this presentation, participants will become familiar with:

- Requirement of maintaining joint function and growth potential in pediatric oncologic wrist reconstruction.
- Unique blood supply of the proximal fibula and its growth plate from the anterior tibial artery.

- Challenges associated with reverse free flap venous anastomosis when venous flow is retrograde.

- Difficulties of harvesting the proximal fibula while preserving all the branches of the common peroneal nerves.

- Long-term outcomes of the procedure based on the literature.

\section{7}

\section{PARTIAL TIBIAL NERVE TRANSFER TO THE TIBIALIS ANTERIOR MOTOR BRANCH FOR THE TREATMENT OF PERONEAL NERVE INJURY}

\section{J Giuffre, A Bishop, RJ Spinner, BA Levy, AY Shin}

PURPOSE: Traumatic peroneal nerve ruptures may preclude nerve grafting secondary to the length of nerve injury. The resultant foot drop is often treated with tendon transfers or AFO. Partial nerve transfers of an uninjured nerve to the motor branch of a denervated muscle are successful in the upper extremity.

The purpose is to evaluate patient outcomes following traumatic peroneal nerve ruptures treated with a partial nerve transfer from the tibial nerve to the motor branch of tibialis anterior.

METHODS: Patients treated with nerve transfers for foot drop at the Mayo Clinic, Rochester were reviewed. Patient demographics, mechanism of injury and time to surgery were recorded. Perioperative motor grading by the British Medical Research Council was obtained. Patients completed questionnaires regarding disability and satisfaction.

RESULTS: Eleven male patients underwent tibial nerve to deep peroneal nerve transfers an average 5.8 months postinjury. Four patients $(36 \%)$ had successful restoration of grade $3+$ ankledorsiflexion, one patient (9\%) regained grade 2, two patients $(18 \%)$ regained grade 1 and four patients (36\%) did not regain muscle activity. Clinically apparent motor recovery occurred ave. 7.6 months postoperatively. Reinnervation was demonstrated electromyographically ave. 9.1 months postoperatively.

Most patients (71\%) could ambulate and participate in activities. Although $71 \%$ of patients did not wear an AFO, only $29 \%$ of patients did not limp. The donor deficits included weak toe flexion $(29 \%)$ and reduced calf circumference (57\%).

Overall, $57 \%$ of patients were satisfied and $71 \%$ would recommend surgery.

CONCLUSION: Nerve transfers for foot drop give inconsistent results. Although 36\% of patients achieved M3 + recovery, the remaining patients regained minimal motion and risked the morbidity of the procedure.

Learning Objectives:

- The surgeon will appreciate the severity of peroneal nerve ruptures, the difficult reconstruction and the few available treatment options.

- The surgeon will acknowledge the inconsistent outcomes using nerve transfers for ankle-dorsiflexion between the literature and our study.

\section{8}

\section{TREATMENT OF SECONDARY LYMPHEDEMAS BY MICROSURGICAL LYMPH VESSEL TRANSPLANTATION:} A REVIEW OF 14 CASES

\section{Tobbia, G Felmerer}

BACKGROUND: Chronic lymphedema is a problematic complication of cancer diagnosis and therapy. It remains a poorly understood condition that has the potential to occur after any intervention affecting lymph node drainage mechanism. Microsurgical lymph vessel transplantation is increasingly recognized as a promising method for bypassing the disrupted lymph drainage pathways and inducing long-term decrease of swelling in the affected limb. The purpose of the present study is to report on our experience to alleviate secondary lymphedema including upper limb, lower limb, genital and facial edemas.

METHODS: A review of 14 patients with post-operative lymphedema treated with autologous lymph vessel transplantation between October 2005 and November 2009; the senior author operated all patients. Two 
to four lymphatic vessel grafts are harvested from the inner aspect of the thigh and used for bypassing areas of obstructed lymph flow. The grafts are interposed between ascending lymphatics in the affected limb and the lymphatics beyond the obstruction. In addition to limb circumference measurements at several locations, patients underwent pre- and post-operative magnetic resonance lymphangiography to visualize the lymphatic pathways.

RESULTS: Seven of the patients had unilateral upper limb edema, all showed a marked reduction of limb circumference as early as a few days following surgery, this ranged from 20\% to 60\% (average 39.8\%) decrease of lymphedema. This improvement continued steadily over a follow up period of 3 years. The benefit of lymph vessel transplantation in the five patients that had unilateral lower limb lymphedema was less pronounced than the upper limb, this ranged from 10\% to 33\% (average $20 \%)$. One patient received lymph vessel transplantation for isolated penis shaft lymphedema; he had striking resolution of edema. The patient with unilateral facial edema, showed diminished lymphedema and more symmetry of facial appearance.

CONCLUSION: Lymph vessel transplantation resulted in improved lymphatic drainage in patients with secondary lymphedema. We were able to demonstrate long-term patency of the lymph vessel anastomosis by magnetic resonance lymphangiography.

\section{P1}

\section{VENOUS MALFORMATION OF MEDIAN NERVE VASA NERVORUM}

M Choi, G Althubaiti, A Islur

BACKGROUND: We report the diagnosis and management of a condition not yet described in the literature: a venous malformation of the median nerve vasa nervorum.

METHODS: A healthy teenage female presented with a 3 year history of increasing pain and variable swelling to the hand and wrist. Subtle blue discolouration was observed on the volar wrist and ulnar aspect of the hand. MRI confirmed a venous malformation arising from an indeterminate superficial source in the wrist, palm, and ulnar two digits, extending to the superficial subcutaneous layer. Operative planning included the possibility of devitalizing or resecting skin and requiring skin coverage, in addition to possible resection and reconstruction of nerves, tendons, or vessels. Intra-operative exploration confirmed a venous malformation arising from vasa nervorum of the median nerve and a separate venous malformation of the venae comitantes of the ulnar artery and digital arteries to the ring and small fingers. Microscopeassisted intrafascicular nerve dissection facilitated complete nerve preservation and complete resection of the median nerve malformation of $8 \mathrm{~cm} \times 4 \mathrm{~cm}$ as well as near-complete resection of the ulnar venae comitante lesion. Remaining structures were spared and skin flaps were preserved.

RESULTS: All wounds healed well and the patient had complete resolution of pain and symptoms at 9 month follow up.

CONCLUSIONS: To the best of our knowledge, this is the first report of a venous malformation arising from the median nerve vasa nervorum. Intrafascicular dissection allowed near-complete resection of the lesion, resolution of symptoms, and restoration of function. We suggest obtaining MRI under tourniquet without exsanguinations so as to engorge and dilate the lesion for optimal visualization.

Learning Objective:

- Discuss the challenges of resecting and reconstructing venous malformations of vasa nervorum.

\section{P2}

\section{PHOSPHODIESTERASE III INHIBITOR - MILRINONE - EFFECTS ON ISCHEMIA-REPERFUSION INJURY AND POTENTIAL CLINICAL USE IN FREE FLAPS}

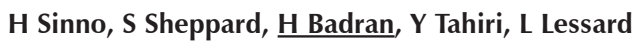

Free tissue transfers have been widely used across the globe for primary reconstruction following trauma, extensive resection of cancer and prompt resurfacing of mucosal or skin defects, restoration of bony supports, and reconstruction of specific organs. In free flap procedures, tissue is transferred from a body donor site with the aim of reconstructing a defect and obtaining a better functional and/or cosmetic result. The blood supply is disrupted and then a new blood supply is created at the recipient area. These flaps are vascularized by local vessels which are anastomosed to the flap by microvascular techniques. Different kinds of free flaps are in use and include: cutaneous flaps to reconstruct defects in the oral cavity, osseous flaps (e.g. fibula) to reconstruct mandibular defects, and free jejunal interposition to reconstruct the defect created by a total laryngopharyngectomy.

In consequence of the inflammatory reactions and injury resulting from processes such as ischemia-reperfusion (IR), intense research has been conducted with the aim of finding pharmacological agents to most suitably curtail these non-desirable reactions. Milrinone fits into one class of such agents. The actions of milrinone encompass that of the many pharmacologic agents described: anti-inflammatory, antiplatelet, anti-thrombotic, anti-leukocytic, anti-oxygen radicals, and anti-vasoconstrictive. It is a great agent in decreasing IR injury and thrombosis complications that may occur in free flap transfers. Milrinone's application to such procedures has never before been suggested but its potential for this is extraordinary.

The purpose of this review is to provide a framework for understanding the mechanisms of action of milrinone in the potential to enhance the viability of tissue transplantation particularly that of free flaps. Further clinical research must be conducted on milrinone and its effects on IR injury in free flap models.

\section{P3 \\ QUALITATIVE ASSESSMENT OF PATIENT EXPERIENCES FOLLOWING SACRECTOMY}

\section{K Davidge, C Eskicioglu, J Lipa, P Ferguson, C Shallow, F Wright}

PURPOSE: Complex pelvic resections involving sacrectomy are challenging to reconstruct and are associated with significant complication rates. Clinical outcomes in this patient population are not well understood. This study sought to examine the short and long-term challenges faced by patients undergoing sacrectomy, with the aim of improving patient education and experiences.

METHODS: Patients with pelvic neoplasms requiring partial or total sacrectomy were identified from a prospectively maintained database (1992-2007). The experiences, decision-making, quality of life, and supportive care needs of these patients were examined through semistructured interviews. Specific questions regarding pain, cosmesis, and functional challenges were addressed. Patient interviews were transcribed verbatim and analyzed using qualitative research methodology (grounded theory approach).

RESULTS: Twelve patients were interviewed ( 6 female, 32-82 years of age). The majority of patients had an S2-S3 sacrectomy with pedicled VRAM reconstruction. Median interview time was 32 minutes, and median follow-up was 78 months. Interview analysis revealed 5 broad themes: (1) the life-changing impact of surgery; (2) significant chronic pain; (3) satisfaction with immediate postoperative care; (4) need for additional long-term education and support; and (5) gratitude to be alive. Specific functional issues related to sacrectomy (e.g. difficulty sitting, pain) were of consistent and central importance to patients' quality of life. Other challenges (e.g. cosmesis) had variable individual significance.

CONCLUSIONS: Oncologic pelvic surgery necessitating sacrectomy and reconstruction is a life-changing event for patients and their families. These patients require additional education and support regarding the long-term sequelae of this procedure. Multidisciplinary involvement in both the immediate and late stages of patient recovery, such as through survivorship care plans, may be of significant benefit in this patient population.

Learning Objectives:

- To understand the patient experience of a sacrectomy and reconstruction.

- To identify gaps in the current level of patient education and propose strategies for improvement. 


\section{A}

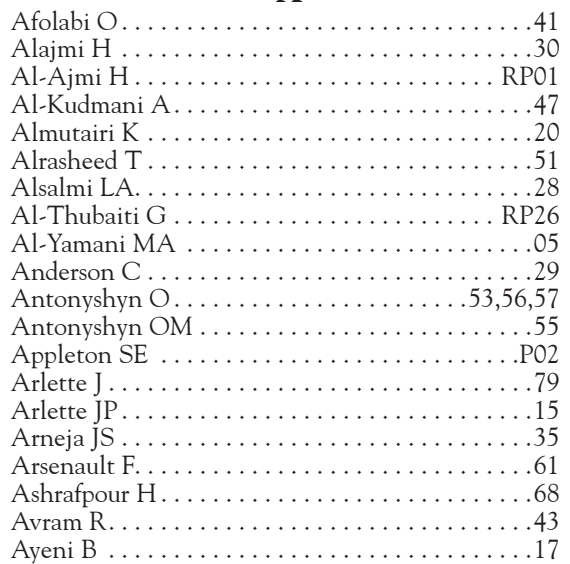

B

Bain J

Baltzer $\mathrm{H}$

Bebbington C.

Bellows D. .

Ben David G .

Benoit $\mathrm{P}$

Benson A . .

Bergeron L
Bezuhly M

Bhandari M

Binhammer P . .

Binhammer PA .

Blazer S.

Bobyn D. . .

Borsuk DE

Borsuk R.

Bortoluzzi P

Botros E

Bowman C.

Boyd K

Brown E

Brutus JP

Buchel EW

Bush K . .

Byers B .

\section{C}

Cahoon NJ

Calder K.

Campbell E. . .

Caouette-Laberge L

Card A.

Cartotto R

Catapano J...

Chan K. ...

Chan KM . .

Chan M

Chan S. . .

Chang PS. .

Chesney A . . .

Cheung K. .

Chivers Q

Chollet A

Chuback J. .

Chung B.

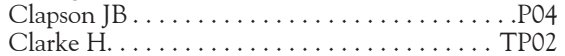

Coleman SR. . . . . . . . . . . Eye-Opener Session I,22

Cooper MJ

Courtemanche D ...

Courtemanche DJ

Cross KM . . .

Cugno $S$

Cullen R. .

Cypel TKS

Dal Cin A

Danino AM

Davidge K. . .

Davidson J

Davis A

Deheshi BM
Dempsey D.

Dickson L . . .

Ding J . .

Dirks P . .

Dumas A

Durlacher K

Edwards DC

Egerszegi EP . .

Elahi M

El-Khatib $\mathrm{H}$

Elzinga $\mathrm{K}$

Esmon CT

Farhangkhoee

Farrokhyar F.

Ferguson PC
Fialkov JA

Fialkov

Fish JS

Fisher D

Fisher DM .
Flannigan $\mathrm{R}$

Flannigan $\mathrm{R}$.

Forrest CR

Fouda Neel O.

Fouda-Neel O.

Frank SG ...

Fraulin F.

Friesen JR.

Gan L. . .

Gan LS.

Gaudreau G . . .

Ghazarian D. . .

Gieni M

Gilardino M ...

Gilardino MS

Gillardino M

Gjoska N

Gomez M

Gordon T

Graham B. .

Graham DA

Grenier-Vallée P

Griffin A

Guay N. .

Guyuron B

Haidar ZS. . .

Hall-Findlay EJ.

Hamdy RC.

Hamid KS .

Harris PG.

Harrop R

Hassa A ...

Haugrud M.

Hayakawa TEJ . .

Haykal S. . .

Hebert J . . .

Ho AL . .

Hofer SOP

Hontscharuk R.

Huang N.

Hynes S . . .

Islur A

Izadpanah A
Izadpanah A

Jada NG

Jansen L. . .

Jansen LA. .

Jean $M$.

Johnston B

Kanevsky J..

Kent B

Keswani S. .

Kim FS. . .

\section{E}

$\ldots \ldots \ldots \ldots \ldots 75$

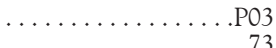

.73

08

02

.79

\section{$\mathrm{F}$}

.32

66

40

.05

6 L L

L L

L Lip

Lip

Lipa JE . . . . . . . . . . . . . . . . . . . . . . 444,68

Liwski RS $\ldots \ldots \ldots \ldots \ldots \ldots \ldots \ldots \ldots \ldots \ldots \ldots \ldots \ldots \ldots \ldots \ldots \ldots \ldots \ldots \ldots \ldots$
Loiselle FB $\ldots \ldots \ldots \ldots \ldots \ldots \ldots \ldots$

Luc M................................ P12

Lutfy J..............................

Lyons MV............................. RP27

Macadam S.......................42

Macadam SA .......................... 39

MacArthur I . . . . . . . . . . . . . . . RP22

Macarthur I . ....................... RP19

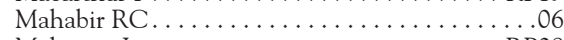

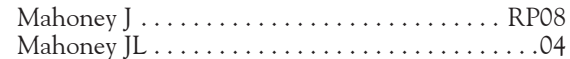

Mahoney $\mathrm{M}$...

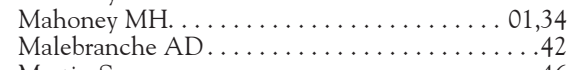

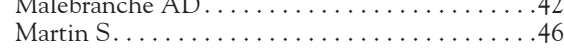

Matic DB ..................... RPO

McBeth P............................ RPO3

McDonald DS .............................. 27

McKenzie CD. ........................79

McKinnon JG . .......................... 79

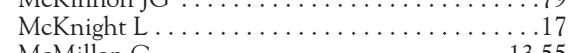

McMillan C.................... 13,55

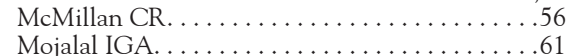

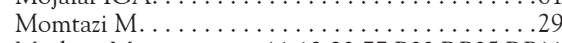

Morhart M . . . . . . . . 11,12,23,77,P03,RP05,RP11

Morris SF . . . . . . . . . . . . . 20,32,P02,RP09

Murabit A . . . . . . . . . . . . . . . . . . . . . 77

Murphy A $\ldots \ldots \ldots \ldots \ldots \ldots \ldots \ldots \ldots \ldots \ldots \ldots \ldots \ldots \ldots \ldots$
Murphy M $\ldots \ldots \ldots \ldots \ldots \ldots \ldots \ldots \ldots$

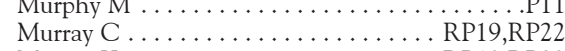

Murray K . . . . . . . . . . . . . . . . . . RP19,RP22

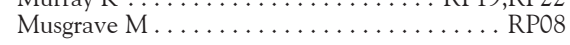

Neinstein R ......................... . 44

Neligan PC. . . . . . . . . . . . . . . . . . . . 444

Nelson RA . . . . . . . . . . . . . . . . . . . 64

$\underset{\mathrm{Ngan} A}{\mathrm{Ng} W \ldots \ldots \ldots \ldots \ldots \ldots \ldots \ldots \ldots} \mathrm{RP}_{\mathrm{PO} 2}$

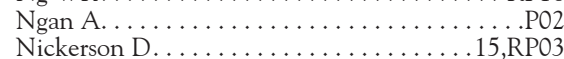

Nickerson $D \ldots \ldots \ldots \ldots \ldots \ldots \ldots \ldots \ldots \ldots \ldots \ldots \ldots \ldots \ldots \ldots \ldots$
Nikolis A $\ldots \ldots \ldots \ldots \ldots \ldots$

Nimigan AS . . . . . . . . . . . . . . . . . RP07

I $\ldots$. TP05,RP19,RP22

O'Grady K . . . . . . . . . . . . . . . . 77

Olson J . . . . . . . . . . . . 11,12,23,25,P03,RP11

Olson JL $\ldots \ldots \ldots \ldots \ldots \ldots \ldots \ldots \ldots \ldots \ldots \ldots \ldots \ldots$
Ouatik N $\ldots \ldots \ldots \ldots \ldots \ldots \ldots \ldots \ldots \ldots \ldots$

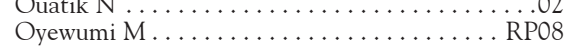

$\mathbf{P}$
Paek LS $\ldots \ldots \ldots \ldots \ldots \ldots \ldots \ldots \ldots$ RP01

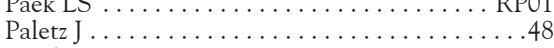

Pandya AN. . . . . . . . . . . . . . . . . . . 64

Pang C . . . . . . . . . . . . . . . . . . . . . . . . . .69

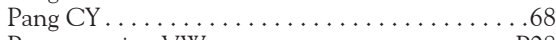

Papanastasiou VW $\ldots \ldots \ldots \ldots \ldots \ldots \ldots \ldots \ldots \ldots$
Papp A

Pappas K........................... 35 
Pavri S

Perkins A

Peters D

Philip A

Piggott J

Pilipchuk $\mathrm{C}$

Pirani A

Power $\mathrm{H}$

Power HA

Prochazka A

$\mathrm{R}$

Rai SS

$\ldots \ldots \ldots \ldots \ldots 60 . \cdots \ldots$

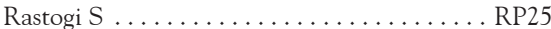

Ravid EN . . . . . . . . . . . . . . . . . . 11

Rechner B .................... . . . . . . . .

Rideout A. . . . . . . . . . . . . . . . . . 57

Rizis D . . . . . . . . . . . . . . . . 37,RP25

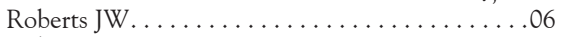

Robinson J . . . . . . . . . . . . . . . . . 21

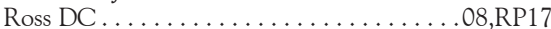

Ross L................. TP07,RP26

$\mathrm{S}$

Sampalis J. . . . . . . . . . . . . . . 19

Samson T. . . . . . . . . . . . . . 58,76

Sass $\mathrm{K} \ldots \ldots \ldots \ldots \ldots \ldots \ldots \ldots \ldots \ldots \ldots \ldots \ldots \ldots \ldots \ldots \ldots \ldots \ldots \ldots$
Sattler $\mathrm{T} \ldots \ldots \ldots \ldots \ldots$

Schachar N

Schwarz K

Scilley CG . . . . . . . . . . . . . . . . . TP08

Seal A.

Seal SKF. . .

.

Sharma N. . . . . . . . . . . . . . . RP10

Sharma V . . . . . . . . . . . . . . . . 78

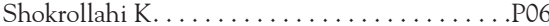

Sigurson L . . . . . . . . . . . . . . . . . 27

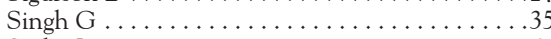

Sinha I . . . . . . . . . . . . . . . . . . . .64

Sinno H . . . 54,63,70,80,P08,P10,P12,RP01,RP15

Smith $\mathrm{S} \ldots \ldots \ldots \ldots \ldots \ldots \ldots \ldots \ldots \ldots$

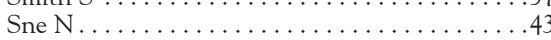

Song J. . . . . . . . . . . . . . . . . . . . . .06

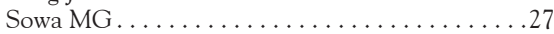

St. Amand $\mathrm{H} \ldots \ldots \ldots \ldots \ldots \ldots \ldots \ldots \ldots$. $\ldots \ldots \ldots$

Steinbok P .......................07

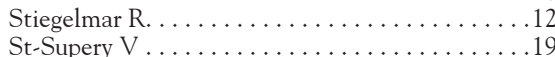

St-Supery $V \ldots \ldots \ldots \ldots$

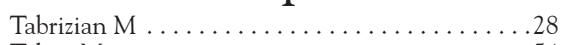

Tahiri M. . . . . . . . . . . . . . . . . . 54

Tahiri Y . . . . . . . . . 19,54,62,63,P12,RP15

Tang DT . . . . . . . . . . . . . .

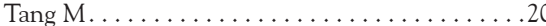

Tardif M . . . . . . . . . . . . . . . . . RP21

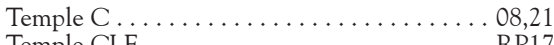

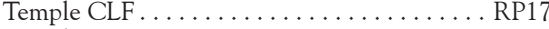

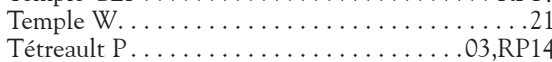

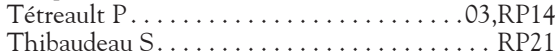

Thoma A . . . . . . . . . . . . 16,17,46,RP12,RP13,RP16

Tobbia D. . . . . . . . . . . . . P05,P07

Todd K

Tomlinson $\mathrm{G} \ldots \ldots \ldots \ldots \ldots \ldots \ldots \ldots \ldots \ldots \ldots$

Tredget E . . . . . . . . . . . . . . . . . . 25,29

Tremblay D. . . . . . . . . . . . . . . RP21

Tremblay DM. . . . . . . . . . . . . . . .03,RP14

Trzeciak A . . . . . . . . . . . . . . . . . . . . 30,P09

Tung A ..................................

Tyldesley S

$\mathrm{V}$

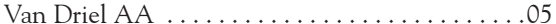

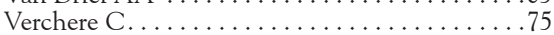

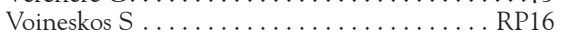

Vorstenbosch J . . . . . . . . . . . . . . . . . . . . . . 30,P09

W

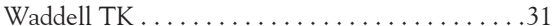

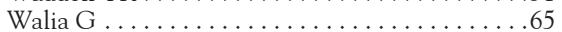

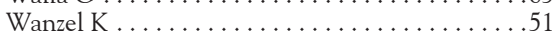

Watkins JF . . . . . . . . . . . . . . .64

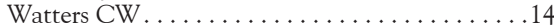

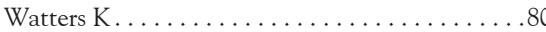

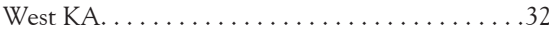

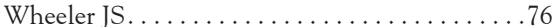

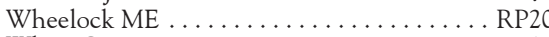

White C. . .

..16
White CP.

White IV RR

Williams BH

Williams J.

Williams JG

Williamson S

Winocour $S$

Wong $\mathrm{K}$

Wong $\mathrm{R}$

Wong $\mathrm{V}$

Wunder J ...

Wunder JS .

\section{$\mathrm{Y}$}

Yarascavitch A

Yau FM.

Yazdani A . .

Yeung JK. .

Zhong T

Zhu J. . .

Zhu LF.

\section{GAM AUTHOR INDEX}

A

Almutairi $\mathrm{K}$

Althubaiti $\mathrm{G}$

\section{B}

Badran H

Bartlett E

Bettger-Hahn M.

Bezuhly M

Bishop A

Bou-Merhi J .

Boyd B . . .

Boyd K . .

Brooks D.

Buchel E.

Buncke G.

Buncke GM

Buntic R.

Buntic RF.

Choi M

Chu M .

Cook EF.
Cook Jr EF

Cordeiro PG.

Davidge $K$

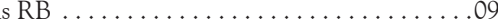

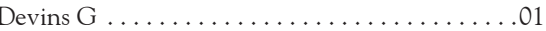

Disa JJ. . . .

Dissanayake D

E

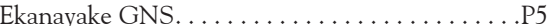

Eskicioglu C

Felmerer G

$\mathrm{F}$

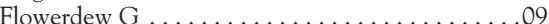

Fortin A . . .

Fouda neel $\mathrm{O}$

Gilbert R

$\ldots \ldots \ldots 01,02$

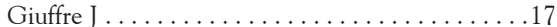

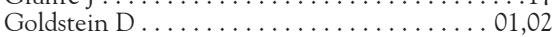

Greer-Bayramoglu R . . . . . . . . . . . . . . . . 07

Guay N. . . . . . . . . . . . . . . . . 11

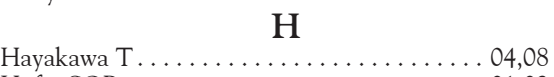

Hofer SOP . . . . . . . . . . . . . . . 01,02

Islur $\mathrm{A} \ldots \ldots \ldots \ldots \ldots$

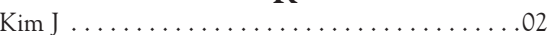

$\mathrm{L}$

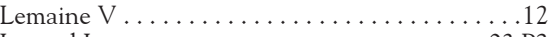

Lessard L. . . . . . . . . . . . . . . . . 03,P2

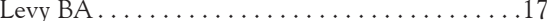

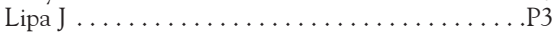

Logsetty S. . . . . . . . . . . . . . 04

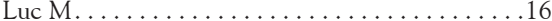

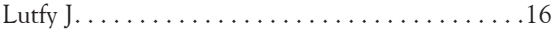

$\mathbf{M}$

MacDermid J . . . . . . . . . . . . . . . 13

McCarthy C. . . . . . . . . . . . . . . . 12

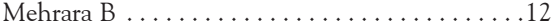

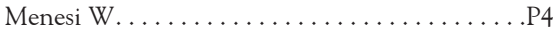

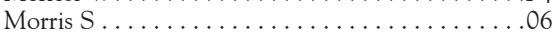

.

Perkins A........................ 03

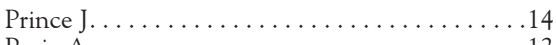

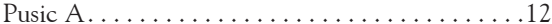

$\mathbf{R}$

Rasmussen P..............06

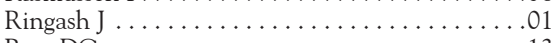

Ross DC . . . . . . . . . . . . . . 13

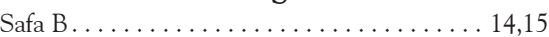

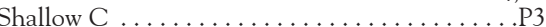

Sheppard S. . . . . . . . . . . . . .

Shin AY $\ldots \ldots \ldots \ldots \ldots \ldots \ldots \ldots \ldots \ldots \ldots \ldots \ldots$

Shokrollahi K.....................11

Sigurdson L . . . . . . . . . . . . . . . . 04,08

Sigurdson LJ . . . . . . . . . . . . . . . . . . . . .

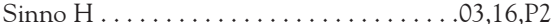

Spinner RJ . . . . . . . . . . . . . . 17

$\mathrm{T}$

Tahiri Y $\ldots \ldots \ldots \ldots \ldots \ldots \ldots \ldots \ldots \ldots \ldots$ P2

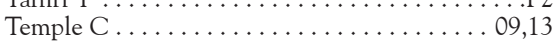

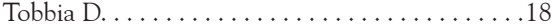

Williamson $S \ldots \ldots \ldots 5$

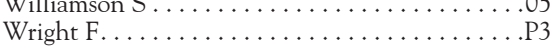

$\mathrm{Y}$

Yaghoubian AA . .................... 10

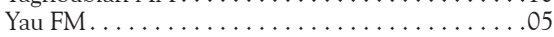

Yla-Kotola T . . . . . . . . . . . . . . . 02 


\section{P4}

EARLY EXPERIENCE WITH THE FIRST GROUP OF PERFORATOR FREE FLAPS IN A COMMUNITY HOSPITAL

\section{SETTING}

\section{W Menesi}

BACKGROUND: Perforator free flaps have become popular worldwide. Because of its perceived complexity and anticipated need of hospital resources, these flaps have traditionally been limited to the university hospital setting. Therefore, many community hospitals lack what may be considered the necessary equipment and monitoring skills and staff to perform successful microvascular surgery. Free flap monitoring has also become routine and reliable, with many surgeons simplifying this process for the nursing staff. We are reporting our early experience with the first group of perforator free flaps in our community hospital.

METHODS: Two patients underwent reconstruction using anterolateral thigh perforator free flaps. The first flap was used for lower extremity reconstruction in a 54 year old diabetic patient. The second flap was used for facial reconstruction in a 77 year old patient. Patients underwent immediate postoperative monitoring amounted to flap Doppler checks per our protocol and periodic monitoring of vital signs. In this group, a method of floor nurse monitoring was used to avoid the use and expense of the intensive care unit.

RESULTS: Two anterolateral perforator free flaps were successfully performed in our community hospital setting. Our hospital had no resident coverage; thus, we were solely dependent on our nurses' monitoring and evaluation. The nursing staff were initially not familiar with these free flaps in our community hospital. However, we found the nursing staff to be eager to participate in this level of patient care, with many requesting in-service presentations.

CONCLUSION: Our early experience has demonstrated that perforator free flaps can be safely and efficiently performed in a community hospital setting. This would then allow patient access to more contemporary reconstructions within the community.

Learning Objectives:

- At the end of this presentation the participant will learn about methods and challenges of performing successful perforator free flap in a community hospital setting. 